\title{
OPERATOR ERROR ESTIMATES IN THE HOMOGENIZATION PROBLEM FOR NONSTATIONARY PERIODIC EQUATIONS
}

\author{
M. SH. BIRMAN AND T. A. SUSLINA \\ To the memory of Tatyana Petrovna $I l^{\prime}$ ina
}

\begin{abstract}
Matrix periodic differential operators (DO's) $\mathcal{A}=\mathcal{A}(\mathbf{x}, \mathbf{D})$ in $L_{2}\left(\mathbb{R}^{d} ; \mathbb{C}^{n}\right)$ are considered. The operators are assumed to admit a factorization of the form $\mathcal{A}=\mathcal{X}^{*} \mathcal{X}$, where $\mathcal{X}$ is a homogeneous first order DO. Let $\mathcal{A}_{\varepsilon}=\mathcal{A}\left(\varepsilon^{-1} \mathbf{x}, \mathbf{D}\right), \varepsilon>0$. The behavior of the solutions $\mathbf{u}_{\varepsilon}(\mathbf{x}, \tau)$ of the Cauchy problem for the Schrödinger equation $i \partial_{\tau} \mathbf{u}_{\varepsilon}=\mathcal{A}_{\varepsilon} \mathbf{u}_{\varepsilon}$, and also the behavior of those for the hyperbolic equation $\partial_{\tau}^{2} \mathbf{u}_{\varepsilon}=-\mathcal{A}_{\varepsilon} \mathbf{u}_{\varepsilon}$, is studied as $\varepsilon \rightarrow 0$. Let $\mathbf{u}_{0}$ be the solution of the corresponding homogenized problem. Estimates of order $\varepsilon$ are obtained for the $L_{2}\left(\mathbb{R}^{d} ; \mathbb{C}^{n}\right)$-norm of the difference $\mathbf{u}_{\varepsilon}-\mathbf{u}_{0}$ for a fixed $\tau \in \mathbb{R}$. The estimates are uniform with respect to the norm of initial data in the Sobolev space $H^{s}\left(\mathbb{R}^{d} ; \mathbb{C}^{n}\right)$, where $s=3$ in the case of the Schrödinger equation and $s=2$ in the case of the hyperbolic equation. The dependence of the constants in estimates on the time $\tau$ is traced, which makes it possible to obtain qualified error estimates for small $\varepsilon$ and large $|\tau|=O\left(\varepsilon^{-\alpha}\right)$ with appropriate $\alpha<1$.
\end{abstract}

\section{$\S 0$. INTRODUCTION}

0.1. The class of operators. The present paper is a continuation of the authors'

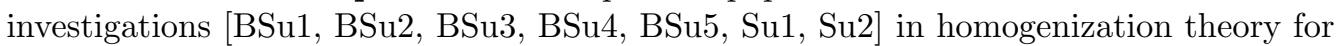
a class of (matrix) periodic differential operators (DO's) acting in the space $L_{2}\left(\mathbb{R}^{d} ; \mathbb{C}^{n}\right)$. This class is rather wide and includes many operators of mathematical physics.

We consider matrix elliptic positive second order DO's in $L_{2}\left(\mathbb{R}^{d} ; \mathbb{C}^{n}\right)$ that admit a factorization of the form

$$
\mathcal{A}=\mathcal{A}(g, f)=f(\mathbf{x})^{*} b(\mathbf{D})^{*} g(\mathbf{x}) b(\mathbf{D}) f(\mathbf{x}) .
$$

Here $b(\mathbf{D})$ is a homogeneous matrix first order DO with constant coefficients. Its symbol $b(\boldsymbol{\xi})$ is an $(m \times n)$-matrix of rank $n$ (we assume that $m \geq n)$. It is assumed that the matrix-valued functions $f(\mathbf{x})$ (of size $n \times n)$ and $g(\mathbf{x})$ (of size $m \times m$ ) are periodic with respect to some lattice $\Gamma$ in $\mathbb{R}^{d}$ and that

$$
g(\mathbf{x})>0 ; \quad g, g^{-1} \in L_{\infty} ; \quad f, f^{-1} \in L_{\infty} .
$$

For a more precise description of the operators (0.1), see Subsection 4.1.

It is convenient to start with a narrower class of operators of the form

$$
\widehat{\mathcal{A}}=\widehat{\mathcal{A}}(g)=b(\mathbf{D})^{*} g(\mathbf{x}) b(\mathbf{D}),
$$

and accordingly, to accept the "two-level" order of exposition.

2000 Mathematics Subject Classification. Primary 35B27.

Key words and phrases. Periodic operators, nonstationary equations, Cauchy problem, threshold effect, homogenization, effective operator.

Supported by RFBR (grant no. 08-01-00209-a) and "Scientific Schools" grant no. 816.2008.1.

(c)2009 American Mathematical Society 
The operators $(0.1)$ and $(0.2)$ are viewed as selfadjoint operators in $L_{2}\left(\mathbb{R}^{d} ; \mathbb{C}^{n}\right)$. The bottom of the spectrum of these operators is the point $\lambda_{0}=0$.

Now we introduce a small parameter $\varepsilon>0$ and denote $\varphi^{\varepsilon}(\mathbf{x}):=\varphi\left(\varepsilon^{-1} \mathbf{x}\right)$ for any $\Gamma$-periodic function $\varphi(\mathbf{x})$. Consider the operators

$$
\begin{aligned}
& \mathcal{A}_{\varepsilon}=\left(f^{\varepsilon}(\mathbf{x})\right)^{*} b(\mathbf{D})^{*} g^{\varepsilon}(\mathbf{x}) b(\mathbf{D}) f^{\varepsilon}(\mathbf{x}), \\
& \widehat{\mathcal{A}}_{\varepsilon}=b(\mathbf{D})^{*} g^{\varepsilon}(\mathbf{x}) b(\mathbf{D}),
\end{aligned}
$$

whose coefficients oscillate rapidly as $\varepsilon \rightarrow 0$.

0.2. Homogenization for elliptic and parabolic problems. The homogenization problem for elliptic equations in $\mathbb{R}^{d}$ can be regarded as a problem of asymptotic description of the resolvent $\left(\mathcal{A}_{\varepsilon}+I\right)^{-1}$ as $\varepsilon \rightarrow 0$. For definiteness, here we shall talk about the simpler operators (0.4). In classical homogenization theory, the following fact is crucial: there exists an effective operator $\widehat{\mathcal{A}}^{0}=b(\mathbf{D})^{*} g^{0} b(\mathbf{D})$ with constant effective matrix $g^{0}$ such that the resolvent $\left(\widehat{\mathcal{A}}_{\varepsilon}+I\right)^{-1}$ tends (in some sense) to the resolvent $\left(\widehat{\mathcal{A}}^{0}+I\right)^{-1}$ as $\varepsilon \rightarrow 0$. Usually, strong (or even weak) convergence is considered. Further correction terms are constructed, the main one of which is the term $\varepsilon K(\varepsilon)$ of order $\varepsilon$, where the operator $K(\varepsilon)$ is called a corrector. Usually, remainder estimates are found under some smoothness conditions on the coefficients and on the right-hand sides of the corresponding equations.

The rule of constructing the effective matrix $g^{0}$ is well known. Under our assumptions, this rule is given in $\S 6$ below.

In the papers BSu1, BSu2, for the first time in homogenization theory, it was shown that the resolvent $\left(\widehat{\mathcal{A}}_{\varepsilon}+I\right)^{-1}$ tends to the resolvent $\left(\widehat{\mathcal{A}}^{0}+I\right)^{-1}$ in the operator $L_{2}$-norm as $\varepsilon \rightarrow 0$. The following estimate is true:

$$
\left\|\left(\widehat{\mathcal{A}}_{\varepsilon}+I\right)^{-1}-\left(\widehat{\mathcal{A}}^{0}+I\right)^{-1}\right\|_{L_{2}\left(\mathbb{R}^{d}\right) \rightarrow L_{2}\left(\mathbb{R}^{d}\right)} \leq C \varepsilon .
$$

The constant $C$ depends only on the parameters of the lattice $\Gamma$ and on the upper and lower bounds for the matrix-valued function $g(\mathbf{x})$ and for the symbol $b(\boldsymbol{\theta})^{*} b(\boldsymbol{\theta})$ with $|\boldsymbol{\theta}|=1$.

In $[\mathrm{BSu} 3, \mathrm{BSu} 4$, a more accurate approximation for the resolvent of the operator (0.4) was found:

$$
\left\|\left(\widehat{\mathcal{A}}_{\varepsilon}+I\right)^{-1}-\left(\widehat{\mathcal{A}}^{0}+I\right)^{-1}-\varepsilon K(\varepsilon)\right\|_{L_{2}\left(\mathbb{R}^{d}\right) \rightarrow L_{2}\left(\mathbb{R}^{d}\right)} \leq C \varepsilon^{2} .
$$

In this approximation, the corrector was taken into account. In BSu5, the resolvent of the operator (0.4) was approximated in the norm of the operators acting from $L_{2}\left(\mathbb{R}^{d} ; \mathbb{C}^{n}\right)$ to the Sobolev space $H^{1}\left(\mathbb{R}^{d} ; \mathbb{C}^{n}\right)$ :

$$
\left\|\left(\widehat{\mathcal{A}}_{\varepsilon}+I\right)^{-1}-\left(\widehat{\mathcal{A}}^{0}+I\right)^{-1}-\varepsilon K_{1}(\varepsilon)\right\|_{L_{2}\left(\mathbb{R}^{d}\right) \rightarrow H^{1}\left(\mathbb{R}^{d}\right)} \leq C \varepsilon .
$$

Herewith, $K_{1}(\varepsilon)$ is the traditional corrector used in homogenization theory, while the corrector $K(\varepsilon)$ in (0.6) is the sum of three terms $K(\varepsilon)=K_{1}(\varepsilon)+K_{1}(\varepsilon)^{*}+K_{3}$, where $K_{3}$ does not depend on $\varepsilon$. Note that, in estimate $(0.7)$, the corrector $K_{1}(\varepsilon)$ can be replaced by the more complicated corrector $K(\varepsilon)$, which will influence the estimational constant only. At the same time, in (0.6) it is impossible to replace $K(\varepsilon)$ by $K_{1}(\varepsilon)$. In [BSu2, BSu3, BSu4, BSu5], similar but somewhat more complicated approximations were obtained for the resolvent of the operator $(0.3)$.

Along with results for the general class of operators (0.1), (0.2), the authors' approach was also applied to homogenization of the stationary periodic Maxwell system. This problem was studied in [BSu2, Chapter 7] and in [BSu6, Su3, Su4, Su5. The study of the Maxwell operator required an essential development of the techniques. 
In [Su1, Su2], the approach suggested in [BSu1, BSu2, BSu3, BSu4, BSu5] was applied also to homogenization of the Cauchy problem for a parabolic equation (the short communication [Su1] contained a detailed description of the results, and the full exposition was given in [Su2]). The problem is reduced to the study of the operator exponential $\exp \left(-\widehat{\mathcal{A}}_{\varepsilon} \tau\right), \tau>0$. In [Su1, Su2], the following approximation was obtained:

$$
\left\|\exp \left(-\widehat{\mathcal{A}}_{\varepsilon} \tau\right)-\exp \left(-\widehat{\mathcal{A}}^{0} \tau\right)\right\|_{L_{2}\left(\mathbb{R}^{d}\right) \rightarrow L_{2}\left(\mathbb{R}^{d}\right)} \leq C \varepsilon \tau^{-1 / 2} .
$$

In the paper $[\mathrm{V}]$ by E. S. Vasilevskaya, a more accurate approximation with a corrector was found:

$$
\left\|\exp \left(-\widehat{\mathcal{A}}_{\varepsilon} \tau\right)-\exp \left(-\widehat{\mathcal{A}}^{0} \tau\right)-\varepsilon \mathcal{K}(\varepsilon, \tau)\right\|_{L_{2}\left(\mathbb{R}^{d}\right) \rightarrow L_{2}\left(\mathbb{R}^{d}\right)} \leq C \varepsilon^{2} \tau^{-1} .
$$

In [Su1, Su2, V], similar (but more complicated) approximations were obtained for the exponential $\exp \left(-\mathcal{A}_{\varepsilon} \tau\right)$ of the operator $(0.3)$.

0.3. The method of investigation. The method that allows us to obtain estimates of the form (0.5)-(0.9) can be called the spectral method. We mean that methods of spectral theory of selfadjoint operators in Hilbert space are used actively. Herewith, a significant part of the constructions is done in the framework of abstract operator theory, and only after that is specified to periodic differential operators. It turns out that the homogenization phenomenon has a threshold nature. This means that, for constructing the homogenized problem and estimating the error terms, only spectral characteristics of a periodic operator near the bottom of the spectrum (the so-called threshold characteristics) are essential. Also, methods of analytic perturbation theory can be used widely.

Note that the papers BSu1, BSu2 stimulated interest in operator norm error estimates in homogenization theory. In [Zh, Pas, ZhPas1, estimates of the form (0.5), (0.7) for the acoustics operator and for the operator of elasticity theory (which are of the form (0.4)) were deduced by a different (nonspectral) method. In [ZhPas2], a similar method was applied to obtain estimate $(0.8)$ for the acoustics operator $\widehat{\mathcal{A}}_{\varepsilon}=-\operatorname{div} g^{\varepsilon}(\mathbf{x}) \nabla$.

0.4. Nonstationary problems. So, in the case of elliptic and parabolic problems, the spectral approach to homogenization is developed in detail. The situation with homogenization of nonstationary Schrödinger-type and hyperbolic equations is different. The present paper is devoted to these problems. Herewith, we need to overcome new technical difficulties.

We consider the following Cauchy problem for the Schrödinger type equation with the operator (0.4):

$$
i \frac{\partial \mathbf{u}_{\varepsilon}(\mathbf{x}, \tau)}{\partial \tau}=\widehat{\mathcal{A}}_{\varepsilon} \mathbf{u}_{\varepsilon}(\mathbf{x}, \tau), \quad \mathbf{x} \in \mathbb{R}^{d}, \quad \tau \in \mathbb{R}, \quad \mathbf{u}_{\varepsilon}(\mathbf{x}, 0)=\phi(\mathbf{x}),
$$

where $\phi \in L_{2}\left(\mathbb{R}^{d} ; \mathbb{C}^{n}\right)$ is a given function.

Also, we consider the following Cauchy problem for the hyperbolic equation with the operator (0.4):

$$
\begin{aligned}
\frac{\partial^{2} \mathbf{v}_{\varepsilon}(\mathbf{x}, \tau)}{\partial \tau^{2}} & =-\widehat{\mathcal{A}}_{\varepsilon} \mathbf{v}_{\varepsilon}(\mathbf{x}, \tau), \quad \mathbf{x} \in \mathbb{R}^{d}, \quad \tau \in \mathbb{R}, \\
\mathbf{v}_{\varepsilon}(\mathbf{x}, 0) & =\boldsymbol{\phi}(\mathbf{x}), \quad \frac{\partial \mathbf{v}_{\varepsilon}}{\partial \tau}(\mathbf{x}, 0)=\boldsymbol{\psi}(\mathbf{x}),
\end{aligned}
$$

where $\boldsymbol{\phi}, \boldsymbol{\psi} \in L_{2}\left(\mathbb{R}^{d} ; \mathbb{C}^{n}\right)$ are given functions.

The corresponding "homogenized" problem for (0.10) has the form

$$
i \frac{\partial \mathbf{u}_{0}(\mathbf{x}, \tau)}{\partial \tau}=\widehat{\mathcal{A}}^{0} \mathbf{u}_{0}(\mathbf{x}, \tau), \quad \mathbf{u}_{0}(\mathbf{x}, 0)=\phi(\mathbf{x}),
$$


and the "homogenized" problem for problem (0.11) has the form

$$
\frac{\partial^{2} \mathbf{v}_{0}(\mathbf{x}, \tau)}{\partial \tau^{2}}=-\widehat{\mathcal{A}}^{0} \mathbf{v}_{0}(\mathbf{x}, \tau), \quad \mathbf{v}_{0}(\mathbf{x}, 0)=\phi(\mathbf{x}), \quad \frac{\partial \mathbf{v}_{0}}{\partial \tau}(\mathbf{x}, 0)=\boldsymbol{\psi}(\mathbf{x}) .
$$

Here $\widehat{\mathcal{A}}^{0}=b(\mathbf{D})^{*} g^{0} b(\mathbf{D})$ is the effective operator.

It turns out that, as $\varepsilon \rightarrow 0$, the solution $\mathbf{u}_{\varepsilon}$ of problem (0.10) tends to the solution $\mathbf{u}_{0}$ of the homogenized problem $(0.12)$ in $L_{2}\left(\mathbb{R}^{d} ; \mathbb{C}^{n}\right)$ for a fixed $\tau \in \mathbb{R}$. Similarly, the solution $\mathbf{v}_{\varepsilon}$ of problem (0.11) tends to the solution $\mathbf{v}_{0}$ of the homogenized problem (0.13).

0.5. Main results. Our main goal in the present paper is to obtain qualified estimates for $\mathbf{u}_{\varepsilon}-\mathbf{u}_{0}$ and $\mathbf{v}_{\varepsilon}-\mathbf{v}_{0}$ in the $L_{2}\left(\mathbb{R}^{d} ; \mathbb{C}^{n}\right.$ )-norm (for a fixed $\tau \in \mathbb{R}$ ) that are uniform with respect to the norms of initial data in an appropriate Sobolev space $H^{s}\left(\mathbb{R}^{d} ; \mathbb{C}^{n}\right)$.

We obtain the following result for the solutions of (0.10) (see Theorem 14.1). If $\phi \in H^{s}\left(\mathbb{R}^{d} ; \mathbb{C}^{n}\right)$ with some $0<s \leq 3$, then, as $\varepsilon \rightarrow 0, \mathbf{u}_{\varepsilon}$ tends to $\mathbf{u}_{0}$ in the $L_{2}\left(\mathbb{R}^{d} ; \mathbb{C}^{n}\right)$ norm for any fixed $\tau \in \mathbb{R}$. We have

$$
\left\|\mathbf{u}_{\varepsilon}(\cdot, \tau)-\mathbf{u}_{0}(\cdot, \tau)\right\|_{L_{2}\left(\mathbb{R}^{d}\right)} \leq \varepsilon^{s / 3} \mathcal{C}_{s}(\tau)\|\phi\|_{H^{s}\left(\mathbb{R}^{d}\right)} .
$$

The constant $\mathcal{C}_{s}(\tau)$ is $O\left(|\tau|^{s / 3}\right)$ for large $|\tau|$. This allows us to consider large values of time $|\tau|=\varepsilon^{-\alpha}$, where $0<\alpha<1$, and to obtain the estimate

$$
\left\|\mathbf{u}_{\varepsilon}\left(\cdot, \pm \varepsilon^{-\alpha}\right)-\mathbf{u}_{0}\left(\cdot, \pm \varepsilon^{-\alpha}\right)\right\|_{L_{2}\left(\mathbb{R}^{d}\right)} \leq \varepsilon^{s(1-\alpha) / 3} \mathcal{C}_{s}(1)\|\phi\|_{H^{s}\left(\mathbb{R}^{d}\right)} .
$$

If it is only known that $\phi \in L_{2}\left(\mathbb{R}^{d} ; \mathbb{C}^{n}\right)$, then we have convergence of $\mathbf{u}_{\varepsilon}$ to $\mathbf{u}_{0}$ in $L_{2}\left(\mathbb{R}^{d} ; \mathbb{C}^{n}\right)$ for $\tau \in \mathbb{R}$ fixed, and also convergence of the difference $\mathbf{u}_{\varepsilon}\left(\cdot, \pm \varepsilon^{-\alpha}\right)$ $\mathbf{u}_{0}\left(\cdot, \pm \varepsilon^{-\alpha}\right)$ (where $0<\alpha<1$ ) to zero in $L_{2}\left(\mathbb{R}^{d} ; \mathbb{C}^{n}\right)$.

The next result concerns the solutions of problem (0.11) (Theorem 15.1). If $\boldsymbol{\phi}, \boldsymbol{\psi} \in$ $H^{s}\left(\mathbb{R}^{d} ; \mathbb{C}^{n}\right)$ with some $0<s \leq 2$, then, as $\varepsilon \rightarrow 0, \mathbf{v}_{\varepsilon}$ tends to $\mathbf{v}_{0}$ in the $L_{2}\left(\mathbb{R}^{d} ; \mathbb{C}^{n}\right)$-norm for $\tau \in \mathbb{R}$ fixed. We have

$$
\left\|\mathbf{v}_{\varepsilon}(\cdot, \tau)-\mathbf{v}_{0}(\cdot, \tau)\right\|_{L_{2}\left(\mathbb{R}^{d}\right)} \leq \varepsilon^{s / 2}\left(\mathfrak{C}_{s}(\tau)\|\phi\|_{H^{s}\left(\mathbb{R}^{d}\right)}+\mathfrak{C}_{s}^{\prime}(\tau)\|\boldsymbol{\psi}\|_{H^{s}\left(\mathbb{R}^{d}\right)}\right) .
$$

The constant $\mathfrak{C}_{s}(\tau)$ is $O\left(|\tau|^{s / 2}\right)$, and $\mathfrak{C}_{s}^{\prime}(\tau)$ is $O\left(|\tau|^{1+s / 2}\right)$ for large $|\tau|$. This allows us to consider large values of time $|\tau|=\varepsilon^{-\alpha}$ and to obtain the estimate

$$
\begin{aligned}
\| \mathbf{v}_{\varepsilon}\left(\cdot, \pm \varepsilon^{-\alpha}\right) & -\mathbf{v}_{0}\left(\cdot, \pm \varepsilon^{-\alpha}\right) \|_{L_{2}\left(\mathbb{R}^{d}\right)} \\
& \leq \varepsilon^{s(1-\alpha) / 2}\left(\mathfrak{C}_{s}(1)\|\boldsymbol{\phi}\|_{H^{s}\left(\mathbb{R}^{d}\right)}+\varepsilon^{-\alpha} \mathfrak{C}_{s}^{\prime}(1)\|\boldsymbol{\psi}\|_{H^{s}\left(\mathbb{R}^{d}\right)}\right) .
\end{aligned}
$$

Here $0<\alpha<\frac{s}{s+2}$ if $\boldsymbol{\psi} \neq 0$, and $0<\alpha<1$ if $\boldsymbol{\psi}=0$.

If it is only known that $\boldsymbol{\phi}, \boldsymbol{\psi} \in L_{2}\left(\mathbb{R}^{d} ; \mathbb{C}^{n}\right)$, then we have strong convergence of $\mathbf{v}_{\varepsilon}$ to $\mathbf{v}_{0}$ in $L_{2}\left(\mathbb{R}^{d} ; \mathbb{C}^{n}\right)$ for $\tau \in \mathbb{R}$ fixed; in the case where $\boldsymbol{\psi}=0$, the difference $\mathbf{v}_{\varepsilon}\left(\cdot, \pm \varepsilon^{-\alpha}\right)-$ $\mathbf{v}_{0}\left(\cdot, \pm \varepsilon^{-\alpha}\right)$ (where $0<\alpha<1$ ) tends to zero in $L_{2}\left(\mathbb{R}^{d} ; \mathbb{C}^{n}\right)$.

The nonhomogeneous Cauchy problems (with nontrivial right-hand sides in the equations) are also studied. The corresponding results are contained in Theorems 14.2 and 15.2 .

Also, we get similar results for more general Cauchy problems with operators $(0.3)$ (see Theorems 14.3-14.6, 15.3-15.6).

In order to obtain the results described above, we study the behavior of the operator exponential $\exp \left(-i \mathcal{A}_{\varepsilon} \tau\right)$ (for the operators $(0.3)$ and $\left.(0.4)\right)$ for small $\varepsilon$. The solution of the Cauchy problem for the Schrödinger-type equation is given in terms of this exponential. In the case of the hyperbolic equation, we need to study the behavior of the operator $\cos \left(\tau \mathcal{A}_{\varepsilon}^{1 / 2}\right)$ for small $\varepsilon$.

We have not succeeded in finding full analogs of estimates (0.5) and (0.8). However, the spectral method allows us to obtain the following crucial facts. (As above, here we restrict ourselves to the case of the operators (0.4).) In order to obtain an estimate of order 
$\varepsilon$, we need to "smooth out" the operator-valued functions of $\widehat{\mathcal{A}}_{\varepsilon}$ under consideration, multiplying them by an appropriate power of the resolvent of the operator $\mathcal{H}_{0}=-\Delta$. Namely, we consider the operators $\exp \left(-i \tau \widehat{\mathcal{A}}_{\varepsilon}\right)\left(\mathcal{H}_{0}+I\right)^{-3 / 2}$ and $\cos \left(\tau \widehat{\mathcal{A}}_{\varepsilon}^{1 / 2}\right)\left(\mathcal{H}_{0}+I\right)^{-1}$ and obtain the following approximations in the operator norm in $L_{2}\left(\mathbb{R}^{d} ; \mathbb{C}^{n}\right)$ for them:

$$
\begin{aligned}
& \left\|\left(\exp \left(-i \tau \widehat{\mathcal{A}}_{\varepsilon}\right)-\exp \left(-i \tau \widehat{\mathcal{A}}^{0}\right)\right)\left(\mathcal{H}_{0}+I\right)^{-3 / 2}\right\|_{L_{2}\left(\mathbb{R}^{d}\right) \rightarrow L_{2}\left(\mathbb{R}^{d}\right)} \leq\left(C_{1}+C_{2}|\tau|\right) \varepsilon \\
& \left\|\left(\cos \left(\tau \widehat{\mathcal{A}}_{\varepsilon}^{1 / 2}\right)-\cos \left(\tau\left(\widehat{\mathcal{A}}^{0}\right)^{1 / 2}\right)\right)\left(\mathcal{H}_{0}+I\right)^{-1}\right\|_{L_{2}\left(\mathbb{R}^{d}\right) \rightarrow L_{2}\left(\mathbb{R}^{d}\right)} \leq\left(C_{3}+C_{4}|\tau|\right) \varepsilon
\end{aligned}
$$

Estimates (0.18) and (0.19) are appropriate analogs of estimates $(0.5),(0.8)$ and represent the main results of the paper in the operator terms (for the operators $(0.4)$ ). Estimate (0.14) follows from (0.18), and estimate (0.16) is deduced from (0.19).

Similar (but somewhat more complicated) approximations are obtained for the case of the operators $(0.3)$.

0.6. Method of investigation. By a scaling transformation, we can deduce (0.18) and (0.19) from the following two estimates (respectively):

$$
\begin{array}{r}
\left\|\left(\exp \left(-i \tau \varepsilon^{-2} \widehat{\mathcal{A}}\right)-\exp \left(-i \tau \varepsilon^{-2} \widehat{\mathcal{A}}^{0}\right)\right) \varepsilon^{3}\left(\mathcal{H}_{0}+\varepsilon^{2} I\right)^{-3 / 2}\right\|_{L_{2}\left(\mathbb{R}^{d}\right) \rightarrow L_{2}\left(\mathbb{R}^{d}\right)} \\
\leq\left(C_{1}+C_{2}|\tau|\right) \varepsilon, \\
\begin{aligned}
\|\left(\cos \left(\tau \varepsilon^{-1} \widehat{\mathcal{A}}^{1 / 2}\right)-\cos \left(\tau \varepsilon^{-1}\left(\widehat{\mathcal{A}}^{0}\right)^{1 / 2}\right)\right) \varepsilon^{2}\left(\mathcal{H}_{0}+\varepsilon^{2} I\right)^{-1} & \|_{L_{2}\left(\mathbb{R}^{d}\right) \rightarrow L_{2}\left(\mathbb{R}^{d}\right)} \\
& \leq\left(C_{3}+C_{4}|\tau|\right) \varepsilon .
\end{aligned}
\end{array}
$$

In order to obtain (0.20) and (0.21), we apply the unitary Gelfand transformation (see Subsection 4.3 below) and expand the operator $\widehat{\mathcal{A}}$ in the direct integral of operators $\widehat{\mathcal{A}}(\mathbf{k})$. The operators $\widehat{\mathcal{A}}(\mathbf{k})$ act in the space $L_{2}\left(\Omega ; \mathbb{C}^{n}\right)$ (where $\Omega$ is the cell of the lattice $\Gamma$ ) and depend on the parameter $\mathbf{k} \in \mathbb{R}^{d}$ (the quasimomentum). The operator $\widehat{\mathcal{A}}(\mathbf{k})$ is given by the differential expression $b(\mathbf{D}+\mathbf{k})^{*} g(\mathbf{x}) b(\mathbf{D}+\mathbf{k})$ with periodic boundary conditions; the spectrum of $\widehat{\mathcal{A}}(\mathbf{k})$ is discrete. The operator family $\widehat{\mathcal{A}}(\mathbf{k})$ is studied by methods of analytic perturbation theory (with respect to the one-dimensional parameter $t=|\mathbf{k}|$ ). We prove analogs of inequalities $(0.20)$ and $(0.21)$ for the operators $\widehat{\mathcal{A}}(\mathbf{k})$ with constants independent of $\mathbf{k}$. Then the inverse Gelfand transformation leads to (0.20) and (0.21).

We put $\mathbf{k}=t \boldsymbol{\theta}$, where $t=|\mathbf{k}|,|\boldsymbol{\theta}|=1$. To a great extent, the operator family $\widehat{\mathcal{A}}(\mathbf{k})=: \widehat{A}(t, \boldsymbol{\theta})$ can be treated within an abstract operator-theoretic framework. We study an operator family of the form $A(t)=X(t)^{*} X(t)$ in the Hilbert space $\mathfrak{H}$, where $X(t)=X_{0}+t X_{1}$. Suppose that the point $\lambda_{0}=0$ is an isolated eigenvalue of finite multiplicity for $A(0)$. We distinguish a finite rank operator $S$ (the so-called spectral germ of the operator family $A(t))$ acting in the space $\mathfrak{N}=\operatorname{Ker} A(0)$. The spectral germ (see Subsection 1.3 below) contains information about the threshold characteristics of the operator. In terms of the spectral germ, we find appropriate approximations for the operator-valued functions $\exp \left(-i \tau \varepsilon^{-2} A(t)\right)$ and $\cos \left(\tau \varepsilon^{-1} A(t)^{1 / 2}\right)$ multiplied by the corresponding "smoothing" factors. An application of these abstract results leads to the required estimates for differential operators.

In abstract considerations, we lean upon the threshold approximations for the operator family $A(t)$ obtained in $\mathrm{BSu} 1, \mathrm{BSu} 2$ and $\mathrm{Su} 2]$.

Note that the scaling transformation points to the threshold origin of the homogenization effect also for the nonstationary problems under consideration.

Essentially, our constructions are multistage. In the present paper, in order to clarify what new difficulties arise in the nonstationary problems under study, we restrict ourselves to first level questions. In particular, approximations and remainder estimates 
with corrector are left aside. Next, we did not touch upon the nonstationary Maxwell system, which does not satisfy the conditions of our general theorems. The study of this system requires separate considerations. We hope to turn to these problems elsewhere.

0.7. Now we dwell on earlier results concerning homogenization problems for the Schrödinger-type equation and the hyperbolic equations. Our survey does not claim to be exhaustive.

In $\left[\mathrm{Sa}, \mathrm{BeLP}\right.$, it was proved that the solutions $v_{\varepsilon}$ of the Cauchy problem for a scalar hyperbolic equation

$$
\frac{\partial^{2} v_{\varepsilon}(\mathbf{x}, \tau)}{\partial \tau^{2}}=\operatorname{div} g^{\varepsilon}(\mathbf{x}) \nabla v_{\varepsilon}(\mathbf{x}, \tau)
$$

tend to the solution $v_{0}$ of the homogenized problem in some weak sense; there were no remainder estimates. In [BeLP], similar results were obtained for the solutions of the Cauchy problem for the Schrödinger-type equation

$$
i \frac{\partial u_{\varepsilon}(\mathbf{x}, \tau)}{\partial \tau}=-\operatorname{div} g^{\varepsilon}(\mathbf{x}) \nabla u_{\varepsilon}(\mathbf{x}, \tau) .
$$

However, note that, usually, homogenization problems are considered not in the entire space $\mathbb{R}^{d}$, but in a bounded domain with some classical boundary conditions. We prefer the setting of the entire space, because boundary conditions lead to worse error estimates due to "boundary layer" effects.

In $\mathrm{BaPa}$, homogenization for the Cauchy problem for a matrix hyperbolic equation of a rather general form (including the system of elasticity theory) in a bounded domain $G$ was studied. Under some smoothness conditions on the problem data, estimates for $\mathbf{v}_{\varepsilon}-\mathbf{v}_{0}$ in the $L_{2}(G \times(0, T))$-norm by $C \sqrt{\varepsilon}$ were obtained. These estimates were not uniform with respect to the right-hand side of the equation; the dependence of a constant in the estimate on $T$ remained unknown. Also in $\mathrm{BaPa}$, further terms of the asymptotic expansion of the solution $\mathbf{v}_{\varepsilon}$ in the small parameter were constructed, but we shall not dwell on this.

0.8. The layout of the paper. The paper consists of five chapters. Chapter $1(\S \S 1-3)$ contains the necessary abstract operator-theoretic material. Preliminary material about periodic differential operators is collected in Chapter $2(\S \S 4-6)$, including the description of the class of DO's under consideration, the direct integral expansion, and a description of the effective characteristics. Chapter $3(\S \S 7-10)$ is devoted to threshold approximations for the smoothed operator-valued functions $\exp \left(-i \tau \varepsilon^{-2} \mathcal{A}\right)$ and $\cos \left(\tau \varepsilon^{-1} \mathcal{A}^{1 / 2}\right)$ of the operators $(0.1)$ and $(0.2)$. Estimates $(0.20),(0.21)$, and their analogs for the operators (0.1) are obtained in that chapter (see Theorems 9.1, 9.2, 10.1, and 10.2). In Chapter 4 (§§11-15), homogenization problems for the Schrödinger-type equation and the hyperbolic equation are considered. Here the main results of the paper given in operator terms, i.e., estimates (0.18), (0.19), and their analogs for the operators (0.3), are obtained (see Theorems 12.1, 12.3, 13.1, and 13.3). Next, these results are applied to homogenization of the Cauchy problems (0.10), (0.11), and also to more general problems. The results for the Schrödinger-type equation are collected in $\S 14$, and the results for the hyperbolic equation are collected in $\S 15$. Chapter $5(\S \S 16-18)$ is devoted to applications of the general results to problems of mathematical physics. In $\S \S 16$ and 17, examples of the Schrödinger-type equation are considered (the model example with the operator $\widehat{\mathcal{A}}_{\varepsilon}=-\operatorname{div} g^{\varepsilon}(\mathbf{x}) \nabla$, the Schrödinger equation with the Hamiltonian $\mathcal{H}_{\varepsilon}=-\operatorname{div} \widetilde{g}^{\varepsilon}(\mathbf{x}) \nabla+\varepsilon^{-2} V^{\varepsilon}(\mathbf{x})$, and the two-dimensional Pauli equation). In $\S 18$ we treat hyperbolic problems for the acoustics equation and the system of elasticity theory. Among the examples considered, there are problems with operators of the form (0.4), 
as well as problems with operators of the form (0.3). In the Appendix (§19), we give a simple proof of one of the principal results of the paper in a relaxed form.

0.9. Notation. Let $\mathfrak{H}, \mathfrak{G}$ be separable Hilbert spaces. The symbols $(\cdot, \cdot)_{\mathfrak{H}}$ and $\|\cdot\|_{\mathfrak{H}}$

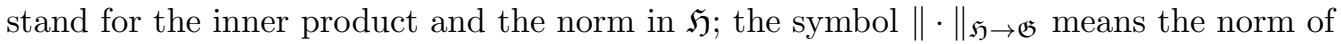
a bounded operator acting from $\mathfrak{H}$ to $\mathfrak{G}$. Sometimes we omit indices if this does not lead to confusion. By $I=I_{\mathfrak{H}}$ we denote the identity operator in $\mathfrak{H}$. If $A: \mathfrak{H} \rightarrow \mathfrak{G}$ is a linear operator, then Dom $A$ denotes its domain. If $\mathfrak{N}$ is a subspace in $\mathfrak{H}$, then $\mathfrak{N}^{\perp}:=\mathfrak{H} \ominus \mathfrak{N}$. If $P$ is the orthogonal projection of $\mathfrak{H}$ onto $\mathfrak{N}$, then $P^{\perp}$ is the orthogonal projection onto $\mathfrak{N}^{\perp}$. The symbol $\langle\cdot, \cdot\rangle=\langle\cdot, \cdot\rangle_{\mathbb{C}^{n}}$ stands for the standard inner product in $\mathbb{C}^{n}$, and $|\cdot|$ stands for the norm of a vector in $\mathbb{C}^{n} ; \mathbf{1}_{n}$ is the unit $(n \times n)$-matrix.

Next, $\mathbf{x}=\left(x^{1}, \ldots, x^{d}\right) \in \mathbb{R}^{d}, i D_{j}=\partial_{j}=\partial / \partial x^{j}, j=1, \ldots, d, \nabla=\operatorname{grad}=$ $\left(\partial_{1}, \ldots, \partial_{d}\right)$, and $\mathbf{D}=-i \nabla=\left(D_{1}, \ldots, D_{d}\right)$.

The classes $L_{p}$ of $\mathbb{C}^{n}$-valued functions in a domain $\mathcal{O} \subseteq \mathbb{R}^{d}$ are denoted by $L_{p}\left(\mathcal{O} ; \mathbb{C}^{n}\right)$, $1 \leq p \leq \infty$. The vector Sobolev classes of order $s$ with integrability index $p$ in a domain $\mathcal{O} \subseteq \mathbb{R}^{d}$ are denoted by $W_{p}^{s}\left(\mathcal{O} ; \mathbb{C}^{n}\right)$. For $p=2$ we use the notation $H^{s}\left(\mathcal{O} ; \mathbb{C}^{n}\right), s \in \mathbb{R}$. If $n=1$, we write simply $L_{p}(\mathcal{O}), W_{p}^{s}(\mathcal{O}), H^{s}(\mathcal{O})$, etc., but sometimes we use such simpler notation for the spaces of vector-valued or matrix-valued functions.

By $C, c, \mathcal{C}, \mathfrak{C}$ (probably, with indices and marks) we denote various constants in the estimates.

\section{Chapter 1. Operator families admitting factorization. Abstract OPERATOR-THEORETIC METHOD}

In this chapter $(\S \S 1-3)$, we present the facts of the abstract theory of selfadjoint operators that are basic for further constructions. These facts supplement the material given in the papers [BSu2, Chapter 1]; BSu3]; [BSu5, Chapter 1]; [Su2, §1, 2]. As in the previous papers, we study factorized operator families $A(t)$ quadratic with respect to the parameter $t \in \mathbb{R}$. We apply methods of the analytic perturbation theory of the discrete spectrum. Part of the material is borrowed from [BSu2, Chapter 1] and [Su2, §1, 2] and is given without proofs. New facts are presented completely.

$\S 1$ contains necessary preliminaries. The main results of Chapter 1 concern approximations of the operator-valued functions of $A(t)$ (the exponential $\exp (-i \tau A(t)$ ) and the cosine $\cos \left(\tau A(t)^{1 / 2}\right)$ ) and are contained in $\S \S 2$ and 3 . The results for the operator exponential are collected in Subsections 2.1, 2.4, 3.2, and 3.3; the results for the operator cosine are collected in Subsections 2.3, 2.5, 3.4, and 3.5.

\section{$\S 1$. Preliminaries}

The content of this section is borrowed from BSu2, Chapter 1].

1.1. Quadratic operator pencils. Let $\mathfrak{H}$ and $\mathfrak{H}_{*}$ be complex separable Hilbert spaces. Suppose that the operator $X_{0}: \mathfrak{H} \rightarrow \mathfrak{H}_{*}$ is densely defined and closed, and that the operator $X_{1}: \mathfrak{H} \rightarrow \mathfrak{H}_{*}$ is bounded. Consider the operator $X(t)=X_{0}+t X_{1}, t \in \mathbb{R}$, on the domain Dom $X(t):=\operatorname{Dom} X_{0}$. The family of selfadjoint (and nonnegative) operators

$$
A(t):=X(t)^{*} X(t), \quad t \in \mathbb{R},
$$

in $\mathfrak{H}$ is our main object. The operator (1.1) is generated by the closed quadratic form $\|X(t) u\|_{\mathfrak{H}_{*}}^{2}, u \in \operatorname{Dom} X_{0}$. We denote $A_{0}:=A(0)=X_{0}^{*} X_{0}$,

$$
\mathfrak{N}:=\operatorname{Ker} A_{0}=\operatorname{Ker} X_{0}, \quad \mathfrak{N}_{*}:=\operatorname{Ker} X_{0}^{*} .
$$

Assume that the point $\lambda_{0}=0$ is an isolated point in the spectrum of $A_{0}$ and that

$$
0<n:=\operatorname{dim} \mathfrak{N}<\infty, \quad n \leq n_{*}:=\operatorname{dim} \mathfrak{N}_{*} \leq \infty
$$


Let $F(t, s)$ denote the spectral projection of the operator $A(t)$ for the closed interval $[0, s]$. We put $\mathfrak{F}(t, s):=F(t, s) \mathfrak{H}$ and fix a number $\delta>0$ such that $8 \delta<d^{0}$, where $d^{0}$ is the distance from the point $\lambda_{0}=0$ to the rest of the spectrum of $A_{0}$. We shall often write $F(t)$ instead of $F(t, \delta)$ and $\mathfrak{F}(t)$ instead of $\mathfrak{F}(t, \delta)$. As was shown in BSu2, Chapter $1,(1.3)]$, we have

$$
F(t, \delta)=F(t, 3 \delta), \quad \operatorname{rank} F(t, \delta)=n, \quad|t| \leq t^{0}:=\delta^{1 / 2}\left\|X_{1}\right\|^{-1} .
$$

Let $P$ and $P_{*}$ denote the orthogonal projections of $\mathfrak{H}$ onto $\mathfrak{N}$ and of $\mathfrak{H}_{*}$ onto $\mathfrak{N}_{*}$, respectively.

1.2. The operator $R$. Let $\mathcal{D}:=\operatorname{Dom} X_{0} \cap \mathfrak{N}^{\perp}$, and let $z \in \mathfrak{H}_{*}$. Consider the equation $X_{0}^{*}\left(X_{0} \psi-z\right)=0$ for an element $\psi \in \mathcal{D}$. This equation is understood in the weak sense:

$$
\left(X_{0} \psi, X_{0} \zeta\right)_{\mathfrak{H}_{*}}=\left(z, X_{0} \zeta\right)_{\mathfrak{H}_{*}}, \quad \zeta \in \mathcal{D}
$$

cf. BSu2, Chapter 1, (1.7)]. The solution $\psi$ exists and is unique. Now, let $\omega \in \mathfrak{N}$ and let $z=-X_{1} \omega$. The element $\psi \in \mathcal{D}$ satisfying (1.3) (with this $z$ ) is denoted by $\psi(\omega)$. We introduce a linear operator $R: \mathfrak{N} \rightarrow \mathfrak{N}_{*}$ (see [BSu2, Chapter 1, Subsection 1.2]):

$$
R \omega=X_{0} \psi(\omega)+X_{1} \omega \in \mathfrak{N}_{*}, \quad \omega \in \mathfrak{N} .
$$

Another description of $R$ is given by the formula $R=\left.P_{*} X_{1}\right|_{\mathfrak{N}}$.

1.3. The spectral germ $S$ of the family $A(t)$ at $t=0$. The selfadjoint operator

$$
S:=R^{*} R: \mathfrak{N} \rightarrow \mathfrak{N}
$$

is called the spectral germ of the operator family (1.1) at $t=0$ (see BSu2, Chapter 1, Subsection 1.3]). The germ satisfies the relation $S=\left.P X_{1}^{*} P_{*} X_{1}\right|_{\mathfrak{N}}$ and the estimate

$$
\|S\| \leq\left\|X_{1}\right\|^{2} \text {. }
$$

The spectral germ $S$ is said to be nondegenerate if Ker $S=\{0\}$.

By the general analytic perturbation theory (see [Ka]), for $|t| \leq t^{0}$ there exist realanalytic functions $\lambda_{l}(t)$ (the branches of eigenvalues) and real-analytic $\mathfrak{H}$-valued functions $\varphi_{l}(t)$ (the branches of eigenvectors) such that

$$
A(t) \varphi_{l}(t)=\lambda_{l}(t) \varphi_{l}(t), \quad l=1, \ldots, n, \quad|t| \leq t^{0},
$$

and the $\varphi_{l}(t), l=1, \ldots, n$, form an orthonormal basis in $\mathfrak{F}(t)$. If $t_{*}$ is sufficiently small, then for $|t| \leq t_{*}$ we have the following convergent power series expansions:

$$
\begin{array}{ll}
\lambda_{l}(t)=\gamma_{l} t^{2}+\mu_{l} t^{3}+\cdots, & \gamma_{l} \geq 0, \mu_{l} \in \mathbb{R}, l=1, \ldots, n, \\
\varphi_{l}(t)=\omega_{l}+t \varphi_{l}^{(1)}+t^{2} \varphi_{l}^{(2)}+\cdots, & l=1, \ldots, n .
\end{array}
$$

The elements $\omega_{l}:=\varphi_{l}(0), l=1, \ldots, n$, form an orthonormal basis in $\mathfrak{N}$.

As was shown in [BSu2, Chapter 1, Proposition 1.6], the numbers $\gamma_{l}$ and the elements $\omega_{l}$ defined by (1.5)-(1.7) are eigenvalues and eigenvectors (respectively) of $S$ :

$$
S \omega_{l}=\gamma_{l} \omega_{l}, \quad l=1, \ldots, n .
$$

Relations (1.8) give an independent (spectral) definition of the germ. The nondegeneracy of the germ is equivalent to the inequalities

$$
\gamma_{l} \geq c_{*}>0, \quad l=1, \ldots, n,
$$

with some $c_{*}>0$. 
1.4. Threshold approximations. As was shown in BSu2, Chapter 1, Theorems 4.1 and 4.3], we have the following approximations for the spectral projection $F(t)=F(t, \delta)$ and for the operator $A(t) F(t)$ :

$$
\begin{aligned}
& F(t)-P=\Phi(t), \quad\|\Phi(t)\| \leq C_{1}|t|, \quad|t| \leq t^{0}, \\
& A(t) F(t)-t^{2} S P=\Psi(t), \quad\|\Psi(t)\| \leq C_{2}|t|^{3}, \quad|t| \leq t^{0} .
\end{aligned}
$$

Here $P$ is the orthogonal projection of $\mathfrak{H}$ onto $\mathfrak{N}$, and $S$ is the spectral germ of the operator family $A(t)$ at $t=0$. The number $t^{0}$ is defined by (1.2), and the constants $C_{1}$ and $C_{2}$ are given by

$$
C_{1}=\beta_{1} \delta^{-1 / 2}\left\|X_{1}\right\|, \quad C_{2}=\beta_{2} \delta^{-1 / 2}\left\|X_{1}\right\|^{3},
$$

where $\beta_{1}$ and $\beta_{2}$ are absolute constants admitting an explicit control.

Note that (1.11) and (1.4) imply that

$$
\|A(t) F(t)\| \leq C_{3} t^{2}, \quad|t| \leq t^{0},
$$

where

$$
C_{3}:=\left\|X_{1}\right\|^{2}+C_{2} t^{0}=\left(1+\beta_{2}\right)\left\|X_{1}\right\|^{2} .
$$

The last relation in (1.14) follows from (1.2) and (1.12).

§2. Threshold approximations For the operators $e^{-i \tau A(t)}$ AND $\cos \left(\tau A(t)^{1 / 2}\right)$

2.1. Approximation for $e^{-i \tau A(t)}$. The following statement was proved in $\mathrm{BSu} 2$, Chapter 1, Theorem 4.6] on the basis of the threshold approximations (1.10), (1.11).

Theorem 2.1. We have

$$
\left\|e^{-i \tau A(t)} F(t)-e^{-i \tau t^{2} S P} P\right\| \leq C_{1}|t|+C_{2}|\tau||t|^{3}, \quad \tau \in \mathbb{R}, \quad|t| \leq t^{0},
$$

where $t^{0}$ is defined by (1.2), and $C_{1}, C_{2}$ are defined by (1.12).

For the reader's convenience, we repeat here the deduction of estimate (2.1) from (1.10) and (1.11).

Proof. We put

$$
\begin{aligned}
& E(\tau):=e^{-i \tau A(t)} F(t)-e^{-i \tau t^{2} S P} P, \\
& \Sigma(\tau):=e^{i \tau t^{2} S P} E(\tau)=e^{i \tau t^{2} S P} F(t) e^{-i \tau A(t)}-P .
\end{aligned}
$$

Note that $\Sigma(0)=F(t)-P=\Phi(t)$. Next,

$$
\frac{d \Sigma(\tau)}{d \tau}=i e^{i \tau t^{2} S P}\left(t^{2} S P-A(t) F(t)\right) F(t) e^{-i \tau A(t)},
$$

whence $\left\|\Sigma^{\prime}(\tau)\right\|=\|\Psi(t) F(t)\| \leq\|\Psi(t)\|$. Combining this with (2.2), (1.10), and (1.11), we obtain

$$
\|E(\tau)\|=\|\Sigma(\tau)\| \leq\|\Phi(t)\|+|\tau|\|\Psi(t)\| \leq C_{1}|t|+C_{2}|\tau||t|^{3}, \quad|t| \leq t^{0} .
$$


2.2. Approximation for $A(t)^{1 / 2} F(t)$. Below we assume the following additional condition on the operator family $A(t)$ (cf. [BSu2, Chapter 1, (5.1)]).

Condition 2.2. For some $c_{*}>0$, we have

$$
A(t) \geq c_{*} t^{2} I, \quad|t| \leq t^{0} .
$$

Condition 2.2 is equivalent to the following inequalities for the eigenvalues $\lambda_{l}(t), l=$ $1, \ldots, n$, of the operator $A(t)$ (see (1.5)):

$$
\lambda_{l}(t) \geq c_{*} t^{2}, \quad l=1, \ldots, n, \quad c_{*}>0, \quad|t| \leq t^{0} .
$$

Then, by (1.6), inequalities (1.9) are valid with the same constant $c_{*}$ as in (2.3). Therefore (see (1.8)), the germ $S$ is nondegenerate, and

$$
S \geq c_{*} I_{\mathfrak{N}}
$$

Consider the difference

$$
\Xi(t):=A(t)^{1 / 2} F(t)-\left(t^{2} S\right)^{1 / 2} P .
$$

Using the notation $\Phi(t)=F(t)-P$, we transform the expression (2.5) to

(2.6) $\Xi(t)=A(t)^{1 / 2} F(t)(P+\Phi(t))-(F(t)-\Phi(t))\left(t^{2} S\right)^{1 / 2} P=\Xi_{1}(t)+\Xi_{2}(t)+\Xi_{3}(t)$.

The term $\Xi_{1}(t):=A(t)^{1 / 2} F(t) \Phi(t)$ is estimated with the help of (1.10) and (1.13):

$$
\left\|\Xi_{1}(t)\right\| \leq C_{1} C_{3}^{1 / 2} t^{2}, \quad|t| \leq t^{0} .
$$

Using (1.10) and (1.4), we estimate the term $\Xi_{2}(t):=\Phi(t)\left(t^{2} S\right)^{1 / 2} P$ :

$$
\left\|\Xi_{2}(t)\right\| \leq C_{1}\left\|X_{1}\right\| t^{2}, \quad|t| \leq t^{0} .
$$

It remains to estimate the term

$$
\Xi_{3}(t):=A(t)^{1 / 2} F(t) P-F(t)\left(t^{2} S\right)^{1 / 2} P=\left(\left.A(t)^{1 / 2} F(t)\right|_{\mathfrak{N}}-F(t)\left(t^{2} S\right)^{1 / 2}\right) P .
$$

We use a theorem pertaining to estimates for the difference of fractional powers of positive operators (see Theorem 3.3 in [BS]). Now we formulate the required version (a particular case) of this theorem; it suffices to consider the case of bounded operators.

Theorem 2.3. Let $\mathcal{H}_{0}, \mathcal{H}_{1}$ be separable Hilbert spaces, and let $B_{0}: \mathcal{H}_{0} \rightarrow \mathcal{H}_{0}$ and $B_{1}: \mathcal{H}_{1} \rightarrow \mathcal{H}_{1}$ be bounded positive operators. Assume that $J: \mathcal{H}_{0} \rightarrow \mathcal{H}_{1}$ is a bounded operator. Then

$$
2\left\|B_{1}^{1 / 2} J-J B_{0}^{1 / 2}\right\|_{\mathcal{H}_{0} \rightarrow \mathcal{H}_{1}} \leq\left\|B_{1}^{-1 / 4}\left(B_{1} J-J B_{0}\right) B_{0}^{-1 / 4}\right\|_{\mathcal{H}_{0} \rightarrow \mathcal{H}_{1}} .
$$

We apply Theorem 2.3 with $\mathcal{H}_{0}=\mathfrak{N}, \mathcal{H}_{1}=\mathfrak{F}(t), B_{0}=t^{2} S, B_{1}=\left.A(t)\right|_{\mathfrak{F}(t)}$, and $J=\left.F(t)\right|_{\mathfrak{N}}$. If $t \neq 0$ and $|t| \leq t^{0}$, then all conditions of Theorem 2.3 are satisfied (due to Condition 2.2 and estimate (2.4)). Hence, by (2.10), the operator (2.9) satisfies the estimate

$$
\begin{aligned}
2\left\|\Xi_{3}(t)\right\|_{\mathfrak{H} \rightarrow \mathfrak{H}} & =2\left\|\left.A(t)^{1 / 2} F(t)\right|_{\mathfrak{N}}-F(t)\left(t^{2} S\right)^{1 / 2}\right\|_{\mathfrak{N} \rightarrow \mathfrak{F}(t)} \\
& \leq\left\|A(t)^{-1 / 4}\left(\left.A(t) F(t)\right|_{\mathfrak{N}}-F(t) t^{2} S\right)\left(t^{2} S\right)^{-1 / 4}\right\|_{\mathfrak{N} \rightarrow \mathfrak{F}(t)} \\
& \leq\left\|A(t)^{-1 / 4}\right\|_{\mathfrak{H} \rightarrow \mathfrak{H}}\left\|A(t) F(t)-t^{2} S P\right\|_{\mathfrak{H} \rightarrow \mathfrak{H}}\left\|\left(t^{2} S\right)^{-1 / 4}\right\|_{\mathfrak{N} \rightarrow \mathfrak{N}}
\end{aligned}
$$

Combining this with (2.3), (2.4), and (1.11), we obtain

$$
\left\|\Xi_{3}(t)\right\| \leq \frac{1}{2} C_{2} c_{*}^{-1 / 2} t^{2}, \quad|t| \leq t^{0} .
$$

We have used the fact that (2.11) is obviously true for $t=0$, because $\Xi_{3}(0)=0$.

Finally, (2.5)-(2.8) and (2.11) imply the following statement. 
Theorem 2.4. We have

$$
\left\|A(t)^{1 / 2} F(t)-\left(t^{2} S\right)^{1 / 2} P\right\| \leq C_{4} t^{2}, \quad|t| \leq t^{0},
$$

where

$$
C_{4}:=C_{1} C_{3}^{1 / 2}+C_{1}\left\|X_{1}\right\|+\frac{1}{2} C_{2} c_{*}^{-1 / 2} .
$$

2.3. Approximation for the operator $\cos \left(\tau A(t)^{1 / 2}\right) F(t)$.

Theorem 2.5. We have where $t^{0}$ is defined by (1.2), and $C_{1}, C_{4}$ are defined by (1.12), (2.13).

Proof. We argue as in the proof of Theorem 2.1. We put

$$
\begin{aligned}
& \widetilde{E}(\tau):=e^{-i \tau A(t)^{1 / 2}} F(t)-e^{-i \tau\left(t^{2} S\right)^{1 / 2} P} P, \\
& \widetilde{\Sigma}(\tau):=e^{i \tau\left(t^{2} S\right)^{1 / 2} P} \widetilde{E}(\tau)=e^{i \tau\left(t^{2} S\right)^{1 / 2} P} F(t) e^{-i \tau A(t)^{1 / 2}}-P .
\end{aligned}
$$

Then $\widetilde{\Sigma}(0)=F(t)-P=\Phi(t)$ and

$$
\frac{d \widetilde{\Sigma}(\tau)}{d \tau}=i e^{i \tau\left(t^{2} S\right)^{1 / 2} P}\left(\left(t^{2} S\right)^{1 / 2} P-A(t)^{1 / 2} F(t)\right) F(t) e^{-i \tau A(t)^{1 / 2}},
$$

whence $\left\|\widetilde{\Sigma}^{\prime}(\tau)\right\| \leq\left\|A(t)^{1 / 2} F(t)-\left(t^{2} S\right)^{1 / 2} P\right\|$. Using (2.15), (1.10), and (2.12), we get

$$
\|\widetilde{E}(\tau)\|=\|\widetilde{\Sigma}(\tau)\| \leq\|\Phi(t)\|+|\tau|\left\|A(t)^{1 / 2} F(t)-\left(t^{2} S\right)^{1 / 2} P\right\| \leq C_{1}|t|+C_{4}|\tau| t^{2}, \quad|t| \leq t^{0},
$$

which yields (2.14).

2.4. Approximation of the "smoothed" operator $\exp \left(-i \tau \varepsilon^{-2} A(t)\right)$ for small $\varepsilon$. Now we introduce a parameter $\varepsilon>0$. We need to study the behavior of the operator $\exp \left(-i \tau \varepsilon^{-2} A(t)\right)$ for small $\varepsilon$. We replace $\tau$ by $\varepsilon^{-2} \tau$ in (2.1). Then

$$
\begin{array}{r}
\left\|\exp \left(-i \tau \varepsilon^{-2} A(t)\right) F(t)-\exp \left(-i \tau \varepsilon^{-2} t^{2} S P\right) P\right\| \leq C_{1}|t|+C_{2}|\tau| \varepsilon^{-2}|t|^{3}, \\
\tau \in \mathbb{R}, \quad \varepsilon>0,|t| \leq t^{0} .
\end{array}
$$

We multiply the operator under the norm sign in (2.16) by $\varepsilon^{3}\left(t^{2}+\varepsilon^{2}\right)^{-3 / 2} P$. The corresponding product, i.e., the operator

$$
\Upsilon(t, \varepsilon, \tau):=\left(\exp \left(-i \tau \varepsilon^{-2} A(t)\right) F(t)-\exp \left(-i \tau \varepsilon^{-2} t^{2} S P\right) P\right) \varepsilon^{3}\left(t^{2}+\varepsilon^{2}\right)^{-3 / 2} P,
$$

admits an estimate of order $\varepsilon$. In applications to differential operators, this corresponds to smoothing of the initial data. By (2.16), the operator (2.17) satisfies the estimate

$$
\begin{array}{r}
\|\Upsilon(t, \varepsilon, \tau)\| \leq\left(C_{1}|t|+C_{2}|\tau| \varepsilon^{-2}|t|^{3}\right) \varepsilon^{3}\left(t^{2}+\varepsilon^{2}\right)^{-3 / 2} \leq\left(\frac{C_{1}}{2}+C_{2}|\tau|\right) \varepsilon, \\
\tau \in \mathbb{R}, \quad \varepsilon>0, \quad|t| \leq t^{0} .
\end{array}
$$

Now we show that an estimate of order $\varepsilon$ remains true if in (2.17) the projection $F(t)$ is replaced by the identity operator $I$. Since $F(t)^{\perp} P=(I-F(t)) P=(P-F(t)) P$, we can use (1.10) to obtain

$$
\begin{aligned}
\left\|\exp \left(-i \tau \varepsilon^{-2} A(t)\right) F(t)^{\perp} \varepsilon^{3}\left(t^{2}+\varepsilon^{2}\right)^{-3 / 2} P\right\| & \leq\|\Phi(t)\| \varepsilon^{3}\left(t^{2}+\varepsilon^{2}\right)^{-3 / 2} \\
\leq C_{1}|t| \varepsilon^{3}\left(t^{2}+\varepsilon^{2}\right)^{-3 / 2} & \leq \frac{C_{1}}{2} \varepsilon, \quad \tau \in \mathbb{R}, \quad \varepsilon>0, \quad|t| \leq t^{0} .
\end{aligned}
$$

Combining this with (2.18), we arrive at the following statement. 
Theorem 2.6. We have

$$
\begin{array}{r}
\left\|\left(\exp \left(-i \tau \varepsilon^{-2} A(t)\right)-\exp \left(-i \tau \varepsilon^{-2} t^{2} S P\right) P\right) \varepsilon^{3}\left(t^{2}+\varepsilon^{2}\right)^{-3 / 2} P\right\| \leq\left(C_{1}+C_{2}|\tau|\right) \varepsilon \\
\tau \in \mathbb{R}, \quad \varepsilon>0, \quad|t| \leq t^{0} .
\end{array}
$$

Here the number $t^{0}$ is defined by (1.2), and the constants $C_{1}, C_{2}$ are defined by (1.12).

2.5. Approximation of the "smoothed" operator $\cos \left(\tau \varepsilon^{-1} A(t)^{1 / 2}\right)$ for small $\varepsilon$. We are interested in the behavior of the operator $\cos \left(\tau \varepsilon^{-1} A(t)^{1 / 2}\right)$ for small $\varepsilon$. Replacing $\tau$ in $(2.14)$ by $\varepsilon^{-1} \tau$, we obtain

$$
\begin{aligned}
\left\|\cos \left(\tau \varepsilon^{-1} A(t)^{1 / 2}\right) F(t)-\cos \left(\tau \varepsilon^{-1}\left(t^{2} S\right)^{1 / 2} P\right) P\right\| & \leq C_{1}|t|+C_{4}|\tau| \varepsilon^{-1} t^{2}, \\
\tau & \in \mathbb{R}, \quad \varepsilon>0, \quad|t| \leq t^{0} .
\end{aligned}
$$

We multiply the operator under the norm sign in $(2.21)$ by $\varepsilon^{2}\left(t^{2}+\varepsilon^{2}\right)^{-1} P$. By $(2.21)$, the corresponding operator satisfies the estimate

$$
\begin{array}{r}
\left\|\left(\cos \left(\tau \varepsilon^{-1} A(t)^{1 / 2}\right) F(t)-\cos \left(\tau \varepsilon^{-1}\left(t^{2} S\right)^{1 / 2} P\right) P\right) \varepsilon^{2}\left(t^{2}+\varepsilon^{2}\right)^{-1} P\right\| \\
\leq\left(C_{1}|t|+C_{4}|\tau| \varepsilon^{-1} t^{2}\right) \varepsilon^{2}\left(t^{2}+\varepsilon^{2}\right)^{-1} \leq\left(\frac{C_{1}}{2}+C_{4}|\tau|\right) \varepsilon \\
\tau \in \mathbb{R}, \quad \varepsilon>0, \quad|t| \leq t^{0} .
\end{array}
$$

As in (2.19), we have

$$
\begin{array}{r}
\left\|\cos \left(\tau \varepsilon^{-1} A(t)^{1 / 2}\right) F(t)^{\perp} \varepsilon^{2}\left(t^{2}+\varepsilon^{2}\right)^{-1} P\right\| \leq C_{1}|t| \varepsilon^{2}\left(t^{2}+\varepsilon^{2}\right)^{-1} \leq \frac{C_{1}}{2} \varepsilon, \\
\tau \in \mathbb{R}, \quad \varepsilon>0, \quad|t| \leq t^{0} .
\end{array}
$$

Combining this with (2.22), we obtain the following statement.

Theorem 2.7. We have

$$
\begin{array}{r}
\left\|\left(\cos \left(\tau \varepsilon^{-1} A(t)^{1 / 2}\right)-\cos \left(\tau \varepsilon^{-1}\left(t^{2} S\right)^{1 / 2} P\right) P\right) \varepsilon^{2}\left(t^{2}+\varepsilon^{2}\right)^{-1} P\right\| \leq\left(C_{1}+C_{4}|\tau|\right) \varepsilon, \\
\tau \in \mathbb{R}, \quad \varepsilon>0, \quad|t| \leq t^{0} .
\end{array}
$$

Here the number $t^{0}$ is defined by (1.2), and the constants $C_{1}, C_{4}$ are defined by (1.12), (2.13).

§3. Threshold approximations in the Case of the family $A(t)=M^{*} \widehat{A}(t) M$

3.1. The operator family of the form $A(t)=M^{*} \widehat{A}(t) M$. The content of this subsection is borrowed from [BSu2, Chapter 1, Subsection 1.5] and [Su2, Subsection 1.3]. Along with the space $\mathfrak{H}$, we consider yet another separable Hilbert space $\widehat{\mathfrak{H}}$. Let $\widehat{X}(t)=\widehat{X}_{0}+t \widehat{X}_{1}: \widehat{\mathfrak{H}} \rightarrow \mathfrak{H}_{*}$ be an operator family of the same form as $X(t)$. Suppose that $\widehat{X}(t)$ satisfies the conditions of Subsection 1.1. Here, the space $\mathfrak{H}_{*}$ remains the same. Let $M: \mathfrak{H} \rightarrow \widehat{\mathfrak{H}}$ be an isomorphism. Assume that $M$ Dom $X_{0}=\operatorname{Dom} \widehat{X}_{0}$ and $X(t)=\widehat{X}(t) M$; then $X_{0}=\widehat{X}_{0} M$ and $X_{1}=\widehat{X}_{1} M$. In $\widehat{\mathfrak{H}}$, we consider the family of selfadjoint operators $\widehat{A}(t)=\widehat{X}(t) * \widehat{X}(t)$. Then, obviously,

$$
A(t)=M^{*} \widehat{A}(t) M \text {. }
$$

In what follows, all objects corresponding to the family $\widehat{A}(t)$ are marked by "^^". Note that $\widehat{\mathfrak{N}}=M \mathfrak{N}, \widehat{n}=n, \widehat{\mathfrak{N}}_{*}=\mathfrak{N}_{*}, \widehat{n}_{*}=n_{*}$, and $\widehat{P}_{*}=P_{*}$.

Consider the following positive definite operator:

$$
Q:=\left(M M^{*}\right)^{-1}: \widehat{\mathfrak{H}} \rightarrow \widehat{\mathfrak{H}} .
$$


Let $Q_{\widehat{\mathfrak{N}}}$ be the block of the operator $Q$ in the subspace $\widehat{\mathfrak{N}}$, i.e.,

$$
Q_{\widehat{\mathfrak{N}}}=\left.\widehat{P} Q\right|_{\widehat{\mathfrak{N}}}: \widehat{\mathfrak{N}} \rightarrow \widehat{\mathfrak{N}} \text {. }
$$

Obviously, $Q_{\widehat{\mathfrak{N}}}$ is an isomorphism in $\widehat{\mathfrak{N}}$.

As was shown in [Su2, Proposition 1.2], the orthogonal projection $P$ of $\mathfrak{H}$ onto $\mathfrak{N}$ and the orthogonal projection $\widehat{P}$ of $\widehat{\mathfrak{H}}$ onto $\widehat{\mathfrak{N}}$ are related by the formula

$$
P=M^{-1}\left(Q_{\widehat{\mathfrak{N}}}\right)^{-1} \widehat{P}\left(M^{*}\right)^{-1} .
$$

Let $\widehat{S}: \widehat{\mathfrak{N}} \rightarrow \widehat{\mathfrak{N}}$ be the spectral germ of the family $\widehat{A}(t)$ at $t=0$, and let $S$ be the germ of the family $A(t)$. The identity

$$
S=\left.P M^{*} \widehat{S} M\right|_{\mathfrak{N}}
$$

was obtained in [BSu2, Chapter 1, Subsection 1.5].

3.2. Approximation of the "bordered" exponential $e^{-i \tau A(t)}$. In this subsection, our goal is to find an approximation for the operator exponential $e^{-i \tau A(t)}$ for the family (3.1) in terms of the germ $\widehat{S}$ of $\widehat{A}(t)$ and the isomorphism $M$. As will be clear below, it is convenient to approximate the exponential "bordered" by appropriate factors.

First, we consider the operator

$$
\Lambda(\tau):=M e^{-i \tau t^{2} S P} P M^{*}: \widehat{\mathfrak{H}} \rightarrow \widehat{\mathfrak{H}}
$$

Using the notation (3.2) and (3.3), we put

$$
M_{0}:=\left(Q_{\widehat{\mathfrak{N}}}\right)^{-1 / 2}: \widehat{\mathfrak{N}} \rightarrow \widehat{\mathfrak{N}}
$$

The following statement is an analog of Proposition 2.3 in [Su2].

Proposition 3.1. For the operator (3.6) acting in $\widehat{\mathfrak{H}}$, we have

$$
\Lambda(\tau)=M_{0} e^{-i \tau t^{2} M_{0} \widehat{S} M_{0}} M_{0} \widehat{P} .
$$

Proof. Suppose $\widehat{\eta} \in \widehat{\mathfrak{H}}$ and $\widehat{\xi}(\tau)=\Lambda(\tau) \widehat{\eta}$. Then $M^{-1} \widehat{\xi}(\tau) \in \mathfrak{N}, \widehat{\xi}(\tau) \in \widehat{\mathfrak{N}}$, and $M^{-1} \widehat{\xi}(\tau)$ is the solution of the Cauchy problem

$$
i \frac{d}{d \tau} M^{-1} \widehat{\xi}(\tau)=t^{2} S M^{-1} \widehat{\xi}(\tau), \quad M^{-1} \widehat{\xi}(0)=P M^{*} \widehat{\eta} .
$$

By (3.5), $S M^{-1} \widehat{\xi}(\tau)=P M^{*} \widehat{S} \widehat{\xi}(\tau)$. Next, from (3.4) and (3.7), it follows that

$$
P M^{*}=M^{-1}\left(Q_{\widehat{\mathfrak{N}}}\right)^{-1} \widehat{P}=M^{-1} M_{0}^{2} \widehat{P} .
$$

Then (3.9) implies that

$$
i \frac{d}{d \tau} \widehat{\xi}(\tau)=t^{2} M_{0}^{2} \widehat{S} \widehat{\xi}(\tau), \quad \widehat{\xi}(0)=M_{0}^{2} \widehat{P} \widehat{\eta},
$$

or equivalently,

$$
i \frac{d}{d \tau} M_{0}^{-1} \widehat{\xi}(\tau)=t^{2} M_{0} \widehat{S} M_{0}\left(M_{0}^{-1} \widehat{\xi}(\tau)\right), \quad M_{0}^{-1} \widehat{\xi}(0)=M_{0} \widehat{P} \widehat{\eta}
$$

Hence, $M_{0}^{-1} \widehat{\xi}(\tau)=e^{-i \tau t^{2} M_{0} \widehat{S} M_{0}} M_{0} \widehat{P} \widehat{\eta}$, which yields (3.8).

We start with estimate (2.1). By (1.10), we obtain

$$
\left\|\left(e^{-i \tau A(t)}-e^{-i \tau t^{2} S P}\right) P\right\|_{\mathfrak{H} \rightarrow \mathfrak{H}} \leq 2 C_{1}|t|+C_{2}|\tau||t|^{3}, \quad \tau \in \mathbb{R}, \quad|t| \leq t^{0} .
$$

This directly implies that

$$
\begin{array}{r}
\left\|M e^{-i \tau A(t)} P M^{*}-M e^{-i \tau t^{2} S P} P M^{*}\right\|_{\widehat{\mathfrak{H}} \rightarrow \widehat{\mathfrak{H}}} \leq\|M\|^{2}\left(2 C_{1}|t|+C_{2}|\tau||t|^{3}\right), \\
\tau \in \mathbb{R}, \quad|t| \leq t^{0} .
\end{array}
$$


By Proposition 3.1 and (3.10), inequality (3.11) can be rewritten as

$$
\begin{array}{r}
\left\|M e^{-i \tau A(t)} M^{-1} M_{0}^{2} \widehat{P}-M_{0} e^{-i \tau t^{2} M_{0} \widehat{S} M_{0}} M_{0} \widehat{P}\right\|_{\widehat{\mathfrak{H}} \rightarrow \widehat{\mathfrak{H}}} \leq\|M\|^{2}\left(2 C_{1}|t|+C_{2}|\tau||t|^{3}\right), \\
\tau \in \mathbb{R}, \quad|t| \leq t^{0} .
\end{array}
$$

Multiplying the operators under the norm sign in (3.12) by $M_{0}^{-2} \widehat{P}$ from the right and using the relation $\left\|M_{0}^{-2}\right\|=\left\|Q_{\widehat{\mathfrak{R}}}\right\| \leq\|Q\|=\left\|M^{-1}\right\|^{2}$, we obtain

$$
\begin{aligned}
& \left\|M e^{-i \tau A(t)} M^{-1} \widehat{P}-M_{0} e^{-i \tau t^{2} M_{0} \widehat{S} M_{0}} M_{0}^{-1} \widehat{P}\right\|_{\widehat{\mathfrak{H}} \rightarrow \widehat{\mathfrak{H}}} \\
& \quad \leq\|M\|^{2}\left\|M^{-1}\right\|^{2}\left(2 C_{1}|t|+C_{2}|\tau||t|^{3}\right), \quad \tau \in \mathbb{R}, \quad|t| \leq t^{0} .
\end{aligned}
$$

3.3. Approximation of the smoothed bordered exponential. Now we replace $\tau$ in (3.13) by $\tau \varepsilon^{-2}$ and multiply the corresponding operator by $\varepsilon^{3}\left(t^{2}+\varepsilon^{2}\right)^{-3 / 2} \widehat{P}$. We have

$$
\begin{aligned}
\|\left(M \exp \left(-i \tau \varepsilon^{-2} A(t)\right) M^{-1} \widehat{P}\right. & \left.-M_{0} \exp \left(-i \tau \varepsilon^{-2} t^{2} M_{0} \widehat{S} M_{0}\right) M_{0}^{-1} \widehat{P}\right) \varepsilon^{3}\left(t^{2}+\varepsilon^{2}\right)^{-3 / 2} \widehat{P} \|_{\widehat{\mathfrak{H}} \rightarrow \widehat{\mathfrak{H}}} \\
& \leq\|M\|^{2}\left\|M^{-1}\right\|^{2}\left(2 C_{1}|t|+C_{2}|\tau| \varepsilon^{-2}|t|^{3}\right) \varepsilon^{3}\left(t^{2}+\varepsilon^{2}\right)^{-3 / 2} \\
& \leq\|M\|^{2}\left\|M^{-1}\right\|^{2}\left(C_{1}+C_{2}|\tau|\right) \varepsilon, \quad \tau \in \mathbb{R}, \quad \varepsilon>0, \quad|t| \leq t^{0} .
\end{aligned}
$$

We have proved the following statement.

Theorem 3.2. Suppose that $A(t)$ and $\widehat{A}(t)$ are related as in (3.1). Let $\widehat{S}$ be the germ of the operator $\widehat{A}(t)$, and let $M_{0}$ be the operator (3.7). Then

$$
\begin{gathered}
\left\|\left(M \exp \left(-i \tau \varepsilon^{-2} A(t)\right) M^{-1}-M_{0} \exp \left(-i \tau \varepsilon^{-2} t^{2} M_{0} \widehat{S} M_{0}\right) M_{0}^{-1} \widehat{P}\right) \varepsilon^{3}\left(t^{2}+\varepsilon^{2}\right)^{-3 / 2} \widehat{P}\right\|_{\widehat{\mathfrak{H}} \rightarrow \widehat{\mathfrak{H}}} \\
\leq\|M\|^{2}\left\|M^{-1}\right\|^{2}\left(C_{1}+C_{2}|\tau|\right) \varepsilon, \quad \tau \in \mathbb{R}, \quad \varepsilon>0, \quad|t| \leq t^{0} .
\end{gathered}
$$

Here the number $t^{0}$ is defined by (1.2), and the constants $C_{1}$ and $C_{2}$ are defined by (1.12).

3.4. Approximation of the "bordered" operator $\cos \left(\tau A(t)^{1 / 2}\right)$. Now, as in Subsection 3.2, we want to approximate the operator $\cos \left(\tau A(t)^{1 / 2}\right)$ for the family (3.1) in terms of the germ $\widehat{S}$ of $\widehat{A}(t)$ and the isomorphism $M$. Here, it is convenient to border this operator by appropriate factors.

First, we consider the operator

$$
\widetilde{\Lambda}(\tau):=M \cos \left(\tau\left(t^{2} S\right)^{1 / 2} P\right) P M^{*}: \widehat{\mathfrak{H}} \rightarrow \widehat{\mathfrak{H}} .
$$

The following statement is similar to Proposition 3.1.

Proposition 3.3. The operator (3.15) acting in $\widehat{\mathfrak{H}}$ admits the following representation:

$$
\widetilde{\Lambda}(\tau)=M_{0} \cos \left(\tau\left(t^{2} M_{0} \widehat{S} M_{0}\right)^{1 / 2}\right) M_{0} \widehat{P} .
$$

Proof. Suppose $\widehat{\eta} \in \widehat{\mathfrak{H}}$ and $\widehat{\xi}(\tau)=\widetilde{\Lambda}(\tau) \widehat{\eta}$. Then $M^{-1} \widehat{\xi}(\tau) \in \mathfrak{N}, \widehat{\xi}(\tau) \in \widehat{\mathfrak{N}}$, and $M^{-1} \widehat{\xi}(\tau)$ is the solution of the Cauchy problem

$$
\frac{d^{2}}{d \tau^{2}} M^{-1} \widehat{\xi}(\tau)=-t^{2} S M^{-1} \widehat{\xi}(\tau),\left.\quad M^{-1} \widehat{\xi}\right|_{\tau=0}=P M^{*} \widehat{\eta},\left.\quad\left(\frac{d}{d \tau} M^{-1} \widehat{\xi}\right)\right|_{\tau=0}=0 .
$$

We use (3.10) and the identity $S M^{-1} \widehat{\xi}(\tau)=P M^{*} \widehat{S} \widehat{\xi}(\tau)$ (see the proof of Proposition 3.1). Then

$$
\frac{d^{2}}{d \tau^{2}} \widehat{\xi}(\tau)=-t^{2} M_{0}^{2} \widehat{S} \widehat{\xi}(\tau), \quad \widehat{\xi}(0)=M_{0}^{2} \widehat{P} \widehat{\eta},\left.\quad\left(\frac{d}{d \tau} \widehat{\xi}\right)\right|_{\tau=0}=0
$$


which is equivalent to the problem

$$
\begin{aligned}
\frac{d^{2}}{d \tau^{2}} M_{0}^{-1} \widehat{\xi}(\tau) & =-t^{2} M_{0} \widehat{S} M_{0}\left(M_{0}^{-1} \widehat{\xi}(\tau)\right), \\
M_{0}^{-1} \widehat{\xi}(0) & =M_{0} \widehat{P} \widehat{\eta},\left.\quad\left(\frac{d}{d \tau} M_{0}^{-1} \widehat{\xi}\right)\right|_{\tau=0}=0 .
\end{aligned}
$$

Hence, $M_{0}^{-1} \widehat{\xi}(\tau)=\cos \left(\tau\left(t^{2} M_{0} \widehat{S} M_{0}\right)^{1 / 2}\right) M_{0} \widehat{P} \widehat{\eta}$, which implies (3.16).

Relations (2.14) and (1.10) show that

$$
\left\|\cos \left(\tau A(t)^{1 / 2}\right) P-\cos \left(\tau\left(t^{2} S\right)^{1 / 2} P\right) P\right\|_{\mathfrak{H} \rightarrow \mathfrak{H}} \leq 2 C_{1}|t|+C_{4}|\tau| t^{2}, \quad \tau \in \mathbb{R}, \quad|t| \leq t^{0} .
$$

This implies the estimate

$$
\begin{aligned}
\| M \cos \left(\tau A(t)^{1 / 2}\right) P M^{*} & -M \cos \left(\tau\left(t^{2} S\right)^{1 / 2} P\right) P M^{*} \|_{\widehat{\mathfrak{H}} \rightarrow \widehat{\mathfrak{H}}} \\
& \leq\|M\|^{2}\left(2 C_{1}|t|+C_{4}|\tau| t^{2}\right), \quad \tau \in \mathbb{R}, \quad|t| \leq t^{0} .
\end{aligned}
$$

By (3.10) and Proposition 3.3, inequality (3.17) can be rewritten in the form

$$
\begin{aligned}
\| M \cos \left(\tau A(t)^{1 / 2}\right) M^{-1} M_{0}^{2} \widehat{P} & -M_{0} \cos \left(\tau\left(t^{2} M_{0} \widehat{S} M_{0}\right)^{1 / 2}\right) M_{0} \widehat{P} \|_{\widehat{\mathfrak{H}} \rightarrow \widehat{\mathfrak{H}}} \\
& \leq\|M\|^{2}\left(2 C_{1}|t|+C_{4}|\tau| t^{2}\right), \quad \tau \in \mathbb{R}, \quad|t| \leq t^{0} .
\end{aligned}
$$

Multiplying the operators under the norm sign in (3.18) by $M_{0}^{-2} \widehat{P}$ from the right, we obtain

$$
\begin{aligned}
\| M \cos \left(\tau A(t)^{1 / 2}\right) M^{-1} \widehat{P} & -M_{0} \cos \left(\tau\left(t^{2} M_{0} \widehat{S} M_{0}\right)^{1 / 2}\right) M_{0}^{-1} \widehat{P} \|_{\widehat{\mathfrak{H}} \rightarrow \widehat{\mathfrak{H}}} \\
& \leq\|M\|^{2}\left\|M^{-1}\right\|^{2}\left(2 C_{1}|t|+C_{4}|\tau| t^{2}\right), \quad \tau \in \mathbb{R}, \quad|t| \leq t^{0} .
\end{aligned}
$$

3.5. Approximation of the smoothed bordered operator $\cos \left(\tau \varepsilon^{-1} A(t)^{1 / 2}\right)$. Now we replace $\tau$ in (3.19) by $\tau \varepsilon^{-1}$ and multiply the corresponding operator by $\varepsilon^{2}\left(t^{2}+\varepsilon^{2}\right)^{-1} \widehat{P}$. Then

$$
\begin{aligned}
\|\left(M \cos \left(\tau \varepsilon^{-1} A(t)^{1 / 2}\right) M^{-1}\right. & \left.\widehat{P}-M_{0} \cos \left(\tau \varepsilon^{-1}\left(t^{2} M_{0} \widehat{S} M_{0}\right)^{1 / 2}\right) M_{0}^{-1} \widehat{P}\right) \varepsilon^{2}\left(t^{2}+\varepsilon^{2}\right)^{-1} \widehat{P} \|_{\widehat{\mathfrak{H}} \rightarrow \widehat{\mathfrak{H}}} \\
& \leq\|M\|^{2}\left\|M^{-1}\right\|^{2}\left(2 C_{1}|t|+C_{4}|\tau| \varepsilon^{-1} t^{2}\right) \varepsilon^{2}\left(t^{2}+\varepsilon^{2}\right)^{-1} \\
& \leq\|M\|^{2}\left\|M^{-1}\right\|^{2}\left(C_{1}+C_{4}|\tau|\right) \varepsilon, \quad \tau \in \mathbb{R}, \quad \varepsilon>0, \quad|t| \leq t^{0} .
\end{aligned}
$$

Thus, we have proved the following theorem.

Theorem 3.4. Assume that $A(t)$ and $\widehat{A}(t)$ satisfy (3.1). Let $\widehat{S}$ be the germ of the operator $\widehat{A}(t)$, and let $M_{0}$ be the operator (3.7). Then

$$
\begin{aligned}
& \|\left(M \cos \left(\tau \varepsilon^{-1} A(t)^{1 / 2}\right) M^{-1}\right.\left.-M_{0} \cos \left(\tau \varepsilon^{-1}\left(t^{2} M_{0} \widehat{S} M_{0}\right)^{1 / 2}\right) M_{0}^{-1} \widehat{P}\right) \varepsilon^{2}\left(t^{2}+\varepsilon^{2}\right)^{-1} \widehat{P} \|_{\widehat{\mathfrak{H}} \rightarrow \widehat{\mathfrak{H}}} \\
& \leq\|M\|^{2}\left\|M^{-1}\right\|^{2}\left(C_{1}+C_{4}|\tau|\right) \varepsilon, \quad \tau \in \mathbb{R}, \quad \varepsilon>0, \quad|t| \leq t^{0} .
\end{aligned}
$$

Here the number $t^{0}$ is defined by (1.2), and the constants $C_{1}$ and $C_{4}$ are defined by (1.12), (2.13). 


\section{Chapter 2. Periodic differential operators in $L_{2}\left(\mathbb{R}^{d} ; \mathbb{C}^{n}\right)$}

In the present chapter $(\S \S 4-6)$, we describe the class of matrix second order DO's admitting a factorization of the form $\mathcal{A}=\mathcal{X}^{*} \mathcal{X}$, where $\mathcal{X}$ is a homogeneous first order DO. This class was singled out and studied in [BSu1, BSu2]. In $\S 4$, we give a detailed description of the class of periodic operators to be considered and introduce the Gelfand transformation. In $\S 5$ we discuss a direct integral expansion for the operator $\mathcal{A}$. In $\S 6$, we define effective characteristics for periodic DO's. A more detailed presentation of this material can be found in BSu2, Chapter 2].

\section{§4. FACTORIZED SECOND ORDER PERIODIC DIFFERENTIAL OPERATORS}

4.1. Factorized second order operators. Let $b(\mathbf{D}): L_{2}\left(\mathbb{R}^{d} ; \mathbb{C}^{n}\right) \rightarrow L_{2}\left(\mathbb{R}^{d} ; \mathbb{C}^{m}\right)$ be a first order homogeneous DO with constant coefficients. We always assume that $m \geq n$. We fix orthonormal bases $\widetilde{\mathbf{e}}_{1}, \ldots, \widetilde{\mathbf{e}}_{n}$ in $\mathbb{C}^{n}, \mathbf{e}_{1}, \ldots, \mathbf{e}_{m}$ in $\mathbb{C}^{m}$, and $\mathbf{e}_{1}^{\prime}, \ldots, \mathbf{e}_{d}^{\prime}$ in $\mathbb{R}^{d}$. Then the operator $b(\mathbf{D})$ corresponds to a symbol $b(\boldsymbol{\xi})$, which is an $(m \times n)$-matrix-valued linear homogeneous function of $\boldsymbol{\xi} \in \mathbb{R}^{d}$. Assume that

$$
\operatorname{rank} b(\boldsymbol{\xi})=n, \quad 0 \neq \boldsymbol{\xi} \in \mathbb{R}^{d} .
$$

Condition (4.1) is equivalent to the condition

$$
\alpha_{0} \mathbf{1}_{n} \leq b(\boldsymbol{\theta})^{*} b(\boldsymbol{\theta}) \leq \alpha_{1} \mathbf{1}_{n}, \quad|\boldsymbol{\theta}|=1, \quad 0<\alpha_{0} \leq \alpha_{1}<\infty,
$$

with some positive constants $\alpha_{0}, \alpha_{1}$.

Suppose that an $(n \times n)$-matrix-valued function $f(\mathbf{x})$ and an $(m \times m)$-matrix-valued function $h(\mathbf{x}), \mathbf{x} \in \mathbb{R}^{d}$, are bounded together with their inverses:

$$
f, f^{-1} \in L_{\infty}\left(\mathbb{R}^{d}\right) ; \quad h, h^{-1} \in L_{\infty}\left(\mathbb{R}^{d}\right)
$$

We consider the DO

$$
\begin{aligned}
\mathcal{X} & :=h b(\mathbf{D}) f: L_{2}\left(\mathbb{R}^{d} ; \mathbb{C}^{n}\right) \rightarrow L_{2}\left(\mathbb{R}^{d} ; \mathbb{C}^{m}\right), \\
\operatorname{Dom} \mathcal{X} & :=\left\{\mathbf{u} \in L_{2}\left(\mathbb{R}^{d} ; \mathbb{C}^{n}\right): f \mathbf{u} \in H^{1}\left(\mathbb{R}^{d} ; \mathbb{C}^{n}\right)\right\} .
\end{aligned}
$$

The operator (4.4) is closed on the domain (4.5). The selfadjoint operator $\mathcal{A}=\mathcal{A}(g, f):=$ $\mathcal{X}^{*} \mathcal{X}$ in $L_{2}\left(\mathbb{R}^{d} ; \mathbb{C}^{n}\right)$ is generated by the closed quadratic form $a[\mathbf{u}, \mathbf{u}]:=\|\mathcal{X} \mathbf{u}\|_{L_{2}\left(\mathbb{R}^{d} ; \mathbb{C}^{m}\right)}^{2}$, $\mathbf{u} \in \operatorname{Dom} \mathcal{X}$. Formally,

$$
\mathcal{A}=\mathcal{A}(g, f)=f(\mathbf{x})^{*} b(\mathbf{D})^{*} g(\mathbf{x}) b(\mathbf{D}) f(\mathbf{x}), \quad g(\mathbf{x}):=h(\mathbf{x})^{*} h(\mathbf{x}) .
$$

By using the Fourier transformation and relations (4.2), (4.3), it is easy to check that

$$
\begin{array}{r}
c_{0} \int_{\mathbb{R}^{d}}|\mathbf{D}(f \mathbf{u})|^{2} d \mathbf{x} \leq a[\mathbf{u}, \mathbf{u}] \leq c_{1} \int_{\mathbb{R}^{d}}|\mathbf{D}(f \mathbf{u})|^{2} d \mathbf{x}, \quad \mathbf{u} \in \operatorname{Dom} \mathcal{X}, \\
c_{0}=\alpha_{0}\left\|h^{-1}\right\|_{L_{\infty}^{-2}}^{-2} \quad c_{1}=\alpha_{1}\|h\|_{L_{\infty}}^{2} .
\end{array}
$$

4.2. The lattices $\Gamma$ and $\widetilde{\Gamma}$. In what follows, we assume that the functions $f, h$ are periodic with respect to some lattice $\Gamma \subset \mathbb{R}^{d}$. Let $\mathbf{a}_{1}, \ldots, \mathbf{a}_{d} \in \mathbb{R}^{d}$ be the basis in $\mathbb{R}^{d}$ that generates the lattice $\Gamma$, i.e.,

$$
\Gamma=\left\{\mathbf{a} \in \mathbb{R}^{d}: \mathbf{a}=\sum_{j=1}^{d} \nu^{j} \mathbf{a}_{j}, \quad \nu^{j} \in \mathbb{Z}\right\} .
$$

Let $\Omega$ be the (elementary) cell of the lattice $\Gamma$ :

$$
\Omega:=\left\{\mathbf{x} \in \mathbb{R}^{d}: \mathbf{x}=\sum_{j=1}^{d} \tau^{j} \mathbf{a}_{j}, \quad 0<\tau^{j}<1\right\} .
$$


The basis $\mathbf{b}^{1}, \ldots, \mathbf{b}^{d}$ in $\mathbb{R}^{d}$ dual to the basis $\mathbf{a}_{1}, \ldots, \mathbf{a}_{d}$ is defined by the relations $\left\langle\mathbf{b}^{i}, \mathbf{a}_{j}\right\rangle=$ $2 \pi \delta_{j}^{i}$. This basis generates the lattice $\widetilde{\Gamma}$ dual to $\Gamma$,

$$
\widetilde{\Gamma}=\left\{\mathbf{b} \in \mathbb{R}^{d}: \mathbf{b}=\sum_{i=1}^{d} \mu_{i} \mathbf{b}^{i}, \quad \mu_{i} \in \mathbb{Z}\right\} .
$$

The cell of $\widetilde{\Gamma}$ can be defined by analogy with (4.8). However, it is more convenient to denote by $\widetilde{\Omega}$ the Brillouin zone

$$
\widetilde{\Omega}=\left\{\mathbf{k} \in \mathbb{R}^{d}:|\mathbf{k}|<|\mathbf{k}-\mathbf{b}|, \quad 0 \neq \mathbf{b} \in \widetilde{\Gamma}\right\} .
$$

Like the cell, the domain $\widetilde{\Omega}$ is a fundamental domain for $\widetilde{\Gamma}$. We use the notation $|\Omega|=$ meas $\Omega,|\widetilde{\Omega}|=$ meas $\widetilde{\Omega}$. Note that $|\Omega||\widetilde{\Omega}|=(2 \pi)^{d}$. Let $r_{0}$ be the radius of the ball inscribed in $\operatorname{clos} \widetilde{\Omega}$. Note that

$$
|\mathbf{b}+\mathbf{k}| \geq r_{0}, \quad 0 \neq \mathbf{b} \in \widetilde{\Gamma}, \quad \mathbf{k} \in \operatorname{clos} \widetilde{\Omega} .
$$

We denote $\mathcal{B}(r)=\left\{\mathbf{k} \in \mathbb{R}^{d}:|\mathbf{k}| \leq r\right\}$.

The discrete Fourier transformation

$$
v(\mathbf{x})=|\Omega|^{-1 / 2} \sum_{\mathbf{b} \in \widetilde{\Gamma}} \hat{v}_{\mathbf{b}} \exp (i\langle\mathbf{b}, \mathbf{x}\rangle), \quad \mathbf{x} \in \Omega,
$$

is associated with the lattice $\Gamma$. This transformation maps $l_{2}(\widetilde{\Gamma})$ onto $L_{2}(\Omega)$ unitarily:

$$
\int_{\Omega}|v(\mathbf{x})|^{2} d \mathbf{x}=\sum_{\mathbf{b} \in \widetilde{\Gamma}}\left|\hat{v}_{\mathbf{b}}\right|^{2} .
$$

Below, $\widetilde{H}^{1}(\Omega)$ denotes the subspace of all functions in $H^{1}(\Omega)$ whose $\Gamma$-periodic extension to $\mathbb{R}^{d}$ belongs to $H_{\text {loc }}^{1}\left(\mathbb{R}^{d}\right)$.

4.3. The Gelfand transformation. Initially, the Gelfand transformation $\mathcal{U}$ is defined on the functions of the Schwartz class $\mathcal{S}$ by the formula

$$
\begin{array}{r}
\widetilde{\mathbf{v}}(\mathbf{k}, \mathbf{x})=(\mathcal{U} \mathbf{v})(\mathbf{k}, \mathbf{x})=|\widetilde{\Omega}|^{-1 / 2} \sum_{\mathbf{a} \in \Gamma} \exp (-i\langle\mathbf{k}, \mathbf{x}+\mathbf{a}\rangle) \mathbf{v}(\mathbf{x}+\mathbf{a}), \\
\mathbf{v} \in \mathcal{S}\left(\mathbb{R}^{d} ; \mathbb{C}^{n}\right), \mathbf{x} \in \Omega, \mathbf{k} \in \widetilde{\Omega} .
\end{array}
$$

Since

$$
\int_{\widetilde{\Omega}} \int_{\Omega}|\widetilde{\mathbf{v}}(\mathbf{k}, \mathbf{x})|^{2} d \mathbf{x} d \mathbf{k}=\int_{\mathbb{R}^{d}}|\mathbf{v}(\mathbf{x})|^{2} d \mathbf{x}, \quad \widetilde{\mathbf{v}}=\mathcal{U} \mathbf{v},
$$

$\mathcal{U}$ extends by continuity to a unitary mapping

$$
\mathcal{U}: L_{2}\left(\mathbb{R}^{d} ; \mathbb{C}^{n}\right) \rightarrow \int_{\widetilde{\Omega}} \oplus L_{2}\left(\Omega ; \mathbb{C}^{n}\right) d \mathbf{k}=: \mathcal{K} .
$$

The relation $\mathbf{v} \in H^{1}\left(\mathbb{R}^{d} ; \mathbb{C}^{n}\right)$ is equivalent to the condition that $\widetilde{\mathbf{v}}(\mathbf{k}, \cdot) \in \widetilde{H}^{1}\left(\Omega ; \mathbb{C}^{n}\right)$ for almost every $\mathbf{k} \in \widetilde{\Omega}$ and

$$
\int_{\widetilde{\Omega}} \int_{\Omega}\left(|(\mathbf{D}+\mathbf{k}) \widetilde{\mathbf{v}}(\mathbf{k}, \mathbf{x})|^{2}+|\widetilde{\mathbf{v}}(\mathbf{k}, \mathbf{x})|^{2}\right) d \mathbf{x} d \mathbf{k}<\infty .
$$

Under the transformation $\mathcal{U}$, the operator of multiplication by a bounded periodic function in $L_{2}\left(\mathbb{R}^{d} ; \mathbb{C}^{n}\right)$ turns into multiplication by the same function on the fibers of the direct integral $\mathcal{K}$ (see (4.11)). On these fibers, the operator $b(\mathbf{D})$ applied to $\mathbf{v} \in H^{1}\left(\mathbb{R}^{d} ; \mathbb{C}^{n}\right)$ turns into the operator $b(\mathbf{D}+\mathbf{k})$ applied to $\widetilde{\mathbf{v}}(\mathbf{k}, \cdot) \in \widetilde{H}^{1}\left(\Omega ; \mathbb{C}^{n}\right)$. 


\section{§5. Direct integral expansion for the operator $\mathcal{A}$}

5.1. The forms $a(\mathbf{k})$ and the operators $\mathcal{A}(\mathbf{k})$. Putting $\mathfrak{H}=L_{2}\left(\Omega ; \mathbb{C}^{n}\right)$ and $\mathfrak{H}_{*}=$ $L_{2}\left(\Omega ; \mathbb{C}^{m}\right)$, we consider the closed operator $\mathcal{X}(\mathbf{k}): \mathfrak{H} \rightarrow \mathfrak{H}_{*}, \mathbf{k} \in \mathbb{R}^{d}$, defined on the domain

$$
\mathfrak{d}:=\operatorname{Dom} \mathcal{X}(\mathbf{k})=\left\{\mathbf{u} \in \mathfrak{H}: f \mathbf{u} \in \widetilde{H}^{1}\left(\Omega ; \mathbb{C}^{n}\right)\right\}
$$

by the formula $\mathcal{X}(\mathbf{k})=h b(\mathbf{D}+\mathbf{k}) f$. The selfadjoint operator

$$
\mathcal{A}(\mathbf{k}):=\mathcal{X}(\mathbf{k})^{*} \mathcal{X}(\mathbf{k}): \mathfrak{H} \rightarrow \mathfrak{H}, \quad \mathbf{k} \in \mathbb{R}^{d},
$$

is generated by the closed quadratic form

$$
a(\mathbf{k})[\mathbf{u}, \mathbf{u}]:=\|\mathcal{X}(\mathbf{k}) \mathbf{u}\|_{\mathfrak{H}_{*}}^{2}, \quad \mathbf{u} \in \mathfrak{d} .
$$

It is easily seen that

$$
c_{0} \int_{\Omega}|(\mathbf{D}+\mathbf{k}) \mathbf{v}|^{2} d \mathbf{x} \leq a(\mathbf{k})[\mathbf{u}, \mathbf{u}] \leq c_{1} \int_{\Omega}|(\mathbf{D}+\mathbf{k}) \mathbf{v}|^{2} d \mathbf{x}, \quad \mathbf{v}=f \mathbf{u} \in \widetilde{H}^{1}\left(\Omega ; \mathbb{C}^{n}\right)
$$

(see [BSu2, Chapter 2, (2.6)]). Here $c_{0}$ and $c_{1}$ are defined by (4.7). From (5.1) and the compactness of the embedding of $\widetilde{H}^{1}\left(\Omega ; \mathbb{C}^{n}\right)$ in $\mathfrak{H}$, it follows that the spectrum of $\mathcal{A}(\mathbf{k})$ is discrete. Observe also that the resolvent of the operator $\mathcal{A}(\mathbf{k})$ is compact and depends on $\mathbf{k} \in \mathbb{R}^{d}$ continuously (in the operator norm).

Let

$$
\mathfrak{N}:=\operatorname{Ker} \mathcal{A}(0)=\operatorname{Ker} \mathcal{X}(0)
$$

Relations (5.1) with $\mathbf{k}=0$ show that

$$
\mathfrak{N}=\left\{\mathbf{u} \in L_{2}\left(\Omega ; \mathbb{C}^{n}\right): f \mathbf{u}=\mathbf{c} \in \mathbb{C}^{n}\right\}, \quad \operatorname{dim} \mathfrak{N}=n .
$$

\subsection{The band functions. Let}

$$
E_{1}(\mathbf{k}) \leq E_{2}(\mathbf{k}) \leq \cdots \leq E_{j}(\mathbf{k}) \leq \cdots, \quad \mathbf{k} \in \mathbb{R}^{d},
$$

be the consecutive eigenvalues of the operator $\mathcal{A}(\mathbf{k})$ (counted with multiplicities). The band functions $E_{j}(\mathbf{k})$ are continuous and $\widetilde{\Gamma}$-periodic.

As was shown in [BSu2, Chapter 2, Subsection 2.2] (by simple variational arguments), the band functions satisfy the following estimates:

$$
\begin{array}{ll}
E_{j}(\mathbf{k}) \geq c_{*}|\mathbf{k}|^{2}, & j=1, \ldots, n, \mathbf{k} \in \operatorname{clos} \widetilde{\Omega}, \\
E_{n+1}(\mathbf{k}) \geq c_{*} r_{0}^{2}, & \mathbf{k} \in \operatorname{clos} \widetilde{\Omega}, \\
E_{n+1}(0) \geq 4 c_{*} r_{0}^{2} . &
\end{array}
$$

Here

$$
c_{*}=\alpha_{0}\left\|f^{-1}\right\|_{L_{\infty}}^{-2}\left\|h^{-1}\right\|_{L_{\infty}}^{-2} .
$$

5.3. The direct integral for the operator $\mathcal{A}$. The operators $\mathcal{A}(\mathbf{k})$ allow us to partially diagonalize the operator $\mathcal{A}$ in the direct integral $\mathcal{K}$ (see (4.11)). Let $\widetilde{\mathbf{u}}=\mathcal{U} \mathbf{u}$ and $\mathbf{u} \in \operatorname{Dom} a$. Then

$$
\begin{aligned}
& \widetilde{\mathbf{u}}(\mathbf{k}, \cdot) \in \mathfrak{d} \quad \text { for a.e. } \mathbf{k} \in \widetilde{\Omega} \\
& a[\mathbf{u}, \mathbf{u}]=\int_{\widetilde{\Omega}} a(\mathbf{k})[\widetilde{\mathbf{u}}(\mathbf{k}, \cdot), \widetilde{\mathbf{u}}(\mathbf{k}, \cdot)] d \mathbf{k} .
\end{aligned}
$$

Conversely, if $\widetilde{\mathbf{u}} \in \mathcal{K}$ satisfies (5.9) and the integral in (5.10) is finite, then $\mathbf{u} \in \operatorname{Dom} a$ and (5.10) is valid. The above arguments show that, in the direct integral $\mathcal{K}$, the operator $\mathcal{A}$ 
turns into multiplication by the operator-valued function $\mathcal{A}(\mathbf{k}), \mathbf{k} \in \widetilde{\Omega}$. All this can be expressed briefly:

$$
\mathcal{U} \mathcal{A} \mathcal{U}^{-1}=\int_{\widetilde{\Omega}} \oplus \mathcal{A}(\mathbf{k}) d \mathbf{k}
$$

Formula (5.11) implies that the spectrum of $\mathcal{A}$ is a union of segments (spectral bands) that are the ranges of the band functions (5.4).

By (5.2) and (5.3), we have

$$
\min _{\mathbf{k} \in \mathbb{R}^{d}} E_{j}(\mathbf{k})=E_{j}(0)=0, \quad j=1, \ldots, n .
$$

Consequently, the bottom of the spectrum of $\mathcal{A}$ coincides with the point $\lambda_{0}=0$, i.e., $\inf \operatorname{spec} \mathcal{A}=0$. The first $n$ spectral bands of the operator $\mathcal{A}$ overlap and have the common bottom $\lambda_{0}=0$, while the $(n+1)$ th band is separated from zero (see (5.6)).

5.4. Incorporation of the operators $\mathcal{A}(\mathbf{k})$ into the pattern of $\S 1$. For $\mathbf{k} \in \mathbb{R}^{d}$ we put $\mathbf{k}=t \boldsymbol{\theta}, t=|\mathbf{k}|,|\boldsymbol{\theta}|=1$, and view $t$ as the main perturbation parameter. At the same time, all our constructions will depend on the additional parameter $\boldsymbol{\theta}$. This dependence will often be reflected in the notation.

We shall apply the method described in $\S 1$ of Chapter 1 . We put $\mathfrak{H}=L_{2}\left(\Omega ; \mathbb{C}^{n}\right)$, $\mathfrak{H}_{*}=L_{2}\left(\Omega ; \mathbb{C}^{m}\right)$. The role of $X(t)$ is played by the operator

$$
X(t, \boldsymbol{\theta})=\mathcal{X}(t \boldsymbol{\theta})=h b(\mathbf{D}+t \boldsymbol{\theta}) f, \quad \operatorname{Dom} X(t, \boldsymbol{\theta})=\mathfrak{d} .
$$

We have $X(t, \boldsymbol{\theta})=X_{0}+t X_{1}(\boldsymbol{\theta})$, where $X_{0}=\mathcal{X}(0)=h b(\mathbf{D}) f$, Dom $X_{0}=\mathfrak{d}$, and $X_{1}(\boldsymbol{\theta})=h b(\boldsymbol{\theta}) f$. Finally, the role of $A(t)$ is played by the operator $A(t, \boldsymbol{\theta})=\mathcal{A}(t \boldsymbol{\theta})$. Next, $A(t, \boldsymbol{\theta})=X(t, \boldsymbol{\theta})^{*} X(t, \boldsymbol{\theta})$. By (5.2) and (5.3), we have $\mathfrak{N}=\operatorname{Ker} X_{0}=\operatorname{Ker} \mathcal{X}(0)$, $\operatorname{dim} \mathfrak{N}=n$. The inequality $m \geq n$ guarantees that $n \leq n_{*}$ (see [BSu2, Chapter 2, §3]).

In accordance with (5.7), the distance $d^{0}$ from the point $\lambda_{0}=0$ to the rest of the spectrum of $\mathcal{A}(0)$ is equal to $E_{n+1}(0)$ and satisfies the estimate

$$
d^{0} \geq 4 c_{*} r_{0}^{2} .
$$

Here $c_{*}$ is the constant (5.8). In Subsection 1.1, it was required that $\delta$ be chosen so that $\delta<d^{0} / 8$. In view of $(5.12)$, now we fix $\delta$ :

$$
\delta=c_{*} r_{0}^{2} / 4=\left(r_{0} / 2\right)^{2} \alpha_{0}\left\|f^{-1}\right\|_{L_{\infty}}^{-2}\left\|h^{-1}\right\|_{L_{\infty}}^{-2} .
$$

Next, by (4.2), the operator $X_{1}(\boldsymbol{\theta})=h b(\boldsymbol{\theta}) f$ satisfies the estimate

$$
\left\|X_{1}(\boldsymbol{\theta})\right\| \leq \alpha_{1}^{1 / 2}\|f\|_{L_{\infty}}\|h\|_{L_{\infty}} .
$$

This allows us to choose $t^{0}$ (see (1.2)) equal not to $\delta^{1 / 2}\left\|X_{1}(\boldsymbol{\theta})\right\|^{-1}$, but to a smaller number independent of $\boldsymbol{\theta}$. Namely, we put

$$
t^{0}=\delta^{1 / 2} \alpha_{1}^{-1 / 2}\|f\|_{L_{\infty}}^{-1}\|h\|_{L_{\infty}}^{-1}=\left(r_{0} / 2\right) \alpha_{0}^{1 / 2} \alpha_{1}^{-1 / 2}\|f\|_{L_{\infty}}^{-1}\left\|f^{-1}\right\|_{L_{\infty}}^{-1}\|h\|_{L_{\infty}}^{-1}\left\|h^{-1}\right\|_{L_{\infty}}^{-1} .
$$

Note that $t^{0} \leq r_{0} / 2$. Thus, $\mathcal{B}\left(t^{0}\right) \subset \mathcal{B}\left(r_{0} / 2\right) \subset \widetilde{\Omega}$. It is essential that $c_{*}$ and $t^{0}$ (see (5.8), (5.15)) do not depend on $\boldsymbol{\theta}$.

5.5. Nondegeneracy of the germ of the family $A(t, \boldsymbol{\theta})$. Below we shall need the following estimate, which is a consequence of the variational estimates (5.5) for eigenvalues of the operator $\mathcal{A}(\mathbf{k})$ :

$$
\mathcal{A}(\mathbf{k})=A(t, \boldsymbol{\theta}) \geq c_{*} t^{2} I, \quad \mathbf{k} \in \operatorname{clos} \widetilde{\Omega}, \quad t=|\mathbf{k}| .
$$


Hence, the operator $A(t, \boldsymbol{\theta})$ satisfies Condition 2.2 with the constant $c_{*}$ defined by (5.8). This implies the nondegeneracy of the germ $S(\boldsymbol{\theta})$ of $A(t, \boldsymbol{\theta})$ (which now depends on the parameter $\boldsymbol{\theta})$ :

$$
S(\boldsymbol{\theta}) \geq c_{*} I_{\mathfrak{N}} .
$$

It is important that the germ is nondegenerate uniformly in $\boldsymbol{\theta}$.

\section{§6. The EFFECTIVE MATRIX AND THE EFFECTIVE OPERATOR}

6.1. The germ of the operator $A(t, \theta)$ in the case where $f=\mathbf{1}_{n}$. In BSu2, Chapter $3, \S 1$, the germ $S(\boldsymbol{\theta})$ of the operator family $A(t, \boldsymbol{\theta})$ was constructed. We need to describe this germ in the case where $f=\mathbf{1}_{n}$. For $f=\mathbf{1}_{n}$, we agree to mark all the corresponding objects by "৯". Then for the operator

$$
\widehat{\mathcal{A}}=\widehat{\mathcal{A}}(g)=b(\mathbf{D})^{*} g(\mathbf{x}) b(\mathbf{D}),
$$

the family $\widehat{\mathcal{A}}(\mathbf{k})$ is denoted by $\widehat{A}(t, \boldsymbol{\theta})$. If $f=\mathbf{1}_{n}$, the kernel (5.3) takes the form

$$
\widehat{\mathfrak{N}}=\left\{\mathbf{u} \in L_{2}\left(\Omega ; \mathbb{C}^{n}\right): \mathbf{u}=\mathbf{c} \in \mathbb{C}^{n}\right\}
$$

i.e., $\widehat{\mathfrak{N}}$ consists of constant vectors. Then the orthogonal projection $\widehat{P}$ of the space $\mathfrak{H}=L_{2}\left(\Omega ; \mathbb{C}^{n}\right)$ onto the subspace $(6.2)$ is the operator of averaging over the cell:

$$
\widehat{P} \mathbf{u}=|\Omega|^{-1} \int_{\Omega} \mathbf{u}(\mathbf{x}) d \mathbf{x} .
$$

As was shown in BSu2, Chapter 3, §1], the spectral germ $\widehat{S}(\boldsymbol{\theta})$ of the family $\widehat{A}(t, \boldsymbol{\theta})$ is the operator acting in the subspace $\widehat{\mathfrak{N}}$ and given by

$$
\widehat{S}(\boldsymbol{\theta})=b(\boldsymbol{\theta})^{*} g^{0} b(\boldsymbol{\theta}), \quad \boldsymbol{\theta} \in \mathbb{S}^{d-1},
$$

where $b(\boldsymbol{\theta})$ is the symbol of the operator $b(\mathbf{D})$, and $g^{0}$ is the so-called effective matrix. The constant positive $(m \times m)$-matrix $g^{0}$ is defined as follows. Let $\mathbf{C} \in \mathbb{C}^{m}$, and let $\mathbf{v}_{\mathbf{C}} \in \widetilde{H}^{1}\left(\Omega ; \mathbb{C}^{n}\right)$ be the (weak) solution of the equation

$$
b(\mathbf{D})^{*} g(\mathbf{x})\left(b(\mathbf{D}) \mathbf{v}_{\mathbf{C}}(\mathbf{x})+\mathbf{C}\right)=0 .
$$

Then

$$
g^{0} \mathbf{C}=|\Omega|^{-1} \int_{\Omega} g(\mathbf{x})\left(b(\mathbf{D}) \mathbf{v}_{\mathbf{C}}(\mathbf{x})+\mathbf{C}\right) d \mathbf{x}, \quad \mathbf{C} \in \mathbb{C}^{m} .
$$

6.2. The effective operator. Since the symbol $b(\mathbf{k})$ is homogeneous, formula (6.4) shows that

$$
\widehat{S}(\mathbf{k}):=t^{2} \widehat{S}(\boldsymbol{\theta})=b(\mathbf{k})^{*} g^{0} b(\mathbf{k}), \quad \mathbf{k} \in \mathbb{R}^{d} .
$$

The expression (6.7) is the symbol of the DO

$$
\widehat{\mathcal{A}}^{0}=b(\mathbf{D})^{*} g^{0} b(\mathbf{D}),
$$

called the effective operator for $\widehat{\mathcal{A}}$.

Let $\widehat{\mathcal{A}}^{0}(\mathbf{k})$ be the operator family in $L_{2}\left(\Omega ; \mathbb{C}^{n}\right)$ corresponding to the effective operator (6.8). Then $\widehat{\mathcal{A}}^{0}(\mathbf{k})$ is given by the expression

$$
\widehat{\mathcal{A}}^{0}(\mathbf{k})=b(\mathbf{D}+\mathbf{k})^{*} g^{0} b(\mathbf{D}+\mathbf{k})
$$

with periodic boundary conditions.

Combining this with (6.3) and (6.7), we obtain the identity

$$
\widehat{S}(\mathbf{k}) \widehat{P}=\widehat{\mathcal{A}}^{0}(\mathbf{k}) \widehat{P},
$$

which will be used below. 
6.3. The properties of the effective matrix. Note that (see BSu2, Chapter 3, Theorem 1.5]) the effective matrix $g^{0}$ satisfies the following estimates (known as the Voight-Reuss bracketing):

where

$$
\underline{g} \leq g^{0} \leq \bar{g}
$$

$$
\bar{g}=|\Omega|^{-1} \int_{\Omega} g(\mathbf{x}) d \mathbf{x}, \quad \underline{g}=\left(|\Omega|^{-1} \int_{\Omega} g(\mathbf{x})^{-1} d \mathbf{x}\right)^{-1} .
$$

If $m=n$ (i.e., when the operator admits factorization with square matrices), we always have $g^{0}=\underline{g}$.

\section{Chapter 3. Threshold approximations of the operator-VAlued FunCtions OF PERIODIC DIFFERENTIAL OPERATORS}

This chapter is devoted to threshold approximations for operator-valued functions (the operator exponential and the operator cosine) of periodic DO's. These approximations are obtained on the basis of abstract theorems from Chapter $1 . \S 7$ contains the required approximations for the functions of operators $\widehat{\mathcal{A}}(\mathbf{k})$ acting in $L_{2}\left(\Omega ; \mathbb{C}^{n}\right)$ and depending on the quasimomentum $\mathbf{k}$. In $\S 8$, similar facts are obtained for the case of more general operators $\mathcal{A}(\mathbf{k})$. In $\S \S 9$ and 10, approximations for the operator-valued functions of the operators $\widehat{\mathcal{A}}$ and $\mathcal{A}$ acting in $L_{2}\left(\mathbb{R}^{d} ; \mathbb{C}^{n}\right)$ are deduced from the results of $\delta \S 7$ and 8 . Results for the operator exponential are collected in Subsections 7.1-7.3, 8.1, 8.2, 9.1, and 10.1, and those for the operator cosine are collected in Subsections 7.4, 7.5, 8.3, 8.4, 9.2 , and 10.2 .

\section{§7. Approximations for the Smoothed operators}

$$
\exp \left(-i \tau \varepsilon^{-2} \widehat{\mathcal{A}}(\mathbf{k})\right) \text { AND } \cos \left(\tau \varepsilon^{-1} \widehat{\mathcal{A}}(\mathbf{k})^{1 / 2}\right)
$$

7.1. Approximation of the smoothed operator $\exp \left(-i \tau \varepsilon^{-2} \widehat{\mathcal{A}}(\mathbf{k})\right)$ for $|\mathbf{k}| \leq \widehat{t}^{0}$. Consider the operator $\mathcal{H}_{0}=-\Delta$ in $L_{2}\left(\mathbb{R}^{d} ; \mathbb{C}^{n}\right)$. The operator $\mathcal{H}_{0}$ is expanded in the direct integral of operators $\mathcal{H}_{0}(\mathbf{k})$ acting in $L_{2}\left(\Omega ; \mathbb{C}^{n}\right)$. The operator $\mathcal{H}_{0}(\mathbf{k})$ is defined by the differential expression $|\mathbf{D}+\mathbf{k}|^{2}$ with periodic boundary conditions. We introduce the notation

$$
\mathcal{R}(\mathbf{k}, \varepsilon):=\varepsilon^{2}\left(\mathcal{H}_{0}(\mathbf{k})+\varepsilon^{2} I\right)^{-1} .
$$

Clearly, we have

$$
\begin{aligned}
\mathcal{R}(\mathbf{k}, \varepsilon) \widehat{P} & =\varepsilon^{2}\left(t^{2}+\varepsilon^{2}\right)^{-1} \widehat{P}, \\
\mathcal{R}(\mathbf{k}, \varepsilon)^{3 / 2} \widehat{P} & =\varepsilon^{3}\left(t^{2}+\varepsilon^{2}\right)^{-3 / 2} \widehat{P}, \quad t=|\mathbf{k}| .
\end{aligned}
$$

We apply Theorem 2.6 to the family of operators $\widehat{A}(t, \boldsymbol{\theta})=\widehat{\mathcal{A}}(\mathbf{k})$. By $(6.7)$ and (6.9), we have

$$
\exp \left(-i \tau \varepsilon^{-2} t^{2} \widehat{S}(\boldsymbol{\theta}) \widehat{P}\right) \widehat{P}=\exp \left(-i \tau \varepsilon^{-2} \widehat{\mathcal{A}}^{0}(\mathbf{k})\right) \widehat{P} .
$$

It remains to indicate the constants. In the case where $f=\mathbf{1}_{n}$, the constants (5.8), (5.13), and (5.15) take the form

$$
\begin{aligned}
\widehat{c}_{*} & =\alpha_{0}\left\|h^{-1}\right\|_{L_{\infty}}^{-2}, \\
\widehat{\delta} & =\left(r_{0} / 2\right)^{2} \alpha_{0}\left\|h^{-1}\right\|_{L_{\infty}}^{-2}, \\
\widehat{t}^{0} & =\left(r_{0} / 2\right) \alpha_{0}^{1 / 2} \alpha_{1}^{-1 / 2}\|h\|_{L_{\infty}}^{-1}\left\|h^{-1}\right\|_{L_{\infty}}^{-1} .
\end{aligned}
$$

Using the estimate

$$
\left\|\widehat{X}_{1}(\boldsymbol{\theta})\right\| \leq \alpha_{1}^{1 / 2}\|h\|_{L_{\infty}}
$$


(see (5.14) with $f=\mathbf{1}_{n}$ ), we can replace the precise values of the constants (1.12), which now depend on $\boldsymbol{\theta}$, with the larger values

$$
\begin{aligned}
& \widehat{C}_{1}=\beta_{1}\left(2 / r_{0}\right) \alpha_{1}^{1 / 2} \alpha_{0}^{-1 / 2}\|h\|_{L_{\infty}}\left\|h^{-1}\right\|_{L_{\infty}}, \\
& \widehat{C}_{2}=\beta_{2}\left(2 / r_{0}\right) \alpha_{1}^{3 / 2} \alpha_{0}^{-1 / 2}\|h\|_{L_{\infty}}^{3}\left\|h^{-1}\right\|_{L_{\infty}} .
\end{aligned}
$$

Combining (2.20) with (7.2) and (7.3), we obtain

$$
\begin{aligned}
& \|\left(\exp \left(-i \tau \varepsilon^{-2} \widehat{\mathcal{A}}(\mathbf{k})\right)-\right.\left.\exp \left(-i \tau \varepsilon^{-2} \widehat{\mathcal{A}}^{0}(\mathbf{k})\right)\right) \mathcal{R}(\mathbf{k}, \varepsilon)^{3 / 2} \widehat{P} \|_{L_{2}(\Omega) \rightarrow L_{2}(\Omega)} \\
& \leq\left(\widehat{C}_{1}+\widehat{C}_{2}|\tau|\right) \varepsilon, \quad \tau \in \mathbb{R}, \quad \varepsilon>0, \quad|\mathbf{k}| \leq \widehat{t}^{0} .
\end{aligned}
$$

7.2. Approximation of the smoothed operator $\exp \left(-i \tau \varepsilon^{-2} \widehat{\mathcal{A}}(\mathbf{k})\right)$ for $|\mathbf{k}|>\widehat{t}^{0}$. Our estimates for the operator under the norm sign in (7.9) for $\mathbf{k} \in \operatorname{clos} \widetilde{\Omega} \backslash \mathcal{B}\left(\widehat{t}^{0}\right)$ are rough: each term is estimated separately.

By (7.2), we have

$$
\begin{array}{r}
\left\|\mathcal{R}(\mathbf{k}, \varepsilon)^{3 / 2} \widehat{P}\right\|=\varepsilon^{3}\left(|\mathbf{k}|^{2}+\varepsilon^{2}\right)^{-3 / 2} \leq \varepsilon^{3}\left(\widehat{t}^{0}\right)^{-3} \leq \varepsilon\left(\widehat{t}^{0}\right)^{-3}, \\
0<\varepsilon \leq 1, \quad \mathbf{k} \in \operatorname{clos} \widetilde{\Omega} \backslash \mathcal{B}\left(\widehat{t}^{0}\right) .
\end{array}
$$

This implies that

$$
\begin{array}{r}
\left\|\left(\exp \left(-i \tau \varepsilon^{-2} \widehat{\mathcal{A}}(\mathbf{k})\right)-\exp \left(-i \tau \varepsilon^{-2} \widehat{\mathcal{A}}^{0}(\mathbf{k})\right)\right) \mathcal{R}(\mathbf{k}, \varepsilon)^{3 / 2} \widehat{P}\right\|_{L_{2}(\Omega) \rightarrow L_{2}(\Omega)} \leq 2 \varepsilon\left(\widehat{t}^{0}\right)^{-3} \\
\tau \in \mathbb{R}, \quad 0<\varepsilon \leq 1, \quad \mathbf{k} \in \operatorname{clos} \widetilde{\Omega} \backslash \mathcal{B}\left(\widehat{t}^{0}\right) .
\end{array}
$$

Now, from (7.9) and (7.11) we obtain

$$
\begin{array}{r}
\left\|\left(\exp \left(-i \tau \varepsilon^{-2} \widehat{\mathcal{A}}(\mathbf{k})\right)-\exp \left(-i \tau \varepsilon^{-2} \widehat{\mathcal{A}}^{0}(\mathbf{k})\right)\right) \mathcal{R}(\mathbf{k}, \varepsilon)^{3 / 2} \widehat{P}\right\|_{L_{2}(\Omega) \rightarrow L_{2}(\Omega)} \\
\leq\left(\widehat{C}_{1}+2\left(\widehat{t}^{0}\right)^{-3}+\widehat{C}_{2}|\tau|\right) \varepsilon, \quad \tau \in \mathbb{R}, \quad 0<\varepsilon \leq 1, \quad \mathbf{k} \in \operatorname{clos} \widetilde{\Omega}
\end{array}
$$

7.3. Elimination of the operator $\widehat{P}$. Now we show that, up to an admissible error, the projection $\widehat{P}$ can be replaced by the identity operator under the norm sign in (7.12). For this, we estimate the norm of the operator $\mathcal{R}(\mathbf{k}, \varepsilon)^{3 / 2}(I-\widehat{P})$. Under the discrete Fourier transformation (see (4.10)), the operator $\mathcal{R}(\mathbf{k}, \varepsilon)$ applied to a periodic function $\mathbf{v}(\mathbf{x})$ turns into multiplication of the Fourier coefficients $\hat{\mathbf{v}}_{\mathbf{b}}, \mathbf{b} \in \widetilde{\Gamma}$, by the symbol $\varepsilon^{2}\left(|\mathbf{b}+\mathbf{k}|^{2}+\varepsilon^{2}\right)^{-1}$. The operator $I-\widehat{P}$ makes the zero Fourier coefficient $\hat{\mathbf{v}}_{0}$ equal to zero. Therefore,

$$
\begin{array}{r}
\left\|\mathcal{R}(\mathbf{k}, \varepsilon)^{3 / 2}(I-\widehat{P})\right\|_{L_{2}(\Omega) \rightarrow L_{2}(\Omega)}=\max _{0 \neq \mathbf{b} \in \widetilde{\Gamma}} \varepsilon^{3}\left(|\mathbf{b}+\mathbf{k}|^{2}+\varepsilon^{2}\right)^{-3 / 2} \leq \varepsilon^{3} r_{0}^{-3}, \\
\varepsilon>0, \quad \mathbf{k} \in \operatorname{clos} \widetilde{\Omega} .
\end{array}
$$

Here we have used estimate (4.9). Hence,

$$
\begin{array}{r}
\left\|\left(\exp \left(-i \tau \varepsilon^{-2} \widehat{\mathcal{A}}(\mathbf{k})\right)-\exp \left(-i \tau \varepsilon^{-2} \widehat{\mathcal{A}}^{0}(\mathbf{k})\right)\right) \mathcal{R}(\mathbf{k}, \varepsilon)^{3 / 2}(I-\widehat{P})\right\|_{L_{2}(\Omega) \rightarrow L_{2}(\Omega)} \\
\leq 2 \varepsilon r_{0}^{-3}, \quad \tau \in \mathbb{R}, \quad 0<\varepsilon \leq 1, \quad \mathbf{k} \in \operatorname{clos} \widetilde{\Omega} .
\end{array}
$$

Now, relations (7.12) and (7.14) imply the following result.

Theorem 7.1. For $\tau \in \mathbb{R}, 0<\varepsilon \leq 1$, and $\mathbf{k} \in \operatorname{clos} \widetilde{\Omega}$, we have

$$
\begin{aligned}
\|\left(\exp \left(-i \tau \varepsilon^{-2} \widehat{\mathcal{A}}(\mathbf{k})\right)-\exp \left(-i \tau \varepsilon^{-2} \widehat{\mathcal{A}}^{0}(\mathbf{k})\right)\right) \mathcal{R}(\mathbf{k}, \varepsilon)^{3 / 2} & \|_{L_{2}(\Omega) \rightarrow L_{2}(\Omega)} \\
& \leq\left(\mathcal{C}_{1}+\mathcal{C}_{2}|\tau|\right) \varepsilon
\end{aligned}
$$


where

$$
\mathcal{C}_{1}=\widehat{C}_{1}+2\left(\widehat{t}^{0}\right)^{-3}+2 r_{0}^{-3}, \quad \mathcal{C}_{2}=\widehat{C}_{2} .
$$

The operator $\mathcal{R}(\mathbf{k}, \varepsilon)$ is defined by (7.1), the number $\widehat{t}^{0}$ is given by (7.5), and the constants $\widehat{C}_{1}, \widehat{C}_{2}$ are defined by (7.7), (7.8).

7.4. Approximation of the smoothed operator $\cos \left(\tau \varepsilon^{-1} \widehat{\mathcal{A}}(\mathbf{k})^{1 / 2}\right)$ for $|\mathbf{k}| \leq \widehat{t}^{0}$. We apply Theorem 2.7 to the operator family $\widehat{A}(t, \boldsymbol{\theta})=\widehat{\mathcal{A}}(\mathbf{k})$. By analogy with (7.3), formulas (6.7) and (6.9) show that

$$
\cos \left(\tau \varepsilon^{-1}\left(t^{2} \widehat{S}(\boldsymbol{\theta})\right)^{1 / 2} \widehat{P}\right) \widehat{P}=\cos \left(\tau \varepsilon^{-1} \widehat{\mathcal{A}}^{0}(\mathbf{k})^{1 / 2}\right) \widehat{P} .
$$

It remains to indicate the constants $C_{3}$ and $C_{4}$. Estimate (7.6) shows that, instead of the precise value of the constant (1.14) (which depends on $\boldsymbol{\theta}$ ), it is convenient to take

$$
\widehat{C}_{3}=\left(1+\beta_{2}\right) \alpha_{1}\|h\|_{L_{\infty}}^{2} .
$$

Instead of the precise value of the constant (2.13), we choose

$$
\widehat{C}_{4}=\widehat{C}_{1} \widehat{C}_{3}^{1 / 2}+\widehat{C}_{1} \alpha_{1}^{1 / 2}\|h\|_{L_{\infty}}+\frac{1}{2} \widehat{C}_{2}\left(\widehat{c}_{*}\right)^{-1 / 2} .
$$

Taking (7.4), (7.7), (7.8), and (7.18) into account, we arrive at

$$
\begin{array}{r}
\widehat{C}_{4}=2 \beta_{1}\left(1+\left(1+\beta_{2}\right)^{1 / 2}\right) r_{0}^{-1} \alpha_{1} \alpha_{0}^{-1 / 2}\|h\|_{L_{\infty}}^{2}\left\|h^{-1}\right\|_{L_{\infty}} \\
+\beta_{2} r_{0}^{-1} \alpha_{1}^{3 / 2} \alpha_{0}^{-1}\|h\|_{L_{\infty}}^{3}\left\|h^{-1}\right\|_{L_{\infty}}^{2} .
\end{array}
$$

Applying estimate (2.23) and using (7.2) and (7.17), we obtain

$$
\begin{aligned}
\left\|\left(\cos \left(\tau \varepsilon^{-1} \widehat{\mathcal{A}}(\mathbf{k})^{1 / 2}\right)-\cos \left(\tau \varepsilon^{-1} \widehat{\mathcal{A}}^{0}(\mathbf{k})^{1 / 2}\right)\right) \mathcal{R}(\mathbf{k}, \varepsilon) \widehat{P}\right\|_{L_{2}(\Omega) \rightarrow L_{2}(\Omega)} \\
\leq\left(\widehat{C}_{1}+\widehat{C}_{4}|\tau|\right) \varepsilon, \quad \tau \in \mathbb{R}, \quad \varepsilon>0, \quad|\mathbf{k}| \leq \widehat{t}^{0} .
\end{aligned}
$$

7.5. Approximation of the smoothed operator $\cos \left(\tau \varepsilon^{-1} \widehat{\mathcal{A}}(\mathbf{k})^{1 / 2}\right)$ for $|\mathbf{k}|>\widehat{t}^{0}$. By analogy with (7.11), we have

$$
\begin{aligned}
& \left\|\left(\cos \left(\tau \varepsilon^{-1} \widehat{\mathcal{A}}(\mathbf{k})^{1 / 2}\right)-\cos \left(\tau \varepsilon^{-1} \widehat{\mathcal{A}}^{0}(\mathbf{k})^{1 / 2}\right)\right) \mathcal{R}(\mathbf{k}, \varepsilon) \widehat{P}\right\|_{L_{2}(\Omega) \rightarrow L_{2}(\Omega)} \\
& \quad \leq 2 \varepsilon^{2}\left(\widehat{t}^{0}\right)^{-2} \leq 2 \varepsilon\left(\widehat{t}^{0}\right)^{-2}, \quad \tau \in \mathbb{R}, \quad 0<\varepsilon \leq 1, \quad \mathbf{k} \in \operatorname{clos} \widetilde{\Omega} \backslash \mathcal{B}\left(\widehat{t}^{0}\right) .
\end{aligned}
$$

Now (7.20) and (7.21) imply that

$$
\begin{array}{r}
\left\|\left(\cos \left(\tau \varepsilon^{-1} \widehat{\mathcal{A}}(\mathbf{k})^{1 / 2}\right)-\cos \left(\tau \varepsilon^{-1} \widehat{\mathcal{A}}^{0}(\mathbf{k})^{1 / 2}\right)\right) \mathcal{R}(\mathbf{k}, \varepsilon) \widehat{P}\right\|_{L_{2}(\Omega) \rightarrow L_{2}(\Omega)} \\
\leq\left(\widehat{C}_{1}+2\left(\widehat{t}^{0}\right)^{-2}+\widehat{C}_{4}|\tau|\right) \varepsilon, \quad \tau \in \mathbb{R}, \quad 0<\varepsilon \leq 1, \quad \mathbf{k} \in \operatorname{clos} \widetilde{\Omega} .
\end{array}
$$

The next estimate is similar to (7.14):

$$
\begin{array}{r}
\left\|\left(\cos \left(\tau \varepsilon^{-1} \widehat{\mathcal{A}}(\mathbf{k})^{1 / 2}\right)-\cos \left(\tau \varepsilon^{-1} \widehat{\mathcal{A}}^{0}(\mathbf{k})^{1 / 2}\right)\right) \mathcal{R}(\mathbf{k}, \varepsilon)(I-\widehat{P})\right\|_{L_{2}(\Omega) \rightarrow L_{2}(\Omega)} \leq 2 \varepsilon r_{0}^{-2}, \\
\tau \in \mathbb{R}, \quad 0<\varepsilon \leq 1, \quad \mathbf{k} \in \operatorname{clos} \widetilde{\Omega} .
\end{array}
$$

Finally, estimates (7.22) and (7.23) lead to the following statement.

Theorem 7.2. For $\tau \in \mathbb{R}, 0<\varepsilon \leq 1$, and $\mathbf{k} \in \operatorname{clos} \widetilde{\Omega}$, we have

$$
\left\|\left(\cos \left(\tau \varepsilon^{-1} \widehat{\mathcal{A}}(\mathbf{k})^{1 / 2}\right)-\cos \left(\tau \varepsilon^{-1} \widehat{\mathcal{A}}^{0}(\mathbf{k})^{1 / 2}\right)\right) \mathcal{R}(\mathbf{k}, \varepsilon)\right\|_{L_{2}(\Omega) \rightarrow L_{2}(\Omega)} \leq\left(\mathcal{C}_{3}+\mathcal{C}_{4}|\tau|\right) \varepsilon,
$$

where

$$
\mathcal{C}_{3}=\widehat{C}_{1}+2\left(\widehat{t}^{0}\right)^{-2}+2 r_{0}^{-2}, \quad \mathcal{C}_{4}=\widehat{C}_{4}
$$


The operator $\mathcal{R}(\mathbf{k}, \varepsilon)$ is given by (7.1), the number $\widehat{t}^{0}$ is defined by (7.5), and the constants $\widehat{C}_{1}$ and $\widehat{C}_{4}$ are defined by (7.7) and (7.19).

\section{§8. Approximation of the SMoothed bordered operators $\exp \left(-i \tau \varepsilon^{-2} \mathcal{A}(\mathbf{k})\right)$ AND $\cos \left(\tau \varepsilon^{-1} \mathcal{A}(\mathbf{k})^{1 / 2}\right)$}

8.1. Approximation of the smoothed bordered operator $\exp \left(-i \tau \varepsilon^{-2} \mathcal{A}(\mathbf{k})\right)$ for $|\mathbf{k}| \leq t^{0}$. We study the exponential of the operator $\mathcal{A}(\mathbf{k})=f^{*} \widehat{\mathcal{A}}(\mathbf{k}) f$ by using the method described in $\S 3$. Now $\mathfrak{H}=\widehat{\mathfrak{H}}=L_{2}\left(\Omega ; \mathbb{C}^{n}\right)$, the role of $A(t)$ is played by the operator $A(t, \boldsymbol{\theta})=\mathcal{A}(\mathbf{k})$, and the role of $\widehat{A}(t)$ is played by $\widehat{A}(t, \boldsymbol{\theta})=\widehat{\mathcal{A}}(\mathbf{k})$. The isomorphism $M$ is the operator of multiplication by the matrix-valued function $f(\mathbf{x})$. The operator $Q$ (see $(3.2))$ is the operator of multiplication by the matrix-valued function

$$
Q(\mathbf{x})=\left(f(\mathbf{x}) f(\mathbf{x})^{*}\right)^{-1} .
$$

The block of the operator $Q$ in the kernel $\widehat{\mathfrak{N}}$ (see (6.2)) is the operator of multiplication by the constant matrix

$$
\bar{Q}=\left(\underline{f f^{*}}\right)^{-1}=|\Omega|^{-1} \int_{\Omega}\left(f(\mathbf{x}) f(\mathbf{x})^{*}\right)^{-1} d \mathbf{x} .
$$

Next, $M_{0}$ (see (3.7)) is the operator of multiplication by the constant matrix

$$
f_{0}=(\bar{Q})^{-1 / 2}=\left(\underline{f f^{*}}\right)^{1 / 2} .
$$

The constant $t^{0}$ is given by (5.15). Using (5.13) and (5.14), we can replace the precise values of the constants (1.12) (which depend on $\boldsymbol{\theta}$ ) with the larger values

$$
\begin{aligned}
& C_{1}=2 \beta_{1} r_{0}^{-1} \alpha_{1}^{1 / 2} \alpha_{0}^{-1 / 2}\|h\|_{L_{\infty}}\left\|h^{-1}\right\|_{L_{\infty}}\|f\|_{L_{\infty}}\left\|f^{-1}\right\|_{L_{\infty}}, \\
& C_{2}=2 \beta_{2} r_{0}^{-1} \alpha_{1}^{3 / 2} \alpha_{0}^{-1 / 2}\|h\|_{L_{\infty}}^{3}\left\|h^{-1}\right\|_{L_{\infty}}\|f\|_{L_{\infty}}^{3}\left\|f^{-1}\right\|_{L_{\infty}} .
\end{aligned}
$$

We put

$$
\mathcal{A}^{0}:=f_{0} \widehat{\mathcal{A}}^{0} f_{0}=f_{0} b(\mathbf{D})^{*} g^{0} b(\mathbf{D}) f_{0} .
$$

Let $\mathcal{A}^{0}(\mathbf{k})$ be the corresponding operator family in $L_{2}\left(\Omega ; \mathbb{C}^{n}\right)$. Then $\mathcal{A}^{0}(\mathbf{k})=f_{0} \widehat{\mathcal{A}}^{0}(\mathbf{k}) f_{0}$.

We apply inequality (3.14) to the operator $A(t, \boldsymbol{\theta})=\mathcal{A}(\mathbf{k})$. By (6.7) and (6.9), we have

$$
f_{0} \exp \left(-i \tau \varepsilon^{-2} t^{2} f_{0} \widehat{S}(\boldsymbol{\theta}) f_{0}\right) f_{0}^{-1} \widehat{P}=f_{0} \exp \left(-i \tau \varepsilon^{-2} \mathcal{A}^{0}(\mathbf{k})\right) f_{0}^{-1} \widehat{P} .
$$

Using (7.2), we obtain

$$
\begin{array}{r}
\left\|\left(f \exp \left(-i \tau \varepsilon^{-2} \mathcal{A}(\mathbf{k})\right) f^{-1}-f_{0} \exp \left(-i \tau \varepsilon^{-2} \mathcal{A}^{0}(\mathbf{k})\right) f_{0}^{-1}\right) \mathcal{R}(\mathbf{k}, \varepsilon)^{3 / 2} \widehat{P}\right\|_{L_{2}(\Omega) \rightarrow L_{2}(\Omega)} \\
\leq\|f\|_{L_{\infty}}^{2}\left\|f^{-1}\right\|_{L_{\infty}}^{2}\left(C_{1}+C_{2}|\tau|\right) \varepsilon, \quad \tau \in \mathbb{R}, \quad \varepsilon>0, \quad|\mathbf{k}| \leq t^{0}
\end{array}
$$

8.2. Approximation of the smoothed bordered operator $\exp \left(-i \tau \varepsilon^{-2} \mathcal{A}(\mathbf{k})\right)$ for $|\mathbf{k}|>t^{0}$. By analogy with (7.10), we have

$$
\left\|\mathcal{R}(\mathbf{k}, \varepsilon)^{3 / 2} \widehat{P}\right\| \leq \varepsilon^{3}\left(t^{0}\right)^{-3} \leq \varepsilon\left(t^{0}\right)^{-3}, \quad 0<\varepsilon \leq 1, \quad \mathbf{k} \in \operatorname{clos} \widetilde{\Omega} \backslash \mathcal{B}\left(t^{0}\right) .
$$

Hence,

$$
\begin{aligned}
& \|(f \exp (\left.\left.-i \tau \varepsilon^{-2} \mathcal{A}(\mathbf{k})\right) f^{-1}-f_{0} \exp \left(-i \tau \varepsilon^{-2} \mathcal{A}^{0}(\mathbf{k})\right) f_{0}^{-1}\right) \mathcal{R}(\mathbf{k}, \varepsilon)^{3 / 2} \widehat{P} \|_{L_{2}(\Omega) \rightarrow L_{2}(\Omega)} \\
& \quad \leq\left(\|f\|_{L_{\infty}}\left\|f^{-1}\right\|_{L_{\infty}}+\left\|f_{0}\right\|_{L_{\infty}}\left\|f_{0}^{-1}\right\|_{L_{\infty}}\right)\left(t^{0}\right)^{-3} \varepsilon \\
& \quad \leq 2\|f\|_{L_{\infty}}\left\|f^{-1}\right\|_{L_{\infty}}\left(t^{0}\right)^{-3} \varepsilon, \quad \tau \in \mathbb{R}, \quad 0<\varepsilon \leq 1, \quad \mathbf{k} \in \operatorname{clos} \widetilde{\Omega} \backslash \mathcal{B}\left(t^{0}\right) .
\end{aligned}
$$

We have used the elementary inequalities $\left\|f_{0}\right\|_{L_{\infty}} \leq\|f\|_{L_{\infty}}$ and $\left\|f_{0}^{-1}\right\|_{L_{\infty}} \leq\left\|f^{-1}\right\|_{L_{\infty}}$. 
Now, inequalities (8.6) and (8.7) imply that

$$
\begin{array}{r}
\left\|\left(f \exp \left(-i \tau \varepsilon^{-2} \mathcal{A}(\mathbf{k})\right) f^{-1}-f_{0} \exp \left(-i \tau \varepsilon^{-2} \mathcal{A}^{0}(\mathbf{k})\right) f_{0}^{-1}\right) \mathcal{R}(\mathbf{k}, \varepsilon)^{3 / 2} \widehat{P}\right\|_{L_{2}(\Omega) \rightarrow L_{2}(\Omega)} \\
\leq\left(\|f\|_{L_{\infty}}^{2}\left\|f^{-1}\right\|_{L_{\infty}}^{2} C_{1}+2\|f\|_{L_{\infty}}\left\|f^{-1}\right\|_{L_{\infty}}\left(t^{0}\right)^{-3}+\|f\|_{L_{\infty}}^{2}\left\|f^{-1}\right\|_{L_{\infty}}^{2} C_{2}|\tau|\right) \varepsilon \\
\tau \in \mathbb{R}, \quad 0<\varepsilon \leq 1, \quad \mathbf{k} \in \operatorname{clos} \widetilde{\Omega}
\end{array}
$$

By (7.13), we have

$$
\begin{array}{r}
\left\|\left(f \exp \left(-i \tau \varepsilon^{-2} \mathcal{A}(\mathbf{k})\right) f^{-1}-f_{0} \exp \left(-i \tau \varepsilon^{-2} \mathcal{A}^{0}(\mathbf{k})\right) f_{0}^{-1}\right) \mathcal{R}(\mathbf{k}, \varepsilon)^{3 / 2}(I-\widehat{P})\right\|_{L_{2}(\Omega) \rightarrow L_{2}(\Omega)} \\
\leq 2\|f\|_{L_{\infty}}\left\|f^{-1}\right\|_{L_{\infty}} r_{0}^{-3} \varepsilon, \quad \tau \in \mathbb{R}, \quad 0<\varepsilon \leq 1, \quad \mathbf{k} \in \operatorname{clos} \widetilde{\Omega} .
\end{array}
$$

Relations (8.8) and (8.9) lead to the following result.

Theorem 8.1. For $\tau \in \mathbb{R}, 0<\varepsilon \leq 1$, and $\mathbf{k} \in \operatorname{clos} \widetilde{\Omega}$ we have

$$
\begin{aligned}
\left\|\left(f \exp \left(-i \tau \varepsilon^{-2} \mathcal{A}(\mathbf{k})\right) f^{-1}-f_{0} \exp \left(-i \tau \varepsilon^{-2} \mathcal{A}^{0}(\mathbf{k})\right) f_{0}^{-1}\right) \mathcal{R}(\mathbf{k}, \varepsilon)^{3 / 2}\right\|_{L_{2}(\Omega) \rightarrow L_{2}(\Omega)} \\
\leq\left(\mathcal{C}_{5}+\mathcal{C}_{6}|\tau|\right) \varepsilon
\end{aligned}
$$

where

$$
\begin{aligned}
& \mathcal{C}_{5}=\|f\|_{L_{\infty}}^{2}\left\|f^{-1}\right\|_{L_{\infty}}^{2} C_{1}+2\|f\|_{L_{\infty}}\left\|f^{-1}\right\|_{L_{\infty}}\left(\left(t^{0}\right)^{-3}+r_{0}^{-3}\right), \\
& \mathcal{C}_{6}=\|f\|_{L_{\infty}}^{2}\left\|f^{-1}\right\|_{L_{\infty}}^{2} C_{2} .
\end{aligned}
$$

The operator $\mathcal{R}(\mathbf{k}, \varepsilon)$ is given by (7.1), the number $t^{0}$ is defined by (5.15), and the constants $C_{1}$ and $C_{2}$ are defined by (8.3), (8.4).

8.3. Approximation of the smoothed bordered operator $\cos \left(\tau \varepsilon^{-1} \mathcal{A}(\mathbf{k})^{1 / 2}\right)$ for $|\mathbf{k}| \leq t^{0}$. The operator $\cos \left(\tau \varepsilon^{-1} \mathcal{A}(\mathbf{k})^{1 / 2}\right)$ is studied by the method described in $\S 3$ (see Subsection 3.5).

Now we indicate the constants $C_{3}$ and $C_{4}$. By (5.14), instead of the precise value of the constant (1.14), we can take

$$
C_{3}=\left(1+\beta_{2}\right) \alpha_{1}\|f\|_{L_{\infty}}^{2}\|h\|_{L_{\infty}}^{2} .
$$

The precise value of the constant (2.13) can be replaced with

$$
C_{4}=C_{1} C_{3}^{1 / 2}+C_{1} \alpha_{1}^{1 / 2}\|f\|_{L_{\infty}}\|h\|_{L_{\infty}}+\frac{1}{2} C_{2} c_{*}^{-1 / 2} .
$$

By (5.8), (8.3), (8.4), and (8.12), we obtain

$$
\begin{array}{r}
C_{4}=2 \beta_{1}\left(1+\left(1+\beta_{2}\right)^{1 / 2}\right) r_{0}^{-1} \alpha_{1} \alpha_{0}^{-1 / 2}\|h\|_{L_{\infty}}^{2}\left\|h^{-1}\right\|_{L_{\infty}}\|f\|_{L_{\infty}}^{2}\left\|f^{-1}\right\|_{L_{\infty}} \\
+\beta_{2} r_{0}^{-1} \alpha_{1}^{3 / 2} \alpha_{0}^{-1}\|h\|_{L_{\infty}}^{3}\left\|h^{-1}\right\|_{L_{\infty}}^{2}\|f\|_{L_{\infty}}^{3}\left\|f^{-1}\right\|_{L_{\infty}}^{2} .
\end{array}
$$

We use inequality (3.20) for the operator $\mathcal{A}(\mathbf{k})$. By (6.7) and (6.9), we have

$$
f_{0} \cos \left(\tau \varepsilon^{-1}\left(t^{2} f_{0} \widehat{S}(\boldsymbol{\theta}) f_{0}\right)^{1 / 2}\right) f_{0}^{-1} \widehat{P}=f_{0} \cos \left(\tau \varepsilon^{-1} \mathcal{A}^{0}(\mathbf{k})^{1 / 2}\right) f_{0}^{-1} \widehat{P},
$$

and, using (7.2), we get

$$
\begin{array}{r}
\left\|\left(f \cos \left(\tau \varepsilon^{-1} \mathcal{A}(\mathbf{k})^{1 / 2}\right) f^{-1}-f_{0} \cos \left(\tau \varepsilon^{-1} \mathcal{A}^{0}(\mathbf{k})^{1 / 2}\right) f_{0}^{-1}\right) \mathcal{R}(\mathbf{k}, \varepsilon) \widehat{P}\right\|_{L_{2}(\Omega) \rightarrow L_{2}(\Omega)} \\
\leq\|f\|_{L_{\infty}}^{2}\left\|f^{-1}\right\|_{L_{\infty}}^{2}\left(C_{1}+C_{4}|\tau|\right) \varepsilon, \quad \tau \in \mathbb{R}, \quad \varepsilon>0, \quad|\mathbf{k}| \leq t^{0} .
\end{array}
$$


8.4. Approximation of the smoothed bordered operator $\cos \left(\tau \varepsilon^{-1} \mathcal{A}(\mathbf{k})^{1 / 2}\right)$ for $|\mathbf{k}|>t^{0}$. As in (8.7), we have

$$
\begin{array}{r}
\left\|\left(f \cos \left(\tau \varepsilon^{-1} \mathcal{A}(\mathbf{k})^{1 / 2}\right) f^{-1}-f_{0} \cos \left(\tau \varepsilon^{-1} \mathcal{A}^{0}(\mathbf{k})^{1 / 2}\right) f_{0}^{-1}\right) \mathcal{R}(\mathbf{k}, \varepsilon) \widehat{P}\right\|_{L_{2}(\Omega) \rightarrow L_{2}(\Omega)} \\
\leq 2\|f\|_{L_{\infty}}\left\|f^{-1}\right\|_{L_{\infty}}\left(t^{0}\right)^{-2} \varepsilon, \quad \tau \in \mathbb{R}, \quad 0<\varepsilon \leq 1, \quad \mathbf{k} \in \operatorname{clos} \widetilde{\Omega} \backslash \mathcal{B}\left(t^{0}\right) .
\end{array}
$$

Now, (8.14) and (8.15) yield

$$
\begin{array}{r}
\left\|\left(f \cos \left(\tau \varepsilon^{-1} \mathcal{A}(\mathbf{k})^{1 / 2}\right) f^{-1}-f_{0} \cos \left(\tau \varepsilon^{-1} \mathcal{A}^{0}(\mathbf{k})^{1 / 2}\right) f_{0}^{-1}\right) \mathcal{R}(\mathbf{k}, \varepsilon) \widehat{P}\right\|_{L_{2}(\Omega) \rightarrow L_{2}(\Omega)} \\
\leq\left(\|f\|_{L_{\infty}}^{2}\left\|f^{-1}\right\|_{L_{\infty}}^{2} C_{1}+2\|f\|_{L_{\infty}}\left\|f^{-1}\right\|_{L_{\infty}}\left(t^{0}\right)^{-2}+\|f\|_{L_{\infty}}^{2}\left\|f^{-1}\right\|_{L_{\infty}}^{2} C_{4}|\tau|\right) \varepsilon \\
\tau \in \mathbb{R}, \quad 0<\varepsilon \leq 1, \quad \mathbf{k} \in \operatorname{clos} \widetilde{\Omega}
\end{array}
$$

By analogy with (8.9), we have

$$
\begin{array}{r}
\left\|\left(f \cos \left(\tau \varepsilon^{-1} \mathcal{A}(\mathbf{k})^{1 / 2}\right) f^{-1}-f_{0} \cos \left(\tau \varepsilon^{-1} \mathcal{A}^{0}(\mathbf{k})^{1 / 2}\right) f_{0}^{-1}\right) \mathcal{R}(\mathbf{k}, \varepsilon)(I-\widehat{P})\right\|_{L_{2}(\Omega) \rightarrow L_{2}(\Omega)} \\
\leq 2\|f\|_{L_{\infty}}\left\|f^{-1}\right\|_{L_{\infty}} r_{0}^{-2} \varepsilon, \quad \tau \in \mathbb{R}, \quad 0<\varepsilon \leq 1, \quad \mathbf{k} \in \operatorname{clos} \widetilde{\Omega}
\end{array}
$$

Estimates (8.16) and (8.17) yield the following statement.

Theorem 8.2. For $\tau \in \mathbb{R}, 0<\varepsilon \leq 1$, and $\mathbf{k} \in \operatorname{clos} \widetilde{\Omega}$, we have

$$
\begin{array}{r}
\left\|\left(f \cos \left(\tau \varepsilon^{-1} \mathcal{A}(\mathbf{k})^{1 / 2}\right) f^{-1}-f_{0} \cos \left(\tau \varepsilon^{-1} \mathcal{A}^{0}(\mathbf{k})^{1 / 2}\right) f_{0}^{-1}\right) \mathcal{R}(\mathbf{k}, \varepsilon)\right\|_{L_{2}(\Omega) \rightarrow L_{2}(\Omega)} \\
\leq\left(\mathcal{C}_{7}+\mathcal{C}_{8}|\tau|\right) \varepsilon
\end{array}
$$

where

$$
\begin{aligned}
& \mathcal{C}_{7}=\|f\|_{L_{\infty}}^{2}\left\|f^{-1}\right\|_{L_{\infty}}^{2} C_{1}+2\|f\|_{L_{\infty}}\left\|f^{-1}\right\|_{L_{\infty}}\left(\left(t^{0}\right)^{-2}+r_{0}^{-2}\right), \\
& \mathcal{C}_{8}=\|f\|_{L_{\infty}}^{2}\left\|f^{-1}\right\|_{L_{\infty}}^{2} C_{4} .
\end{aligned}
$$

The operator $\mathcal{R}(\mathbf{k}, \varepsilon)$ is given by (7.1), the number $t^{0}$ is defined by (5.15), and the constants $C_{1}$ and $C_{4}$ are defined by (8.3) and (8.13).

\section{§9. Approximation of the smoothed operators $\exp \left(-i \tau \varepsilon^{-2} \widehat{\mathcal{A}}\right)$ AND $\cos \left(\tau \varepsilon^{-1} \widehat{\mathcal{A}}^{1 / 2}\right)$}

9.1. Approximation of the smoothed operator $\exp \left(-i \tau \varepsilon^{-2} \widehat{\mathcal{A}}\right)$. Consider the operator $\widehat{\mathcal{A}}=b(\mathbf{D})^{*} g(\mathbf{x}) b(\mathbf{D})$ acting in $L_{2}\left(\mathbb{R}^{d} ; \mathbb{C}^{n}\right)$ (see $(6.1)$ ), and let $\widehat{\mathcal{A}}^{0}=b(\mathbf{D})^{*} g^{0} b(\mathbf{D})$ be the effective operator (6.8). We recall the notation $\mathcal{H}_{0}=-\Delta$ and put

$$
\mathcal{R}(\varepsilon):=\varepsilon^{2}\left(\mathcal{H}_{0}+\varepsilon^{2} I\right)^{-1} .
$$

Writing $\widehat{\mathcal{A}}$ in the form (5.11), we see that

$$
\exp \left(-i \tau \varepsilon^{-2} \widehat{\mathcal{A}}\right)=\mathcal{U}^{-1}\left(\int_{\tilde{\Omega}} \oplus \exp \left(-i \tau \varepsilon^{-2} \widehat{\mathcal{A}}(\mathbf{k})\right) d \mathbf{k}\right) \mathcal{U}
$$

A similar expansion is true for the operator $\exp \left(-i \tau \varepsilon^{-2} \widehat{\mathcal{A}}^{0}\right)$, and also for the difference of exponentials. The operator (9.1) expands in a direct integral of the operators (7.1):

$$
\mathcal{R}(\varepsilon)=\mathcal{U}^{-1}\left(\int_{\widetilde{\Omega}} \oplus \mathcal{R}(\mathbf{k}, \varepsilon) d \mathbf{k}\right) \mathcal{U}
$$

It follows that the operator

$$
\left(\exp \left(-i \tau \varepsilon^{-2} \widehat{\mathcal{A}}\right)-\exp \left(-i \tau \varepsilon^{-2} \widehat{\mathcal{A}}^{0}\right)\right) \mathcal{R}(\varepsilon)^{3 / 2}
$$


expands in the direct integral of the operators standing under the norm sign in (7.15). Since estimate (7.15) is uniform with respect to the parameter $\mathbf{k} \in \operatorname{clos} \widetilde{\Omega}$, Theorem 7.1 implies the following statement.

Theorem 9.1. Let $\widehat{\mathcal{A}}$ be the operator in $L_{2}\left(\mathbb{R}^{d} ; \mathbb{C}^{n}\right)$ given by $\widehat{\mathcal{A}}=b(\mathbf{D})^{*} g(\mathbf{x}) b(\mathbf{D})$, and let $\widehat{\mathcal{A}}^{0}=b(\mathbf{D})^{*} g^{0} b(\mathbf{D})$ be the effective operator. For $\tau \in \mathbb{R}$ and $0<\varepsilon \leq 1$ we have

$$
\left\|\left(\exp \left(-i \tau \varepsilon^{-2} \widehat{\mathcal{A}}\right)-\exp \left(-i \tau \varepsilon^{-2} \widehat{\mathcal{A}}^{0}\right)\right) \mathcal{R}(\varepsilon)^{3 / 2}\right\|_{L_{2}\left(\mathbb{R}^{d}\right) \rightarrow L_{2}\left(\mathbb{R}^{d}\right)} \leq\left(\mathcal{C}_{1}+\mathcal{C}_{2}|\tau|\right) \varepsilon .
$$

Here the operator $\mathcal{R}(\varepsilon)$ is defined by (9.1), and the constants $\mathcal{C}_{1}$ and $\mathcal{C}_{2}$ are defined by (7.16).

9.2. Approximation of the smoothed operator $\cos \left(\tau \varepsilon^{-1} \widehat{\mathcal{A}}^{1 / 2}\right)$. As in Subsection 9.1, the operator $\left(\cos \left(\tau \varepsilon^{-1} \widehat{\mathcal{A}}^{1 / 2}\right)-\cos \left(\tau \varepsilon^{-1}\left(\widehat{\mathcal{A}}^{0}\right)^{1 / 2}\right)\right) \mathcal{R}(\varepsilon)$ expands in the direct integral of the operators standing under the norm sign in (7.24). Therefore, Theorem 7.2 implies the following statement.

Theorem 9.2. Let $\widehat{\mathcal{A}}$ be the operator in $L_{2}\left(\mathbb{R}^{d} ; \mathbb{C}^{n}\right)$ given by $\widehat{\mathcal{A}}=b(\mathbf{D})^{*} g(\mathbf{x}) b(\mathbf{D})$, and let $\widehat{\mathcal{A}}^{0}=b(\mathbf{D})^{*} g^{0} b(\mathbf{D})$ be the effective operator. For $\tau \in \mathbb{R}$ and $0<\varepsilon \leq 1$ we have

$$
\left\|\left(\cos \left(\tau \varepsilon^{-1} \widehat{\mathcal{A}}^{1 / 2}\right)-\cos \left(\tau \varepsilon^{-1}\left(\widehat{\mathcal{A}}^{0}\right)^{1 / 2}\right)\right) \mathcal{R}(\varepsilon)\right\|_{L_{2}\left(\mathbb{R}^{d}\right) \rightarrow L_{2}\left(\mathbb{R}^{d}\right)} \leq\left(\mathcal{C}_{3}+\mathcal{C}_{4}|\tau|\right) \varepsilon .
$$

Here the operator $\mathcal{R}(\varepsilon)$ is defined by (9.1), and the constants $\mathcal{C}_{3}$ and $\mathcal{C}_{4}$ are defined by (7.25).

$$
\begin{aligned}
& \text { §10. Approximation OF THE SMOOTHED BORDERED OPERATORS } \\
& \qquad \exp \left(-i \tau \varepsilon^{-2} \mathcal{A}\right) \text { AND } \cos \left(\tau \varepsilon^{-1} \mathcal{A}^{1 / 2}\right)
\end{aligned}
$$

10.1. Approximation of the smoothed bordered operator $\exp \left(-i \tau \varepsilon^{-2} \mathcal{A}\right)$. We consider the operator $\mathcal{A}=f(\mathbf{x})^{*} b(\mathbf{D})^{*} g(\mathbf{x}) b(\mathbf{D}) f(\mathbf{x})=f(\mathbf{x})^{*} \widehat{\mathcal{A}} f(\mathbf{x})$ acting in $L_{2}\left(\mathbb{R}^{d} ; \mathbb{C}^{n}\right)$ (see (4.6)). Let $\widehat{\mathcal{A}}^{0}=b(\mathbf{D})^{*} g^{0} b(\mathbf{D})$ be the effective operator (6.8) for $\widehat{\mathcal{A}}$. Let $f_{0}$ be the constant matrix defined by (8.2), and let $\mathcal{A}^{0}=f_{0} b(\mathbf{D})^{*} g^{0} b(\mathbf{D}) f_{0}$ (see (8.5)). As in $\S 9$, we can use (5.11) to see that the operator

$$
\left(f \exp \left(-i \tau \varepsilon^{-2} \mathcal{A}\right) f^{-1}-f_{0} \exp \left(-i \tau \varepsilon^{-2} \mathcal{A}^{0}\right) f_{0}^{-1}\right) \mathcal{R}(\varepsilon)^{3 / 2}
$$

expands in the direct integral of the operators standing under the norm sign in (8.10). Therefore, Theorem 8.1 implies the following statement.

Theorem 10.1. Consider the operator in $L_{2}\left(\mathbb{R}^{d} ; \mathbb{C}^{n}\right)$ given by the formula $\mathcal{A}=$ $f(\mathbf{x})^{*} b(\mathbf{D})^{*} g(\mathbf{x}) b(\mathbf{D}) f(\mathbf{x})$. Let $\mathcal{A}^{0}=f_{0} b(\mathbf{D})^{*} g^{0} b(\mathbf{D}) f_{0}$, where $g^{0}$ is the effective matrix and $f_{0}=\left(\underline{f f^{*}}\right)^{1 / 2}$. Then for $\tau \in \mathbb{R}$ and $0<\varepsilon \leq 1$ we have

$$
\begin{aligned}
\|\left(f \exp \left(-i \tau \varepsilon^{-2} \mathcal{A}\right) f^{-1}-f_{0} \exp \left(-i \tau \varepsilon^{-2} \mathcal{A}^{0}\right) f_{0}^{-1}\right) \mathcal{R}(\varepsilon)^{3 / 2} & \|_{L_{2}\left(\mathbb{R}^{d}\right) \rightarrow L_{2}\left(\mathbb{R}^{d}\right)} \\
& \leq\left(\mathcal{C}_{5}+\mathcal{C}_{6}|\tau|\right) \varepsilon .
\end{aligned}
$$

Here the operator $\mathcal{R}(\varepsilon)$ is defined by (9.1), and the constants $\mathcal{C}_{5}$ and $\mathcal{C}_{6}$ are defined by (8.11).

10.2. Approximation of the smoothed bordered operator $\cos \left(\tau \varepsilon^{-1} \mathcal{A}^{1 / 2}\right)$. The operator

$$
\left(f \cos \left(\tau \varepsilon^{-1} \mathcal{A}^{1 / 2}\right) f^{-1}-f_{0} \cos \left(\tau \varepsilon^{-1}\left(\mathcal{A}^{0}\right)^{1 / 2}\right) f_{0}^{-1}\right) \mathcal{R}(\varepsilon)
$$

expands in the direct integral of the operators standing under the norm sign in (8.18). Therefore, Theorem 8.2 implies the following statement. 
Theorem 10.2. Consider the operator in $L_{2}\left(\mathbb{R}^{d} ; \mathbb{C}^{n}\right)$ given by the formula $\mathcal{A}=$ $f(\mathbf{x})^{*} b(\mathbf{D})^{*} g(\mathbf{x}) b(\mathbf{D}) f(\mathbf{x})$. Let $\mathcal{A}^{0}=f_{0} b(\mathbf{D})^{*} g^{0} b(\mathbf{D}) f_{0}$, where $g^{0}$ is the effective matrix and $f_{0}=\left(\underline{f f^{*}}\right)^{1 / 2}$. Then for $\tau \in \mathbb{R}$ and $0<\varepsilon \leq 1$ we have

$$
\begin{aligned}
\|\left(f \cos \left(\tau \varepsilon^{-1} \mathcal{A}^{1 / 2}\right) f^{-1}-f_{0} \cos \left(\tau \varepsilon^{-1}\left(\mathcal{A}^{0}\right)^{1 / 2}\right) f_{0}^{-1}\right) \mathcal{R}(\varepsilon) & \|_{L_{2}\left(\mathbb{R}^{d}\right) \rightarrow L_{2}\left(\mathbb{R}^{d}\right)} \\
& \leq\left(\mathcal{C}_{7}+\mathcal{C}_{8}|\tau|\right) \varepsilon .
\end{aligned}
$$

Here the operator $\mathcal{R}(\varepsilon)$ is defined by (9.1), and the constants $\mathcal{C}_{7}$ and $\mathcal{C}_{8}$ are defined by (8.19).

\section{Chapter 4. Homogenization PROBlems FOR NONSTATIONARY EQUATIONS OF MATHEMATICAL PHYSICS}

We proceed to homogenization problems in the small period limit for periodic DO's acting in $L_{2}\left(\mathbb{R}^{d} ; \mathbb{C}^{n}\right)$. We study homogenization for the Cauchy problems for the nonstationary Schrödinger equation and the hyperbolic equation. These equations involve operators $\widehat{\mathcal{A}}_{\varepsilon}$ and $\mathcal{A}_{\varepsilon}$ with rapidly oscillating coefficients (see (11.1) and (11.2)). $§ 11$ contains the statements of problems and a description of the scaling transformation. $\S 12$ is devoted to homogenization of the operator exponentials $\exp \left(-i \tau \widehat{\mathcal{A}}_{\varepsilon}\right)$ and $\exp \left(-i \tau \mathcal{A}_{\varepsilon}\right)$, and $\S 13$ is devoted to homogenization of the operator cosines $\cos \left(\tau \widehat{\mathcal{A}}_{\varepsilon}^{1 / 2}\right)$ and $\cos \left(\tau \mathcal{A}_{\varepsilon}^{1 / 2}\right)$. Here we obtain the main results of the paper in the operator terms. They are deduced from the theorems of Chapter 3 by the scaling transformation. In $\S \S 14$ and 15, using theorems given in the operator terms, we obtain the main results of the paper concerning homogenization of the Cauchy problem for the Schrödinger type equation (§14) and for the hyperbolic equation $(\S 15)$. Here various statements of problems are considered in detail.

\section{§11. Statement of problems. The scaling transformation}

11.1. The operators $\widehat{\mathcal{A}}_{\varepsilon}$ and $\mathcal{A}_{\varepsilon}$. Statement of problems. If $\psi(\mathbf{x})$ is a measurable $\Gamma$-periodic function on $\mathbb{R}^{d}$, we agree to denote $\psi^{\varepsilon}(\mathbf{x}):=\psi\left(\varepsilon^{-1} \mathbf{x}\right), \varepsilon>0$. Our main objects are the operators $\widehat{\mathcal{A}}_{\varepsilon}:=\widehat{\mathcal{A}}\left(g^{\varepsilon}\right)$ and $\mathcal{A}_{\varepsilon}:=\mathcal{A}\left(g^{\varepsilon}, f^{\varepsilon}\right), \varepsilon>0$ (see Subsection 4.1), formally given by the differential expressions

$$
\begin{aligned}
& \widehat{\mathcal{A}}_{\varepsilon}:=b(\mathbf{D})^{*} g^{\varepsilon}(\mathbf{x}) b(\mathbf{D}), \\
& \mathcal{A}_{\varepsilon}:=\left(f^{\varepsilon}(\mathbf{x})\right)^{*} b(\mathbf{D})^{*} g^{\varepsilon}(\mathbf{x}) b(\mathbf{D}) f^{\varepsilon}(\mathbf{x}) .
\end{aligned}
$$

The coefficients of the operators (11.1), (11.2) oscillate rapidly as $\varepsilon \rightarrow 0$.

Homogenization theory studies the behavior of solutions of equations of mathematical physics with rapidly oscillating coefficients. Our goal is to find approximations for small $\varepsilon$ in the $L_{2}\left(\mathbb{R}^{d} ; \mathbb{C}^{n}\right)$-norm (for a fixed $\tau$ ) for solutions of the Cauchy problem for the Schrödinger equation

$$
i \frac{\partial \mathbf{u}_{\varepsilon}}{\partial \tau}=\mathcal{A}_{\varepsilon} \mathbf{u}_{\varepsilon},
$$

and also for solutions of the Cauchy problem for the hyperbolic equation

$$
\frac{\partial^{2} \mathbf{v}_{\varepsilon}}{\partial \tau^{2}}=-\mathcal{A}_{\varepsilon} \mathbf{v}_{\varepsilon}
$$

For this purpose, we study the operator-valued functions $\exp \left(-i \tau \mathcal{A}_{\varepsilon}\right), \cos \left(\tau \mathcal{A}_{\varepsilon}^{1 / 2}\right)$, and $\mathcal{A}_{\varepsilon}^{-1 / 2} \sin \left(\tau \mathcal{A}_{\varepsilon}^{1 / 2}\right)$. The solutions of the corresponding Cauchy problems can be expressed in terms of these operator-valued functions. 
We "smooth" these operator-valued functions, obtaining approximations in the operator norm in $L_{2}\left(\mathbb{R}^{d} ; \mathbb{C}^{n}\right)$ for the corresponding smoothed operators. The required approximations are obtained in $\S \S 12$ and 13 . The results are applied to homogenization of the Cauchy problem for the Schrödinger equation (§14) and for the hyperbolic equation $(\S 15)$.

11.2. The scaling transformation. Let $T_{\varepsilon}$ be the unitary scaling transformation in $L_{2}\left(\mathbb{R}^{d} ; \mathbb{C}^{n}\right):\left(T_{\varepsilon} \mathbf{u}\right)(\mathbf{x})=\varepsilon^{d / 2} \mathbf{u}(\varepsilon \mathbf{x}), \varepsilon>0$. Then $\mathcal{A}_{\varepsilon}=\varepsilon^{-2} T_{\varepsilon}^{*} \mathcal{A} T_{\varepsilon}$. This implies the following relations for operator-valued functions of $\mathcal{A}_{\varepsilon}$ :

$$
\begin{aligned}
\exp \left(-i \tau \mathcal{A}_{\varepsilon}\right) & =T_{\varepsilon}^{*} \exp \left(-i \tau \varepsilon^{-2} \mathcal{A}\right) T_{\varepsilon}, \\
\cos \left(\tau \mathcal{A}_{\varepsilon}^{1 / 2}\right) & =T_{\varepsilon}^{*} \cos \left(\tau \varepsilon^{-1} \mathcal{A}^{1 / 2}\right) T_{\varepsilon} .
\end{aligned}
$$

Similar relations are valid for functions of $\widehat{\mathcal{A}}_{\varepsilon}$.

Applying the scaling transformation to the resolvent of the operator $\mathcal{H}_{0}=-\Delta$, we obtain

$$
\left(\mathcal{H}_{0}+I\right)^{-1}=\varepsilon^{2} T_{\varepsilon}^{*}\left(\mathcal{H}_{0}+\varepsilon^{2} I\right)^{-1} T_{\varepsilon}=T_{\varepsilon}^{*} \mathcal{R}(\varepsilon) T_{\varepsilon} .
$$

Here we have used the notation (9.1). Finally, if $\psi(\mathbf{x})$ is a periodic function in $L_{\infty}\left(\mathbb{R}^{d}\right)$, then, under the scaling transformation, the operator $\left[\psi^{\varepsilon}\right]$ of multiplication by the function $\psi^{\varepsilon}(\mathbf{x})=\psi\left(\varepsilon^{-1} \mathbf{x}\right)$ turns into the operator $[\psi]$ of multiplication by $\psi(\mathbf{x})$ :

$$
\left[\psi^{\varepsilon}\right]=T_{\varepsilon}^{*}[\psi] T_{\varepsilon} .
$$

§12. Homogenization of the operator exponential $\exp \left(-i \tau \mathcal{A}_{\varepsilon}\right)$

12.1. Approximation of the smoothed operator $\exp \left(-i \tau \widehat{\mathcal{A}}_{\varepsilon}\right)$. We start with the simpler operator (11.1). Let $\widehat{\mathcal{A}}^{0}$ be the effective operator (6.8). Combining (11.5) (for the case of $\widehat{\mathcal{A}}_{\varepsilon}$ and $\widehat{\mathcal{A}}^{0}$ ) and (11.7), we see that the operator

$$
\left(\exp \left(-i \tau \widehat{\mathcal{A}}_{\varepsilon}\right)-\exp \left(-i \tau \widehat{\mathcal{A}}^{0}\right)\right)\left(\mathcal{H}_{0}+I\right)^{-3 / 2}
$$

is unitarily equivalent to the operator under the norm sign in (9.2). Thus, Theorem 9.1 implies the following result.

Theorem 12.1. Let $\widehat{\mathcal{A}}_{\varepsilon}$ be the operator $(11.1)$, and let $\widehat{\mathcal{A}}^{0}=b(\mathbf{D})^{*} g^{0} b(\mathbf{D})$ be the corresponding effective operator. Then for $\tau \in \mathbb{R}$ and $0<\varepsilon \leq 1$ we have

$$
\left\|\left(\exp \left(-i \tau \widehat{\mathcal{A}}_{\varepsilon}\right)-\exp \left(-i \tau \widehat{\mathcal{A}}^{0}\right)\right)\left(\mathcal{H}_{0}+I\right)^{-3 / 2}\right\|_{L_{2}\left(\mathbb{R}^{d}\right) \rightarrow L_{2}\left(\mathbb{R}^{d}\right)} \leq\left(\mathcal{C}_{1}+\mathcal{C}_{2}|\tau|\right) \varepsilon
$$

The constants $\mathcal{C}_{1}$ and $\mathcal{C}_{2}$ are defined by (7.16) and depend only on $\alpha_{0}, \alpha_{1}$ in (4.2), on the norms $\|g\|_{L_{\infty}}$ and $\left\|g^{-1}\right\|_{L_{\infty}}$, and on the parameters of the lattice $\Gamma$.

Obviously,

$$
\left\|\exp \left(-i \tau \widehat{\mathcal{A}}_{\varepsilon}\right)-\exp \left(-i \tau \widehat{\mathcal{A}}^{0}\right)\right\|_{L_{2}\left(\mathbb{R}^{d}\right) \rightarrow L_{2}\left(\mathbb{R}^{d}\right)} \leq 2, \quad \tau \in \mathbb{R}, \quad \varepsilon>0 .
$$

Interpolating between (12.2) and (12.1), for $0 \leq s \leq 3$, we obtain

$$
\begin{aligned}
\left\|\left(\exp \left(-i \tau \widehat{\mathcal{A}}_{\varepsilon}\right)-\exp \left(-i \tau \widehat{\mathcal{A}}^{0}\right)\right)\left(\mathcal{H}_{0}+I\right)^{-s / 2}\right\|_{L_{2}\left(\mathbb{R}^{d}\right) \rightarrow L_{2}\left(\mathbb{R}^{d}\right)} \\
\quad \leq 2^{1-s / 3}\left(\mathcal{C}_{1}+\mathcal{C}_{2}|\tau|\right)^{s / 3} \varepsilon^{s / 3}, \quad \tau \in \mathbb{R}, \quad 0<\varepsilon \leq 1 .
\end{aligned}
$$

The operator $\left(\mathcal{H}_{0}+I\right)^{s / 2}$ is an isometric isomorphism of the Sobolev space $H^{s}\left(\mathbb{R}^{d} ; \mathbb{C}^{n}\right)$ 
onto $L_{2}\left(\mathbb{R}^{d} ; \mathbb{C}^{n}\right)$. Indeed, for $\mathbf{u} \in H^{s}\left(\mathbb{R}^{d} ; \mathbb{C}^{n}\right)$ we have

$$
\left\|\left(\mathcal{H}_{0}+I\right)^{s / 2} \mathbf{u}\right\|_{L_{2}\left(\mathbb{R}^{d}\right)}^{2}=\int_{\mathbb{R}^{d}}\left(|\boldsymbol{\xi}|^{2}+1\right)^{s}|\widehat{\mathbf{u}}(\boldsymbol{\xi})|^{2} d \boldsymbol{\xi}=\|\mathbf{u}\|_{H^{s}\left(\mathbb{R}^{d}\right)}^{2},
$$

where $\widehat{\mathbf{u}}(\boldsymbol{\xi})$ is the Fourier image of the function $\mathbf{u}(\mathbf{x})$.

Relations (12.3) and (12.4) show that

$$
\begin{array}{r}
\left\|\exp \left(-i \tau \widehat{\mathcal{A}}_{\varepsilon}\right)-\exp \left(-i \tau \widehat{\mathcal{A}}^{0}\right)\right\|_{H^{s}\left(\mathbb{R}^{d}\right) \rightarrow L_{2}\left(\mathbb{R}^{d}\right)} \leq 2^{1-s / 3}\left(\mathcal{C}_{1}+\mathcal{C}_{2}|\tau|\right)^{s / 3} \varepsilon^{s / 3}, \\
\tau \in \mathbb{R}, 0<\varepsilon \leq 1 .
\end{array}
$$

In particular, estimate (12.5) allows us to consider large values of time $\tau$. Namely, we can consider $|\tau|=O\left(\varepsilon^{-\alpha}\right)$ for $0<\alpha<1$.

We have proved the following theorem.

Theorem 12.2. Under the conditions of Theorem 12.1, if $0 \leq s \leq 3$ and $\tau \in \mathbb{R}$, $0<\varepsilon \leq 1$, then

$$
\left\|\exp \left(-i \tau \widehat{\mathcal{A}}_{\varepsilon}\right)-\exp \left(-i \tau \widehat{\mathcal{A}}^{0}\right)\right\|_{H^{s}\left(\mathbb{R}^{d}\right) \rightarrow L_{2}\left(\mathbb{R}^{d}\right)} \leq \widehat{\mathcal{C}}_{s}(\tau) \varepsilon^{s / 3},
$$

where

$$
\widehat{\mathcal{C}}_{s}(\tau)=2^{1-s / 3}\left(\mathcal{C}_{1}+\mathcal{C}_{2}|\tau|\right)^{s / 3} .
$$

In particular, for $|\tau|=\varepsilon^{-\alpha}$ and $0<\alpha<1$ we have

$$
\begin{array}{r}
\left\|\exp \left(-i \tau \widehat{\mathcal{A}}_{\varepsilon}\right)-\exp \left(-i \tau \widehat{\mathcal{A}}^{0}\right)\right\|_{H^{s}\left(\mathbb{R}^{d}\right) \rightarrow L_{2}\left(\mathbb{R}^{d}\right)} \leq \widehat{\mathcal{C}}_{s}(1) \varepsilon^{s(1-\alpha) / 3}, \\
|\tau|=\varepsilon^{-\alpha}, 0<\varepsilon \leq 1 .
\end{array}
$$

12.2. Approximation of the smoothed bordered operator $\exp \left(-i \tau \mathcal{A}_{\varepsilon}\right)$. Now we consider the general case of the operator (11.2). Let $f_{0}$ be the matrix defined by (8.2), and let $\mathcal{A}^{0}$ be the operator (8.5). Applying (11.5) (for the case of $\mathcal{A}_{\varepsilon}$ and $\mathcal{A}^{0}$ ), and also (11.7) and (11.8), we see that the operator $\left(f^{\varepsilon} e^{-i \tau \mathcal{A}_{\varepsilon}}\left(f^{\varepsilon}\right)^{-1}-f_{0} e^{-i \tau \mathcal{A}^{0}} f_{0}^{-1}\right)\left(\mathcal{H}_{0}+I\right)^{-3 / 2}$ is unitarily equivalent to the operator standing under the norm sign in (10.1). Then Theorem 10.1 implies the following result.

Theorem 12.3. Let $\mathcal{A}_{\varepsilon}$ be the operator (11.2). Let $f_{0}$ be the matrix defined by (8.2), and let $\mathcal{A}^{0}$ be the operator (8.5). Then for $\tau \in \mathbb{R}$ and $0<\varepsilon \leq 1$ we have

$$
\begin{aligned}
\|\left(f^{\varepsilon} \exp \left(-i \tau \mathcal{A}_{\varepsilon}\right)\left(f^{\varepsilon}\right)^{-1}-f_{0} \exp \left(-i \tau \mathcal{A}^{0}\right) f_{0}^{-1}\right)\left(\mathcal{H}_{0}+I\right)^{-3 / 2} & \|_{L_{2}\left(\mathbb{R}^{d}\right) \rightarrow L_{2}\left(\mathbb{R}^{d}\right)} \\
& \leq\left(\mathcal{C}_{5}+\mathcal{C}_{6}|\tau|\right) \varepsilon .
\end{aligned}
$$

The constants $\mathcal{C}_{5}$ and $\mathcal{C}_{6}$ are defined by (8.11) and depend only on $\alpha_{0}, \alpha_{1}$ in (4.2), on the norms $\|g\|_{L_{\infty}},\left\|g^{-1}\right\|_{L_{\infty}},\|f\|_{L_{\infty}},\left\|f^{-1}\right\|_{L_{\infty}}$, and on the parameters of the lattice $\Gamma$.

Obviously,

$$
\begin{aligned}
& \left\|f^{\varepsilon} \exp \left(-i \tau \mathcal{A}_{\varepsilon}\right)\left(f^{\varepsilon}\right)^{-1}-f_{0} \exp \left(-i \tau \mathcal{A}^{0}\right) f_{0}^{-1}\right\|_{L_{2}\left(\mathbb{R}^{d}\right) \rightarrow L_{2}\left(\mathbb{R}^{d}\right)} \\
& \quad \leq 2\|f\|_{L_{\infty}}\left\|f^{-1}\right\|_{L_{\infty}}, \quad \tau \in \mathbb{R}, \quad \varepsilon>0 .
\end{aligned}
$$

Interpolating between (12.10) and (12.9) and using (12.4), for $0 \leq s \leq 3$, we obtain

$$
\begin{aligned}
& \left\|f^{\varepsilon} \exp \left(-i \tau \mathcal{A}_{\varepsilon}\right)\left(f^{\varepsilon}\right)^{-1}-f_{0} \exp \left(-i \tau \mathcal{A}^{0}\right) f_{0}^{-1}\right\|_{H^{s}\left(\mathbb{R}^{d}\right) \rightarrow L_{2}\left(\mathbb{R}^{d}\right)} \\
& \quad \leq 2^{1-s / 3}\|f\|_{L_{\infty}}^{1-s / 3}\left\|f^{-1}\right\|_{L_{\infty}}^{1-s / 3}\left(\mathcal{C}_{5}+\mathcal{C}_{6}|\tau|\right)^{s / 3} \varepsilon^{s / 3}, \quad \tau \in \mathbb{R}, \quad 0<\varepsilon \leq 1 .
\end{aligned}
$$

We have proved the following theorem. 
Theorem 12.4. Under the conditions of Theorem 12.3, if $0 \leq s \leq 3$ and $\tau \in \mathbb{R}$, $0<\varepsilon \leq 1$, then

$$
\left\|f^{\varepsilon} \exp \left(-i \tau \mathcal{A}_{\varepsilon}\right)\left(f^{\varepsilon}\right)^{-1}-f_{0} \exp \left(-i \tau \mathcal{A}^{0}\right) f_{0}^{-1}\right\|_{H^{s}\left(\mathbb{R}^{d}\right) \rightarrow L_{2}\left(\mathbb{R}^{d}\right)} \leq \mathcal{C}_{s}(\tau) \varepsilon^{s / 3},
$$

where

$$
\mathcal{C}_{s}(\tau)=2^{1-s / 3}\|f\|_{L_{\infty}}^{1-s / 3}\left\|f^{-1}\right\|_{L_{\infty}}^{1-s / 3}\left(\mathcal{C}_{5}+\mathcal{C}_{6}|\tau|\right)^{s / 3} .
$$

In particular, for $|\tau|=\varepsilon^{-\alpha}$ and $0<\alpha<1$ we have

$$
\begin{array}{r}
\left\|f^{\varepsilon} \exp \left(-i \tau \mathcal{A}_{\varepsilon}\right)\left(f^{\varepsilon}\right)^{-1}-f_{0} \exp \left(-i \tau \mathcal{A}^{0}\right) f_{0}^{-1}\right\|_{H^{s}\left(\mathbb{R}^{d}\right) \rightarrow L_{2}\left(\mathbb{R}^{d}\right)} \leq \mathcal{C}_{s}(1) \varepsilon^{s(1-\alpha) / 3}, \\
|\tau|=\varepsilon^{-\alpha}, 0<\varepsilon \leq 1 .
\end{array}
$$

§13. Homogenization of the operators $\cos \left(\tau \mathcal{A}_{\varepsilon}^{1 / 2}\right)$ And $\mathcal{A}_{\varepsilon}^{-1 / 2} \sin \left(\tau \mathcal{A}_{\varepsilon}^{1 / 2}\right)$

13.1. Approximation of the smoothed operators $\cos \left(\tau \widehat{\mathcal{A}}_{\varepsilon}^{1 / 2}\right)$ and $\widehat{\mathcal{A}}_{\varepsilon}^{-1 / 2} \sin \left(\tau \widehat{\mathcal{A}}_{\varepsilon}^{1 / 2}\right)$. Let $\widehat{\mathcal{A}}_{\varepsilon}$ be the operator (11.1). Let $\widehat{\mathcal{A}}^{0}$ be the effective operator (6.8). Combining (11.6) (for the case of $\widehat{\mathcal{A}}_{\varepsilon}$ and $\left.\widehat{\mathcal{A}}^{0}\right)$ and (11.7), we see that the operator $\left(\cos \left(\tau \widehat{\mathcal{A}}_{\varepsilon}^{1 / 2}\right)-\right.$ $\left.\cos \left(\tau\left(\widehat{\mathcal{A}}^{0}\right)^{1 / 2}\right)\right)\left(\mathcal{H}_{0}+I\right)^{-1}$ is unitarily equivalent to the operator under the norm sign in (9.3). Then Theorem 9.2 implies the following result.

Theorem 13.1. Let $\widehat{\mathcal{A}}_{\varepsilon}$ be the operator $(11.1)$, and let $\widehat{\mathcal{A}}^{0}=b(\mathbf{D})^{*} g^{0} b(\mathbf{D})$ be the corresponding effective operator. Then for $\tau \in \mathbb{R}$ and $0<\varepsilon \leq 1$ we have

$$
\left\|\left(\cos \left(\tau \widehat{\mathcal{A}}_{\varepsilon}^{1 / 2}\right)-\cos \left(\tau\left(\widehat{\mathcal{A}}^{0}\right)^{1 / 2}\right)\right)\left(\mathcal{H}_{0}+I\right)^{-1}\right\|_{L_{2}\left(\mathbb{R}^{d}\right) \rightarrow L_{2}\left(\mathbb{R}^{d}\right)} \leq\left(\mathcal{C}_{3}+\mathcal{C}_{4}|\tau|\right) \varepsilon .
$$

The constants $\mathcal{C}_{3}$ and $\mathcal{C}_{4}$ are defined by (7.25) and depend only on $\alpha_{0}, \alpha_{1}$ in (4.2), on the norms $\|g\|_{L_{\infty}}$ and $\left\|g^{-1}\right\|_{L_{\infty}}$, and on the parameters of the lattice $\Gamma$.

Obviously,

$$
\left\|\cos \left(\tau \widehat{\mathcal{A}}_{\varepsilon}^{1 / 2}\right)-\cos \left(\tau\left(\widehat{\mathcal{A}}^{0}\right)^{1 / 2}\right)\right\|_{L_{2}\left(\mathbb{R}^{d}\right) \rightarrow L_{2}\left(\mathbb{R}^{d}\right)} \leq 2, \quad \tau \in \mathbb{R}, \quad \varepsilon>0 .
$$

Interpolating between (13.2) and (13.1) and using (12.4), for $0 \leq s \leq 2$, we obtain

$$
\begin{array}{r}
\left\|\cos \left(\tau \widehat{\mathcal{A}}_{\varepsilon}^{1 / 2}\right)-\cos \left(\tau\left(\widehat{\mathcal{A}}^{0}\right)^{1 / 2}\right)\right\|_{H^{s}\left(\mathbb{R}^{d}\right) \rightarrow L_{2}\left(\mathbb{R}^{d}\right)} \leq 2^{1-s / 2}\left(\mathcal{C}_{3}+\mathcal{C}_{4}|\tau|\right)^{s / 2} \varepsilon^{s / 2} \\
\tau \in \mathbb{R}, \quad 0<\varepsilon \leq 1
\end{array}
$$

Since

$$
\widehat{\mathcal{A}}_{\varepsilon}^{-1 / 2} \sin \left(\tau \widehat{\mathcal{A}}_{\varepsilon}^{1 / 2}\right)=\int_{0}^{\tau} \cos \left(\tilde{\tau} \widehat{\mathcal{A}}_{\varepsilon}^{1 / 2}\right) d \tilde{\tau},
$$

and a similar identity is valid for $\widehat{\mathcal{A}}^{0}$, from (13.3) we can deduce that

$$
\begin{aligned}
& \left\|\widehat{\mathcal{A}}_{\varepsilon}^{-1 / 2} \sin \left(\tau \widehat{\mathcal{A}}_{\varepsilon}^{1 / 2}\right)-\left(\widehat{\mathcal{A}}^{0}\right)^{-1 / 2} \sin \left(\tau\left(\widehat{\mathcal{A}}^{0}\right)^{1 / 2}\right)\right\|_{H^{s}\left(\mathbb{R}^{d}\right) \rightarrow L_{2}\left(\mathbb{R}^{d}\right)} \\
& \quad \leq 2^{1-s / 2}(1+s / 2)^{-1} \mathcal{C}_{4}^{-1}\left(\mathcal{C}_{3}+\mathcal{C}_{4}|\tau|\right)^{1+s / 2} \varepsilon^{s / 2}, \quad \tau \in \mathbb{R}, \quad 0<\varepsilon \leq 1 .
\end{aligned}
$$

Note that for large values of $|\tau|$ the coefficient of $\varepsilon^{s / 2}$ in (13.3) is $O\left(|\tau|^{s / 2}\right)$, and in (13.4) the coefficient of $\varepsilon^{s / 2}$ is $O\left(|\tau|^{1+s / 2}\right)$.

We have proved the following theorem.

Theorem 13.2. Under the conditions of Theorem 13.1, if $0 \leq s \leq 2$ and $\tau \in \mathbb{R}$, $0<\varepsilon \leq 1$, then

$$
\begin{aligned}
& \left\|\cos \left(\tau \widehat{\mathcal{A}}_{\varepsilon}^{1 / 2}\right)-\cos \left(\tau\left(\widehat{\mathcal{A}}^{0}\right)^{1 / 2}\right)\right\|_{H^{s}\left(\mathbb{R}^{d}\right) \rightarrow L_{2}\left(\mathbb{R}^{d}\right)} \leq \widehat{\mathfrak{C}}_{s}(\tau) \varepsilon^{s / 2}, \\
& \left\|\widehat{\mathcal{A}}_{\varepsilon}^{-1 / 2} \sin \left(\tau \widehat{\mathcal{A}}_{\varepsilon}^{1 / 2}\right)-\left(\widehat{\mathcal{A}}^{0}\right)^{-1 / 2} \sin \left(\tau\left(\widehat{\mathcal{A}}^{0}\right)^{1 / 2}\right)\right\|_{H^{s}\left(\mathbb{R}^{d}\right) \rightarrow L_{2}\left(\mathbb{R}^{d}\right)} \leq \widehat{\mathfrak{C}}_{s}^{\prime}(\tau) \varepsilon^{s / 2},
\end{aligned}
$$


where

$$
\widehat{\mathfrak{C}}_{s}(\tau)=2^{1-s / 2}\left(\mathcal{C}_{3}+\mathcal{C}_{4}|\tau|\right)^{s / 2}, \quad \widehat{\mathfrak{C}}_{s}^{\prime}(\tau)=\widehat{\mathfrak{C}}_{s}(\tau)(1+s / 2)^{-1}\left(\mathcal{C}_{3} \mathcal{C}_{4}^{-1}+|\tau|\right) .
$$

In particular, for $|\tau|=\varepsilon^{-\alpha}$ we have

$$
\begin{array}{r}
\left\|\cos \left(\tau \widehat{\mathcal{A}}_{\varepsilon}^{1 / 2}\right)-\cos \left(\tau\left(\widehat{\mathcal{A}}^{0}\right)^{1 / 2}\right)\right\|_{H^{s}\left(\mathbb{R}^{d}\right) \rightarrow L_{2}\left(\mathbb{R}^{d}\right)} \leq \widehat{\mathfrak{C}}_{s}(1) \varepsilon^{s(1-\alpha) / 2} \\
|\tau|=\varepsilon^{-\alpha}, 0<\alpha<1,0<\varepsilon \leq 1, \\
\left\|\widehat{\mathcal{A}}_{\varepsilon}^{-1 / 2} \sin \left(\tau \widehat{\mathcal{A}}_{\varepsilon}^{1 / 2}\right)-\left(\widehat{\mathcal{A}}^{0}\right)^{-1 / 2} \sin \left(\tau\left(\widehat{\mathcal{A}}^{0}\right)^{1 / 2}\right)\right\|_{H^{s}\left(\mathbb{R}^{d}\right) \rightarrow L_{2}\left(\mathbb{R}^{d}\right)} \leq \widehat{\mathfrak{C}}_{s}^{\prime}(1) \varepsilon^{s(1-\alpha) / 2-\alpha} \\
|\tau|=\varepsilon^{-\alpha}, \quad 0<\alpha<s(s+2)^{-1}, 0<\varepsilon \leq 1 .
\end{array}
$$

13.2. Approximation for the smoothed bordered operators $\cos \left(\tau \mathcal{A}_{\varepsilon}^{1 / 2}\right)$ and $\mathcal{A}_{\varepsilon}^{-1 / 2} \sin \left(\tau \mathcal{A}_{\varepsilon}^{1 / 2}\right)$. Now we consider the general case of the operators (11.2). Let $f_{0}$ be the matrix defined by (8.2), and let $\mathcal{A}^{0}$ be the operator (8.5). Applying (11.6) (for the case of $\mathcal{A}_{\varepsilon}$ and $\mathcal{A}^{0}$ ) and also (11.7) and (11.8), we see that the operator

$$
\left(f^{\varepsilon} \cos \left(\tau \mathcal{A}_{\varepsilon}^{1 / 2}\right)\left(f^{\varepsilon}\right)^{-1}-f_{0} \cos \left(\tau\left(\mathcal{A}^{0}\right)^{1 / 2}\right) f_{0}^{-1}\right)\left(\mathcal{H}_{0}+I\right)^{-1}
$$

is unitarily equivalent to the operator standing under the norm sign in (10.2). Then Theorem 10.2 implies the following result.

Theorem 13.3. Let $\mathcal{A}_{\varepsilon}$ be the operator (11.2), and let $\mathcal{A}^{0}$ be the operator (8.5). Then for $\tau \in \mathbb{R}$ and $0<\varepsilon \leq 1$ we have

$$
\begin{aligned}
\left\|\left(f^{\varepsilon} \cos \left(\tau \mathcal{A}_{\varepsilon}^{1 / 2}\right)\left(f^{\varepsilon}\right)^{-1}-f_{0} \cos \left(\tau\left(\mathcal{A}^{0}\right)^{1 / 2}\right) f_{0}^{-1}\right)\left(\mathcal{H}_{0}+I\right)^{-1}\right\|_{L_{2}\left(\mathbb{R}^{d}\right) \rightarrow L_{2}\left(\mathbb{R}^{d}\right)} \\
\leq\left(\mathcal{C}_{7}+\mathcal{C}_{8}|\tau|\right) \varepsilon .
\end{aligned}
$$

The constants $\mathcal{C}_{7}$ and $\mathcal{C}_{8}$ are defined by (8.19) and depend only on $\alpha_{0}, \alpha_{1}$ in (4.2), on the norms $\|g\|_{L_{\infty}},\left\|g^{-1}\right\|_{L_{\infty}},\|f\|_{L_{\infty}},\left\|f^{-1}\right\|_{L_{\infty}}$, and on the parameters of the lattice $\Gamma$.

Obviously,

$$
\begin{array}{r}
\left\|f^{\varepsilon} \cos \left(\tau \mathcal{A}_{\varepsilon}^{1 / 2}\right)\left(f^{\varepsilon}\right)^{-1}-f_{0} \cos \left(\tau\left(\mathcal{A}^{0}\right)^{1 / 2}\right) f_{0}^{-1}\right\|_{L_{2}\left(\mathbb{R}^{d}\right) \rightarrow L_{2}\left(\mathbb{R}^{d}\right)} \leq 2\|f\|_{L_{\infty}}\left\|f^{-1}\right\|_{L_{\infty}}, \\
\tau \in \mathbb{R}, \quad \varepsilon>0 .
\end{array}
$$

Interpolating between (13.11) and (13.10) and using (12.4), for $0 \leq s \leq 2$, we obtain

$$
\begin{aligned}
& \left\|f^{\varepsilon} \cos \left(\tau \mathcal{A}_{\varepsilon}^{1 / 2}\right)\left(f^{\varepsilon}\right)^{-1}-f_{0} \cos \left(\tau\left(\mathcal{A}^{0}\right)^{1 / 2}\right) f_{0}^{-1}\right\|_{H^{s}\left(\mathbb{R}^{d}\right) \rightarrow L_{2}\left(\mathbb{R}^{d}\right)} \\
& \quad \leq 2^{1-s / 2}\|f\|_{L_{\infty}}^{1-s / 2}\left\|f^{-1}\right\|_{L_{\infty}}^{1-s / 2}\left(\mathcal{C}_{7}+\mathcal{C}_{8}|\tau|\right)^{s / 2} \varepsilon^{s / 2}, \quad \tau \in \mathbb{R}, \quad 0<\varepsilon \leq 1 .
\end{aligned}
$$

We have proved the following theorem.

Theorem 13.4. Under the conditions of Theorem 13.3, if $0 \leq s \leq 2$ and $\tau \in \mathbb{R}$, $0<\varepsilon \leq 1$, then

$$
\begin{aligned}
& \left\|f^{\varepsilon} \cos \left(\tau \mathcal{A}_{\varepsilon}^{1 / 2}\right)\left(f^{\varepsilon}\right)^{-1}-f_{0} \cos \left(\tau\left(\mathcal{A}^{0}\right)^{1 / 2}\right) f_{0}^{-1}\right\|_{H^{s}\left(\mathbb{R}^{d}\right) \rightarrow L_{2}\left(\mathbb{R}^{d}\right)} \leq \mathfrak{C}_{s}(\tau) \varepsilon^{s / 2}, \\
& \left\|f^{\varepsilon} \mathcal{A}_{\varepsilon}^{-1 / 2} \sin \left(\tau \mathcal{A}_{\varepsilon}^{1 / 2}\right)\left(f^{\varepsilon}\right)^{-1}-f_{0}\left(\mathcal{A}^{0}\right)^{-1 / 2} \sin \left(\tau\left(\mathcal{A}^{0}\right)^{1 / 2}\right) f_{0}^{-1}\right\|_{H^{s}\left(\mathbb{R}^{d}\right) \rightarrow L_{2}\left(\mathbb{R}^{d}\right)} \leq \mathfrak{C}_{s}^{\prime}(\tau) \varepsilon^{s / 2},
\end{aligned}
$$

where

$$
\begin{aligned}
\mathfrak{C}_{s}(\tau) & =2^{1-s / 2}\|f\|_{L_{\infty}}^{1-s / 2}\left\|f^{-1}\right\|_{L_{\infty}}^{1-s / 2}\left(\mathcal{C}_{7}+\mathcal{C}_{8}|\tau|\right)^{s / 2} \\
\mathfrak{C}_{s}^{\prime}(\tau) & =\mathfrak{C}_{s}(\tau)(1+s / 2)^{-1}\left(\mathcal{C}_{7} \mathcal{C}_{8}^{-1}+|\tau|\right) .
\end{aligned}
$$


In particular, for $|\tau|=\varepsilon^{-\alpha}$ we have

$$
\begin{array}{r}
\left\|f^{\varepsilon} \cos \left(\tau \mathcal{A}_{\varepsilon}^{1 / 2}\right)\left(f^{\varepsilon}\right)^{-1}-f_{0} \cos \left(\tau\left(\mathcal{A}^{0}\right)^{1 / 2}\right) f_{0}^{-1}\right\|_{H^{s}\left(\mathbb{R}^{d}\right) \rightarrow L_{2}\left(\mathbb{R}^{d}\right)} \leq \mathfrak{C}_{s}(1) \varepsilon^{s(1-\alpha) / 2}, \\
|\tau|=\varepsilon^{-\alpha}, \quad 0<\alpha<1, \quad 0<\varepsilon \leq 1, \\
\left\|f^{\varepsilon} \mathcal{A}_{\varepsilon}^{-1 / 2} \sin \left(\tau \mathcal{A}_{\varepsilon}^{1 / 2}\right)\left(f^{\varepsilon}\right)^{-1}-f_{0}\left(\mathcal{A}^{0}\right)^{-1 / 2} \sin \left(\tau\left(\mathcal{A}^{0}\right)^{1 / 2}\right) f_{0}^{-1}\right\|_{H^{s}\left(\mathbb{R}^{d}\right) \rightarrow L_{2}\left(\mathbb{R}^{d}\right)} \\
\leq \mathfrak{C}_{s}^{\prime}(1) \varepsilon^{s(1-\alpha) / 2-\alpha}, \quad|\tau|=\varepsilon^{-\alpha}, \quad 0<\alpha<s(s+2)^{-1}, \quad 0<\varepsilon \leq 1 .
\end{array}
$$

\section{§14. Homogenization of the Cauchy problem FOR THE SCHRÖDINGER-TYPE EQUATION}

14.1. The Cauchy problem for equation (11.3) with the operator $\widehat{\mathcal{A}}_{\varepsilon}$. Let $\widehat{\mathcal{A}}_{\varepsilon}$ be the operator (11.1). We consider the following Cauchy problem for a function $\mathbf{u}_{\varepsilon}(\mathbf{x}, \tau)$, $\mathbf{x} \in \mathbb{R}^{d}, \tau \in \mathbb{R}:$

$$
i \frac{\partial \mathbf{u}_{\varepsilon}(\mathbf{x}, \tau)}{\partial \tau}=b(\mathbf{D})^{*} g^{\varepsilon}(\mathbf{x}) b(\mathbf{D}) \mathbf{u}_{\varepsilon}(\mathbf{x}, \tau), \quad \mathbf{u}_{\varepsilon}(\mathbf{x}, 0)=\phi(\mathbf{x}),
$$

where $\phi \in L_{2}\left(\mathbb{R}^{d} ; \mathbb{C}^{n}\right)$ is a given function. The solution of class $L_{2}\left(\mathbb{R}^{d} ; \mathbb{C}^{n}\right)$ is given by $\mathbf{u}_{\varepsilon}=\exp \left(-i \tau \widehat{\mathcal{A}}_{\varepsilon}\right) \phi$. Let $\mathbf{u}_{0}(\mathbf{x}, \tau)$ be the solution of the "homogenized" Cauchy problem

$$
i \frac{\partial \mathbf{u}_{0}(\mathbf{x}, \tau)}{\partial \tau}=b(\mathbf{D})^{*} g^{0} b(\mathbf{D}) \mathbf{u}_{0}(\mathbf{x}, \tau), \quad \mathbf{u}_{0}(\mathbf{x}, 0)=\phi(\mathbf{x}),
$$

where $g^{0}$ is the effective matrix. Then $\mathbf{u}_{0}=\exp \left(-i \tau \widehat{\mathcal{A}}^{0}\right) \phi$.

Theorem 12.2 implies the following result.

Theorem 14.1. Let $\mathbf{u}_{\varepsilon}$ be the solution of (14.1), and let $\mathbf{u}_{0}$ be the solution of (14.2).

$1^{\circ}$. If $\phi \in H^{s}\left(\mathbb{R}^{d} ; \mathbb{C}^{n}\right), 0 \leq s \leq 3$, then for $\tau \in \mathbb{R}$ and $0<\varepsilon \leq 1$ we have

$$
\left\|\mathbf{u}_{\varepsilon}(\cdot, \tau)-\mathbf{u}_{0}(\cdot, \tau)\right\|_{L_{2}\left(\mathbb{R}^{d}\right)} \leq \varepsilon^{s / 3} \widehat{\mathcal{C}}_{s}(\tau)\|\phi\|_{H^{s}\left(\mathbb{R}^{d}\right)} .
$$

In particular, for $\tau= \pm \varepsilon^{-\alpha}, 0<\alpha<1$, we have

$$
\left\|\mathbf{u}_{\varepsilon}\left(\cdot, \pm \varepsilon^{-\alpha}\right)-\mathbf{u}_{0}\left(\cdot, \pm \varepsilon^{-\alpha}\right)\right\|_{L_{2}\left(\mathbb{R}^{d}\right)} \leq \varepsilon^{s(1-\alpha) / 3} \widehat{\mathcal{C}}_{s}(1)\|\phi\|_{H^{s}\left(\mathbb{R}^{d}\right)}, \quad 0<\varepsilon \leq 1 .
$$

The constant $\widehat{\mathcal{C}}_{s}(\tau)$ is defined by $(12.7)$.

$2^{\circ}$. If $\phi \in L_{2}\left(\mathbb{R}^{d} ; \mathbb{C}^{n}\right)$, then

$$
\begin{aligned}
\lim _{\varepsilon \rightarrow 0}\left\|\mathbf{u}_{\varepsilon}(\cdot, \tau)-\mathbf{u}_{0}(\cdot, \tau)\right\|_{L_{2}\left(\mathbb{R}^{d}\right)}=0, & \tau \in \mathbb{R} ; \\
\lim _{\varepsilon \rightarrow 0}\left\|\mathbf{u}_{\varepsilon}\left(\cdot, \pm \varepsilon^{-\alpha}\right)-\mathbf{u}_{0}\left(\cdot, \pm \varepsilon^{-\alpha}\right)\right\|_{L_{2}\left(\mathbb{R}^{d}\right)}=0, & 0<\alpha<1 .
\end{aligned}
$$

Statement $2^{\circ}$ follows directly from statement $1^{\circ}$ and the Banach-Steinhaus theorem.

14.2. The nonhomogeneous Cauchy problem with the operator $\widehat{\mathcal{A}}_{\varepsilon}$. Now we consider a more general problem,

$$
i \frac{\partial \mathbf{u}_{\varepsilon}(\mathbf{x}, \tau)}{\partial \tau}=b(\mathbf{D})^{*} g^{\varepsilon}(\mathbf{x}) b(\mathbf{D}) \mathbf{u}_{\varepsilon}(\mathbf{x}, \tau)+\mathbf{F}(\mathbf{x}, \tau), \quad \mathbf{u}_{\varepsilon}(\mathbf{x}, 0)=\phi(\mathbf{x})
$$

where $\phi \in L_{2}\left(\mathbb{R}^{d} ; \mathbb{C}^{n}\right)$ and $\mathbf{F} \in L_{1, \text { loc }}\left(\mathbb{R} ; L_{2}\left(\mathbb{R}^{d} ; \mathbb{C}^{n}\right)\right)$. The solution of this problem can be represented as

$$
\mathbf{u}_{\varepsilon}(\cdot, \tau)=\exp \left(-i \tau \widehat{\mathcal{A}}_{\varepsilon}\right) \phi-i \int_{0}^{\tau} \exp \left(-i(\tau-\widetilde{\tau}) \widehat{\mathcal{A}}_{\varepsilon}\right) \mathbf{F}(\cdot, \widetilde{\tau}) d \widetilde{\tau}
$$

Let $\mathbf{u}_{0}(\mathbf{x}, \tau)$ be the solution of the homogenized problem

$$
i \frac{\partial \mathbf{u}_{0}(\mathbf{x}, \tau)}{\partial \tau}=b(\mathbf{D})^{*} g^{0} b(\mathbf{D}) \mathbf{u}_{0}(\mathbf{x}, \tau)+\mathbf{F}(\mathbf{x}, \tau), \quad \mathbf{u}_{0}(\mathbf{x}, 0)=\phi(\mathbf{x}) .
$$


Then

$$
\mathbf{u}_{0}(\cdot, \tau)=\exp \left(-i \tau \widehat{\mathcal{A}}^{0}\right) \boldsymbol{\phi}-i \int_{0}^{\tau} \exp \left(-i(\tau-\widetilde{\tau}) \widehat{\mathcal{A}}^{0}\right) \mathbf{F}(\cdot, \widetilde{\tau}) d \widetilde{\tau}
$$

Now, if $\phi \in H^{s}\left(\mathbb{R}^{d} ; \mathbb{C}^{n}\right)$ and $\mathbf{F} \in L_{1, \text { loc }}\left(\mathbb{R} ; H^{s}\left(\mathbb{R}^{d} ; \mathbb{C}^{n}\right)\right)$ for some $s \in[0,3]$, then estimate (12.6) implies

$$
\begin{aligned}
& \left\|\mathbf{u}_{\varepsilon}(\cdot, \tau)-\mathbf{u}_{0}(\cdot, \tau)\right\|_{L_{2}\left(\mathbb{R}^{d}\right)} \\
& \quad \leq \varepsilon^{s / 3} \widehat{\mathcal{C}}_{s}(\tau)\|\phi\|_{H^{s}\left(\mathbb{R}^{d}\right)}+\varepsilon^{s / 3}\left|\int_{0}^{\tau} \widehat{\mathcal{C}}_{s}(\tau-\widetilde{\tau})\|\mathbf{F}(\cdot, \widetilde{\tau})\|_{H^{s}\left(\mathbb{R}^{d}\right)} d \widetilde{\tau}\right| \\
& \quad \leq \varepsilon^{s / 3} \widehat{\mathcal{C}}_{s}(\tau)\left(\|\phi\|_{H^{s}\left(\mathbb{R}^{d}\right)}+\|\mathbf{F}\|_{L_{1}\left((0, \tau) ; H^{s}\left(\mathbb{R}^{d}\right)\right)}\right), \quad \tau \in \mathbb{R}, \quad 0<\varepsilon \leq 1 .
\end{aligned}
$$

If, moreover, it is known that $\mathbf{F} \in L_{p}\left(\mathbb{R}_{ \pm} ; H^{s}\left(\mathbb{R}^{d} ; \mathbb{C}^{n}\right)\right)$ for some $p \in[1, \infty]$, then, by (12.8), for $\tau= \pm \varepsilon^{-\alpha}$ we have

$$
\begin{aligned}
&\left\|\mathbf{u}_{\varepsilon}\left(\cdot, \pm \varepsilon^{-\alpha}\right)-\mathbf{u}_{0}\left(\cdot, \pm \varepsilon^{-\alpha}\right)\right\|_{L_{2}\left(\mathbb{R}^{d}\right)} \\
& \leq \varepsilon^{s(1-\alpha) / 3} \widehat{\mathcal{C}}_{s}(1)\left(\|\phi\|_{H^{s}\left(\mathbb{R}^{d}\right)}+\left|\int_{0}^{ \pm \varepsilon^{-\alpha}}\|\mathbf{F}(\cdot, \widetilde{\tau})\|_{H^{s}\left(\mathbb{R}^{d}\right)} d \widetilde{\tau}\right|\right) \\
& \leq \varepsilon^{s(1-\alpha) / 3} \widehat{\mathcal{C}}_{s}(1)\left(\|\phi\|_{H^{s}\left(\mathbb{R}^{d}\right)}+\varepsilon^{-\alpha / p^{\prime}}\|\mathbf{F}\|_{L_{p}\left(\mathbb{R}_{ \pm} ; H^{s}\left(\mathbb{R}^{d}\right)\right)}\right), \\
& 0<\alpha<\frac{s}{s+3 / p^{\prime}}, \quad 0<\varepsilon \leq 1 .
\end{aligned}
$$

Here $p^{-1}+\left(p^{\prime}\right)^{-1}=1$.

Now, suppose that it is only known that $\phi \in L_{2}\left(\mathbb{R}^{d} ; \mathbb{C}^{n}\right)$ and $\mathbf{F} \in L_{1, \text { loc }}\left(\mathbb{R} ; L_{2}\left(\mathbb{R}^{d} ; \mathbb{C}^{n}\right)\right)$. By the Banach-Steinhaus theorem, the obvious estimate

$$
\left\|\mathbf{u}_{\varepsilon}(\cdot, \tau)-\mathbf{u}_{0}(\cdot, \tau)\right\|_{L_{2}\left(\mathbb{R}^{d}\right)} \leq 2\|\phi\|_{L_{2}\left(\mathbb{R}^{d}\right)}+2\|\mathbf{F}\|_{L_{1}\left((0, \tau) ; L_{2}\left(\mathbb{R}^{d}\right)\right)}
$$

and (14.6) imply that $\mathbf{u}_{\varepsilon}(\cdot, \tau)$ tends to $\mathbf{u}_{0}(\cdot, \tau)$ strongly in $L_{2}\left(\mathbb{R}^{d} ; \mathbb{C}^{n}\right)$ for any fixed $\tau \in \mathbb{R}$. If, moreover, it is known that $\mathbf{F} \in L_{1}\left(\mathbb{R}_{ \pm} ; L_{2}\left(\mathbb{R}^{d} ; \mathbb{C}^{n}\right)\right)$, then for $\tau= \pm \varepsilon^{-\alpha}, 0<\alpha<1$, we obviously have

$$
\left\|\mathbf{u}_{\varepsilon}\left(\cdot, \pm \varepsilon^{-\alpha}\right)-\mathbf{u}_{0}\left(\cdot, \pm \varepsilon^{-\alpha}\right)\right\|_{L_{2}\left(\mathbb{R}^{d}\right)} \leq 2\|\phi\|_{L_{2}\left(\mathbb{R}^{d}\right)}+2\|\mathbf{F}\|_{L_{1}\left(\mathbb{R}_{ \pm} ; L_{2}\left(\mathbb{R}^{d}\right)\right)} .
$$

By the Banach-Steinhaus theorem, from (14.8) and (14.7) (with $p=1$ ) it follows that the left-hand side of (14.8) tends to zero.

Thus, we have proved the following theorem.

Theorem 14.2. Let $\mathbf{u}_{\varepsilon}$ be the solution of problem (14.4), and $\mathbf{u}_{0}$ the solution of problem (14.5).

$1^{\circ}$. If $\phi \in H^{s}\left(\mathbb{R}^{d} ; \mathbb{C}^{n}\right)$ and $\mathbf{F} \in L_{1, \text { loc }}\left(\mathbb{R} ; H^{s}\left(\mathbb{R}^{d} ; \mathbb{C}^{n}\right)\right)$ for some $s \in[0,3]$, then for $\tau \in \mathbb{R}$ and $0<\varepsilon \leq 1$ we have

$$
\left\|\mathbf{u}_{\varepsilon}(\cdot, \tau)-\mathbf{u}_{0}(\cdot, \tau)\right\|_{L_{2}\left(\mathbb{R}^{d}\right)} \leq \varepsilon^{s / 3} \widehat{\mathcal{C}}_{s}(\tau)\left(\|\phi\|_{H^{s}\left(\mathbb{R}^{d}\right)}+\|\mathbf{F}\|_{L_{1}\left((0, \tau) ; H^{s}\left(\mathbb{R}^{d}\right)\right)}\right) .
$$

If, moreover, $\mathbf{F} \in L_{p}\left(\mathbb{R}_{ \pm} ; H^{s}\left(\mathbb{R}^{d} ; \mathbb{C}^{n}\right)\right)($ where $1 \leq p \leq \infty)$, then for $\tau= \pm \varepsilon^{-\alpha}, 0<\alpha<$ $s\left(s+3 / p^{\prime}\right)^{-1}$, and $0<\varepsilon \leq 1$, we have

$$
\begin{aligned}
\| \mathbf{u}_{\varepsilon}\left(\cdot, \pm \varepsilon^{-\alpha}\right) & -\mathbf{u}_{0}\left(\cdot, \pm \varepsilon^{-\alpha}\right) \|_{L_{2}\left(\mathbb{R}^{d}\right)} \\
& \leq \varepsilon^{s(1-\alpha) / 3} \widehat{\mathcal{C}}_{s}(1)\left(\|\boldsymbol{\phi}\|_{H^{s}\left(\mathbb{R}^{d}\right)}+\varepsilon^{-\alpha / p^{\prime}}\|\mathbf{F}\|_{L_{p}\left(\mathbb{R}_{ \pm} ; H^{s}\left(\mathbb{R}^{d}\right)\right)}\right) .
\end{aligned}
$$

The constant $\widehat{\mathcal{C}}_{s}(\tau)$ is defined by $(12.7)$.

$2^{\circ}$. If $\boldsymbol{\phi} \in L_{2}\left(\mathbb{R}^{d} ; \mathbb{C}^{n}\right)$ and $\mathbf{F} \in L_{1, \mathrm{loc}}\left(\mathbb{R} ; L_{2}\left(\mathbb{R}^{d} ; \mathbb{C}^{n}\right)\right)$, then

$$
\lim _{\varepsilon \rightarrow 0}\left\|\mathbf{u}_{\varepsilon}(\cdot, \tau)-\mathbf{u}_{0}(\cdot, \tau)\right\|_{L_{2}\left(\mathbb{R}^{d}\right)}=0, \quad \tau \in \mathbb{R} .
$$


If, moreover, $\mathbf{F} \in L_{1}\left(\mathbb{R}_{ \pm} ; L_{2}\left(\mathbb{R}^{d} ; \mathbb{C}^{n}\right)\right)$, then

$$
\lim _{\varepsilon \rightarrow 0}\left\|\mathbf{u}_{\varepsilon}\left(\cdot, \pm \varepsilon^{-\alpha}\right)-\mathbf{u}_{0}\left(\cdot, \pm \varepsilon^{-\alpha}\right)\right\|_{L_{2}\left(\mathbb{R}^{d}\right)}=0, \quad 0<\alpha<1
$$

14.3. The Cauchy problem for equation (11.3) with the operator $\mathcal{A}_{\varepsilon}$. Let $\mathcal{A}_{\varepsilon}$ be the operator (11.2). We consider the following Cauchy problem:

$$
i \frac{\partial \mathbf{u}_{\varepsilon}(\mathbf{x}, \tau)}{\partial \tau}=\left(f^{\varepsilon}(\mathbf{x})\right)^{*} b(\mathbf{D})^{*} g^{\varepsilon}(\mathbf{x}) b(\mathbf{D}) f^{\varepsilon}(\mathbf{x}) \mathbf{u}_{\varepsilon}(\mathbf{x}, \tau), \quad f^{\varepsilon}(\mathbf{x}) \mathbf{u}_{\varepsilon}(\mathbf{x}, 0)=\phi(\mathbf{x}),
$$

where $\phi \in L_{2}\left(\mathbb{R}^{d} ; \mathbb{C}^{n}\right)$ is a given function. The solution $\mathbf{u}_{\varepsilon}$ of problem (14.9) is represented as $\mathbf{u}_{\varepsilon}=\exp \left(-i \tau \mathcal{A}_{\varepsilon}\right)\left(f^{\varepsilon}\right)^{-1} \boldsymbol{\phi}$. Let $\mathbf{u}_{0}(\mathbf{x}, \tau)$ be the solution of the "homogenized" Cauchy problem

$$
i \frac{\partial \mathbf{u}_{0}(\mathbf{x}, \tau)}{\partial \tau}=f_{0} b(\mathbf{D})^{*} g^{0} b(\mathbf{D}) f_{0} \mathbf{u}_{0}(\mathbf{x}, \tau), \quad f_{0} \mathbf{u}_{0}(\mathbf{x}, 0)=\phi(\mathbf{x}) .
$$

Here $g^{0}$ is the effective matrix, and $f_{0}$ is the matrix defined by (8.2). Then $\mathbf{u}_{0}=$ $\exp \left(-i \tau \mathcal{A}^{0}\right)\left(f_{0}\right)^{-1} \phi$.

Theorem 12.4 directly implies the following result.

Theorem 14.3. Let $\mathbf{u}_{\varepsilon}$ be the solution of (14.9), and $\mathbf{u}_{0}$ the solution of (14.10).

$1^{\circ}$. If $\phi \in H^{s}\left(\mathbb{R}^{d} ; \mathbb{C}^{n}\right), 0 \leq s \leq 3$, then for $\tau \in \mathbb{R}$ and $0<\varepsilon \leq 1$ we have

$$
\left\|f^{\varepsilon} \mathbf{u}_{\varepsilon}(\cdot, \tau)-f_{0} \mathbf{u}_{0}(\cdot, \tau)\right\|_{L_{2}\left(\mathbb{R}^{d}\right)} \leq \varepsilon^{s / 3} \mathcal{C}_{s}(\tau)\|\phi\|_{H^{s}\left(\mathbb{R}^{d}\right)} .
$$

In particular, for $\tau= \pm \varepsilon^{-\alpha}, 0<\alpha<1$, we have

$$
\left\|f^{\varepsilon} \mathbf{u}_{\varepsilon}\left(\cdot, \pm \varepsilon^{-\alpha}\right)-f_{0} \mathbf{u}_{0}\left(\cdot, \pm \varepsilon^{-\alpha}\right)\right\|_{L_{2}\left(\mathbb{R}^{d}\right)} \leq \varepsilon^{s(1-\alpha) / 3} \mathcal{C}_{s}(1)\|\phi\|_{H^{s}\left(\mathbb{R}^{d}\right)}, \quad 0<\varepsilon \leq 1 .
$$

The constant $\mathcal{C}_{s}(\tau)$ is defined by (12.12).

$2^{\circ}$. If $\phi \in L_{2}\left(\mathbb{R}^{d} ; \mathbb{C}^{n}\right)$, then

$$
\begin{array}{ll}
\lim _{\varepsilon \rightarrow 0}\left\|f^{\varepsilon} \mathbf{u}_{\varepsilon}(\cdot, \tau)-f_{0} \mathbf{u}_{0}(\cdot, \tau)\right\|_{L_{2}\left(\mathbb{R}^{d}\right)}=0, & \tau \in \mathbb{R} ; \\
\lim _{\varepsilon \rightarrow 0}\left\|f^{\varepsilon} \mathbf{u}_{\varepsilon}\left(\cdot, \pm \varepsilon^{-\alpha}\right)-f_{0} \mathbf{u}_{0}\left(\cdot, \pm \varepsilon^{-\alpha}\right)\right\|_{L_{2}\left(\mathbb{R}^{d}\right)}=0, & 0<\alpha<1 .
\end{array}
$$

Note that problem (14.9) admits a different formulation. By the substitution $f^{\varepsilon} \mathbf{u}_{\varepsilon}=$ $\mathbf{w}_{\varepsilon}$, problem (14.9) turns into the following Cauchy problem for a function $\mathbf{w}_{\varepsilon}(\mathbf{x}, \tau)$ :

$$
i Q^{\varepsilon}(\mathbf{x}) \frac{\partial \mathbf{w}_{\varepsilon}(\mathbf{x}, \tau)}{\partial \tau}=b(\mathbf{D})^{*} g^{\varepsilon}(\mathbf{x}) b(\mathbf{D}) \mathbf{w}_{\varepsilon}(\mathbf{x}, \tau), \quad \mathbf{w}_{\varepsilon}(\mathbf{x}, 0)=\phi(\mathbf{x}) .
$$

Here $Q^{\varepsilon}(\mathbf{x})=\left(f^{\varepsilon}(\mathbf{x})\left(f^{\varepsilon}(\mathbf{x})\right)^{*}\right)^{-1}$. By the substitution $f_{0} \mathbf{u}_{0}=\mathbf{w}_{0}$, the homogenized problem (14.10) turns into the problem

$$
i \bar{Q} \frac{\partial \mathbf{w}_{0}(\mathbf{x}, \tau)}{\partial \tau}=b(\mathbf{D})^{*} g^{0} b(\mathbf{D}) \mathbf{w}_{0}(\mathbf{x}, \tau), \quad \mathbf{w}_{0}(\mathbf{x}, 0)=\phi(\mathbf{x}) .
$$

Here $\bar{Q}$ is the mean value of the matrix $Q(\mathbf{x})$ over $\Omega$. We have used relation (8.2).

Formula (14.12) can be viewed as the initial statement of the problem. Then we assume that $Q(\mathbf{x})$ is a $\Gamma$-periodic $(n \times n)$-matrix-valued function on $\mathbb{R}^{d}$ such that

$$
Q(\mathbf{x})>0 ; \quad Q, Q^{-1} \in L_{\infty} .
$$

Theorem 14.3 admits the following equivalent formulation.

Theorem 14.4. Let $\mathbf{w}_{\varepsilon}$ be the solution of problem (14.12), and $\mathbf{w}_{0}$ the solution of problem (14.13).

$1^{\circ}$. If $\phi \in H^{s}\left(\mathbb{R}^{d} ; \mathbb{C}^{n}\right), 0 \leq s \leq 3$, then for $\tau \in \mathbb{R}$ and $0<\varepsilon \leq 1$ we have

$$
\left\|\mathbf{w}_{\varepsilon}(\cdot, \tau)-\mathbf{w}_{0}(\cdot, \tau)\right\|_{L_{2}\left(\mathbb{R}^{d}\right)} \leq \varepsilon^{s / 3} \mathcal{C}_{s}(\tau)\|\phi\|_{H^{s}\left(\mathbb{R}^{d}\right)} .
$$


In particular, for $\tau= \pm \varepsilon^{-\alpha}, 0<\alpha<1$, we have

$$
\left\|\mathbf{w}_{\varepsilon}\left(\cdot, \pm \varepsilon^{-\alpha}\right)-\mathbf{w}_{0}\left(\cdot, \pm \varepsilon^{-\alpha}\right)\right\|_{L_{2}\left(\mathbb{R}^{d}\right)} \leq \varepsilon^{s(1-\alpha) / 3} \mathcal{C}_{s}(1)\|\phi\|_{H^{s}\left(\mathbb{R}^{d}\right)}, \quad 0<\varepsilon \leq 1 .
$$

The constant $\mathcal{C}_{s}(\tau)$ is defined by (12.12).

$2^{\circ}$. If $\phi \in L_{2}\left(\mathbb{R}^{d} ; \mathbb{C}^{n}\right)$, then

$$
\begin{array}{ll}
\lim _{\varepsilon \rightarrow 0}\left\|\mathbf{w}_{\varepsilon}(\cdot, \tau)-\mathbf{w}_{0}(\cdot, \tau)\right\|_{L_{2}\left(\mathbb{R}^{d}\right)}=0, & \tau \in \mathbb{R} ; \\
\lim _{\varepsilon \rightarrow 0}\left\|\mathbf{w}_{\varepsilon}\left(\cdot, \pm \varepsilon^{-\alpha}\right)-\mathbf{w}_{0}\left(\cdot, \pm \varepsilon^{-\alpha}\right)\right\|_{L_{2}\left(\mathbb{R}^{d}\right)}=0, & 0<\alpha<1 .
\end{array}
$$

14.4. The nonhomogeneous Cauchy problem with the operator $\mathcal{A}_{\varepsilon}$. Now we consider a problem more general than (14.9):

$$
\begin{aligned}
i \frac{\partial \mathbf{u}_{\varepsilon}(\mathbf{x}, \tau)}{\partial \tau} & =\left(f^{\varepsilon}(\mathbf{x})\right)^{*} b(\mathbf{D})^{*} g^{\varepsilon}(\mathbf{x}) b(\mathbf{D}) f^{\varepsilon}(\mathbf{x}) \mathbf{u}_{\varepsilon}(\mathbf{x}, \tau)+\left(f^{\varepsilon}(\mathbf{x})\right)^{-1} \mathbf{F}(\mathbf{x}, \tau), \\
f^{\varepsilon}(\mathbf{x}) \mathbf{u}_{\varepsilon}(\mathbf{x}, 0) & =\phi(\mathbf{x}),
\end{aligned}
$$

where $\phi \in L_{2}\left(\mathbb{R}^{d} ; \mathbb{C}^{n}\right)$ and $\mathbf{F} \in L_{1, \text { loc }}\left(\mathbb{R} ; L_{2}\left(\mathbb{R}^{d} ; \mathbb{C}^{n}\right)\right)$. The solution of this problem is represented as

$$
\mathbf{u}_{\varepsilon}(\cdot, \tau)=\exp \left(-i \tau \mathcal{A}_{\varepsilon}\right)\left(f^{\varepsilon}\right)^{-1} \boldsymbol{\phi}-i \int_{0}^{\tau} \exp \left(-i(\tau-\widetilde{\tau}) \mathcal{A}_{\varepsilon}\right)\left(f^{\varepsilon}\right)^{-1} \mathbf{F}(\cdot, \widetilde{\tau}) d \widetilde{\tau} .
$$

Let $\mathbf{u}_{0}(\mathbf{x}, \tau)$ be the solution of the homogenized problem

$$
i \frac{\partial \mathbf{u}_{0}(\mathbf{x}, \tau)}{\partial \tau}=f_{0} b(\mathbf{D})^{*} g^{0} b(\mathbf{D}) f_{0} \mathbf{u}_{0}(\mathbf{x}, \tau)+f_{0}^{-1} \mathbf{F}(\mathbf{x}, \tau), \quad f_{0} \mathbf{u}_{0}(\mathbf{x}, 0)=\phi(\mathbf{x}) .
$$

Then

$$
\mathbf{u}_{0}(\cdot, \tau)=\exp \left(-i \tau \mathcal{A}^{0}\right) f_{0}^{-1} \phi-i \int_{0}^{\tau} \exp \left(-i(\tau-\widetilde{\tau}) \mathcal{A}^{0}\right) f_{0}^{-1} \mathbf{F}(\cdot, \widetilde{\tau}) d \widetilde{\tau} .
$$

Now, if $\phi \in H^{s}\left(\mathbb{R}^{d} ; \mathbb{C}^{n}\right)$ and $\mathbf{F} \in L_{1, \text { loc }}\left(\mathbb{R} ; H^{s}\left(\mathbb{R}^{d} ; \mathbb{C}^{n}\right)\right)$ for some $s \in[0,3]$, then estimate (12.11) implies

$$
\begin{aligned}
& \left\|f^{\varepsilon} \mathbf{u}_{\varepsilon}(\cdot, \tau)-f_{0} \mathbf{u}_{0}(\cdot, \tau)\right\|_{L_{2}\left(\mathbb{R}^{d}\right)} \\
& \quad \leq \varepsilon^{s / 3} \mathcal{C}_{s}(\tau)\|\phi\|_{H^{s}\left(\mathbb{R}^{d}\right)}+\varepsilon^{s / 3}\left|\int_{0}^{\tau} \mathcal{C}_{s}(\tau-\widetilde{\tau})\|\mathbf{F}(\cdot, \widetilde{\tau})\|_{H^{s}\left(\mathbb{R}^{d}\right)} d \widetilde{\tau}\right| \\
& \quad \leq \varepsilon^{s / 3} \mathcal{C}_{s}(\tau)\left(\|\phi\|_{H^{s}\left(\mathbb{R}^{d}\right)}+\|\mathbf{F}\|_{L_{1}\left((0, \tau) ; H^{s}\left(\mathbb{R}^{d}\right)\right)}\right), \quad \tau \in \mathbb{R}, \quad 0<\varepsilon \leq 1 .
\end{aligned}
$$

In the case where $|\tau|=\varepsilon^{-\alpha}$ we should use estimate (12.13).

As a result, we obtain the following theorem (cf. the proof of Theorem 14.2).

Theorem 14.5. Let $\mathbf{u}_{\varepsilon}$ be the solution of problem (14.15), and $\mathbf{u}_{0}$ the solution of problem (14.16).

$1^{\circ}$. If $\phi \in H^{s}\left(\mathbb{R}^{d} ; \mathbb{C}^{n}\right)$ and $\mathbf{F} \in L_{1, \text { loc }}\left(\mathbb{R} ; H^{s}\left(\mathbb{R}^{d} ; \mathbb{C}^{n}\right)\right)$ for some $s \in[0,3]$, then for $\tau \in \mathbb{R}$ and $0<\varepsilon \leq 1$ we have

$$
\left\|f^{\varepsilon} \mathbf{u}_{\varepsilon}(\cdot, \tau)-f_{0} \mathbf{u}_{0}(\cdot, \tau)\right\|_{L_{2}\left(\mathbb{R}^{d}\right)} \leq \varepsilon^{s / 3} \mathcal{C}_{s}(\tau)\left(\|\phi\|_{H^{s}\left(\mathbb{R}^{d}\right)}+\|\mathbf{F}\|_{L_{1}\left((0, \tau) ; H^{s}\left(\mathbb{R}^{d}\right)\right)}\right) .
$$

If, moreover, $\mathbf{F} \in L_{p}\left(\mathbb{R}_{ \pm} ; H^{s}\left(\mathbb{R}^{d} ; \mathbb{C}^{n}\right)\right)($ where $1 \leq p \leq \infty)$, then for $\tau= \pm \varepsilon^{-\alpha}, 0<\alpha<$ $s\left(s+3 / p^{\prime}\right)^{-1}$, and $0<\varepsilon \leq 1$, we have

$$
\begin{aligned}
\| f^{\varepsilon} \mathbf{u}_{\varepsilon}\left(\cdot, \pm \varepsilon^{-\alpha}\right) & -f_{0} \mathbf{u}_{0}\left(\cdot, \pm \varepsilon^{-\alpha}\right) \|_{L_{2}\left(\mathbb{R}^{d}\right)} \\
& \leq \varepsilon^{s(1-\alpha) / 3} \mathcal{C}_{s}(1)\left(\|\phi\|_{H^{s}\left(\mathbb{R}^{d}\right)}+\varepsilon^{-\alpha / p^{\prime}}\|\mathbf{F}\|_{L_{p}\left(\mathbb{R}_{ \pm} ; H^{s}\left(\mathbb{R}^{d}\right)\right)}\right) .
\end{aligned}
$$

The constant $\mathcal{C}_{s}(\tau)$ is defined by (12.12).

$2^{\circ}$. If $\boldsymbol{\phi} \in L_{2}\left(\mathbb{R}^{d} ; \mathbb{C}^{n}\right)$ and $\mathbf{F} \in L_{1, \mathrm{loc}}\left(\mathbb{R} ; L_{2}\left(\mathbb{R}^{d} ; \mathbb{C}^{n}\right)\right)$, then

$$
\lim _{\varepsilon \rightarrow 0}\left\|f^{\varepsilon} \mathbf{u}_{\varepsilon}(\cdot, \tau)-f_{0} \mathbf{u}_{0}(\cdot, \tau)\right\|_{L_{2}\left(\mathbb{R}^{d}\right)}=0, \quad \tau \in \mathbb{R} .
$$


If, moreover, $\mathbf{F} \in L_{1}\left(\mathbb{R}_{ \pm} ; L_{2}\left(\mathbb{R}^{d} ; \mathbb{C}^{n}\right)\right)$, then

$$
\lim _{\varepsilon \rightarrow 0}\left\|f^{\varepsilon} \mathbf{u}_{\varepsilon}\left(\cdot, \pm \varepsilon^{-\alpha}\right)-f_{0} \mathbf{u}_{0}\left(\cdot, \pm \varepsilon^{-\alpha}\right)\right\|_{L_{2}\left(\mathbb{R}^{d}\right)}=0, \quad 0<\alpha<1 .
$$

Problem (14.15) admits a different formulation. By the substitution $f^{\varepsilon} \mathbf{u}_{\varepsilon}=\mathbf{w}_{\varepsilon}$, (14.15) turns into the following Cauchy problem for a function $\mathbf{w}_{\varepsilon}(\mathbf{x}, \tau)$ :

$$
i Q^{\varepsilon}(\mathbf{x}) \frac{\partial \mathbf{w}_{\varepsilon}(\mathbf{x}, \tau)}{\partial \tau}=b(\mathbf{D})^{*} g^{\varepsilon}(\mathbf{x}) b(\mathbf{D}) \mathbf{w}_{\varepsilon}(\mathbf{x}, \tau)+Q^{\varepsilon}(\mathbf{x}) \mathbf{F}(\mathbf{x}, \tau), \quad \mathbf{w}_{\varepsilon}(\mathbf{x}, 0)=\boldsymbol{\phi}(\mathbf{x}),
$$

where $Q^{\varepsilon}(\mathbf{x})=\left(f^{\varepsilon}(\mathbf{x})\left(f^{\varepsilon}(\mathbf{x})\right)^{*}\right)^{-1}$. By the substitution $f_{0} \mathbf{u}_{0}=\mathbf{w}_{0}$, the homogenized problem (14.16) turns into

$$
i \bar{Q} \frac{\partial \mathbf{w}_{0}(\mathbf{x}, \tau)}{\partial \tau}=b(\mathbf{D})^{*} g^{0} b(\mathbf{D}) \mathbf{w}_{0}(\mathbf{x}, \tau)+\bar{Q} \mathbf{F}(\mathbf{x}, \tau), \quad \mathbf{w}_{0}(\mathbf{x}, 0)=\phi(\mathbf{x}) .
$$

If (14.17) is regarded as the initial statement of the problem, then it is assumed that $Q(\mathbf{x})$ is a $\Gamma$-periodic matrix-valued function satisfying condition (14.14).

Theorem 14.5 admits the following equivalent formulation.

Theorem 14.6. Let $\mathbf{w}_{\varepsilon}$ be the solution of problem (14.17), and $\mathbf{w}_{0}$ the solution of problem (14.18).

$1^{\circ}$. If $\phi \in H^{s}\left(\mathbb{R}^{d} ; \mathbb{C}^{n}\right)$ and $\mathbf{F} \in L_{1, \text { loc }}\left(\mathbb{R} ; H^{s}\left(\mathbb{R}^{d} ; \mathbb{C}^{n}\right)\right)$ for some $s \in[0,3]$, then for $\tau \in \mathbb{R}$ and $0<\varepsilon \leq 1$ we have

$$
\left\|\mathbf{w}_{\varepsilon}(\cdot, \tau)-\mathbf{w}_{0}(\cdot, \tau)\right\|_{L_{2}\left(\mathbb{R}^{d}\right)} \leq \varepsilon^{s / 3} \mathcal{C}_{s}(\tau)\left(\|\phi\|_{H^{s}\left(\mathbb{R}^{d}\right)}+\|\mathbf{F}\|_{L_{1}\left((0, \tau) ; H^{s}\left(\mathbb{R}^{d}\right)\right)}\right) .
$$

If, moreover, $\mathbf{F} \in L_{p}\left(\mathbb{R}_{ \pm} ; H^{s}\left(\mathbb{R}^{d} ; \mathbb{C}^{n}\right)\right)($ where $1 \leq p \leq \infty)$, then for $\tau= \pm \varepsilon^{-\alpha}, 0<\alpha<$ $s\left(s+3 / p^{\prime}\right)^{-1}$, and $0<\varepsilon \leq 1$, we have

$$
\begin{aligned}
\| \mathbf{w}_{\varepsilon}\left(\cdot, \pm \varepsilon^{-\alpha}\right) & -\mathbf{w}_{0}\left(\cdot, \pm \varepsilon^{-\alpha}\right) \|_{L_{2}\left(\mathbb{R}^{d}\right)} \\
& \leq \varepsilon^{s(1-\alpha) / 3} \mathcal{C}_{s}(1)\left(\|\phi\|_{H^{s}\left(\mathbb{R}^{d}\right)}+\varepsilon^{-\alpha / p^{\prime}}\|\mathbf{F}\|_{L_{p}\left(\mathbb{R}_{ \pm} ; H^{s}\left(\mathbb{R}^{d}\right)\right)}\right) .
\end{aligned}
$$

The constant $\mathcal{C}_{s}(\tau)$ is defined by (12.12).

$2^{\circ}$. If $\boldsymbol{\phi} \in L_{2}\left(\mathbb{R}^{d} ; \mathbb{C}^{n}\right)$ and $\mathbf{F} \in L_{1, \mathrm{loc}}\left(\mathbb{R} ; L_{2}\left(\mathbb{R}^{d} ; \mathbb{C}^{n}\right)\right)$, then

$$
\lim _{\varepsilon \rightarrow 0}\left\|\mathbf{w}_{\varepsilon}(\cdot, \tau)-\mathbf{w}_{0}(\cdot, \tau)\right\|_{L_{2}\left(\mathbb{R}^{d}\right)}=0, \quad \tau \in \mathbb{R} .
$$

If, moreover, $\mathbf{F} \in L_{1}\left(\mathbb{R}_{ \pm} ; L_{2}\left(\mathbb{R}^{d} ; \mathbb{C}^{n}\right)\right)$, then

$$
\lim _{\varepsilon \rightarrow 0}\left\|\mathbf{w}_{\varepsilon}\left(\cdot, \pm \varepsilon^{-\alpha}\right)-\mathbf{w}_{0}\left(\cdot, \pm \varepsilon^{-\alpha}\right)\right\|_{L_{2}\left(\mathbb{R}^{d}\right)}=0, \quad 0<\alpha<1 .
$$

§15. Homogenization of the Cauchy problem for the hyperbolic equation

15.1. The Cauchy problem for equation (11.4) with the operator $\widehat{\mathcal{A}}_{\varepsilon}$. Let $\widehat{\mathcal{A}}_{\varepsilon}$ be the operator (11.1). Consider the following Cauchy problem for a function $\mathbf{v}_{\varepsilon}(\mathbf{x}, \tau)$, $\mathbf{x} \in \mathbb{R}^{d}, \tau \in \mathbb{R}:$

$$
\begin{aligned}
\frac{\partial^{2} \mathbf{v}_{\varepsilon}(\mathbf{x}, \tau)}{\partial \tau^{2}} & =-b(\mathbf{D})^{*} g^{\varepsilon}(\mathbf{x}) b(\mathbf{D}) \mathbf{v}_{\varepsilon}(\mathbf{x}, \tau), \\
\mathbf{v}_{\varepsilon}(\mathbf{x}, 0) & =\boldsymbol{\phi}(\mathbf{x}), \quad \frac{\partial \mathbf{v}_{\varepsilon}}{\partial \tau}(\mathbf{x}, 0)=\boldsymbol{\psi}(\mathbf{x}),
\end{aligned}
$$

where $\boldsymbol{\phi}, \boldsymbol{\psi} \in L_{2}\left(\mathbb{R}^{d} ; \mathbb{C}^{n}\right)$ are given functions. The solution of class $L_{2}\left(\mathbb{R}^{d} ; \mathbb{C}^{n}\right)$ is given by the formula

$$
\mathbf{v}_{\varepsilon}=\cos \left(\tau \widehat{\mathcal{A}}_{\varepsilon}^{1 / 2}\right) \phi+\widehat{\mathcal{A}}_{\varepsilon}^{-1 / 2} \sin \left(\tau \widehat{\mathcal{A}}_{\varepsilon}^{1 / 2}\right) \boldsymbol{\psi} .
$$

Let $\mathbf{v}_{0}(\mathbf{x}, \tau)$ be the solution of the "homogenized" Cauchy problem

$$
\frac{\partial^{2} \mathbf{v}_{0}(\mathbf{x}, \tau)}{\partial \tau^{2}}=-b(\mathbf{D})^{*} g^{0} b(\mathbf{D}) \mathbf{v}_{0}(\mathbf{x}, \tau), \quad \mathbf{v}_{0}(\mathbf{x}, 0)=\phi(\mathbf{x}), \quad \frac{\partial \mathbf{v}_{0}}{\partial \tau}(\mathbf{x}, 0)=\boldsymbol{\psi}(\mathbf{x})
$$


where $g^{0}$ is the effective matrix. Then

$$
\mathbf{v}_{0}=\cos \left(\tau\left(\widehat{\mathcal{A}}^{0}\right)^{1 / 2}\right) \boldsymbol{\phi}+\left(\widehat{\mathcal{A}}^{0}\right)^{-1 / 2} \sin \left(\tau\left(\widehat{\mathcal{A}}^{0}\right)^{1 / 2}\right) \boldsymbol{\psi} .
$$

Theorem 13.2 implies the following result.

Theorem 15.1. Let $\mathbf{v}_{\varepsilon}$ be the solution of problem (15.1), and $\mathbf{v}_{0}$ the solution of problem (15.2).

$1^{\circ}$. If $\boldsymbol{\phi}, \boldsymbol{\psi} \in H^{s}\left(\mathbb{R}^{d} ; \mathbb{C}^{n}\right), 0 \leq s \leq 2$, then for $\tau \in \mathbb{R}$ and $0<\varepsilon \leq 1$ we have

$$
\left\|\mathbf{v}_{\varepsilon}(\cdot, \tau)-\mathbf{v}_{0}(\cdot, \tau)\right\|_{L_{2}\left(\mathbb{R}^{d}\right)} \leq \varepsilon^{s / 2}\left(\widehat{\mathfrak{C}}_{s}(\tau)\|\phi\|_{H^{s}\left(\mathbb{R}^{d}\right)}+\widehat{\mathfrak{C}}_{s}^{\prime}(\tau)\|\boldsymbol{\psi}\|_{H^{s}\left(\mathbb{R}^{d}\right)}\right) .
$$

In particular, for $\tau= \pm \varepsilon^{-\alpha}$ and $0<\varepsilon \leq 1$ we have

$$
\begin{aligned}
\| \mathbf{v}_{\varepsilon}\left(\cdot, \pm \varepsilon^{-\alpha}\right) & -\mathbf{v}_{0}\left(\cdot, \pm \varepsilon^{-\alpha}\right) \|_{L_{2}\left(\mathbb{R}^{d}\right)} \\
& \leq \varepsilon^{s(1-\alpha) / 2}\left(\widehat{\mathfrak{C}}_{s}(1)\|\boldsymbol{\phi}\|_{H^{s}\left(\mathbb{R}^{d}\right)}+\varepsilon^{-\alpha} \widehat{\mathfrak{C}}_{s}^{\prime}(1)\|\boldsymbol{\psi}\|_{H^{s}\left(\mathbb{R}^{d}\right)}\right) .
\end{aligned}
$$

Here $0<\alpha<s(s+2)^{-1}$ if $\boldsymbol{\psi} \neq 0$, and $0<\alpha<1$ if $\boldsymbol{\psi}=0$. The constants $\widehat{\mathfrak{C}}_{s}(\tau)$ and $\widehat{\mathfrak{C}}_{s}^{\prime}(\tau)$ are defined by $(13.7)$.

$2^{\circ}$. If $\boldsymbol{\phi}, \boldsymbol{\psi} \in L_{2}\left(\mathbb{R}^{d} ; \mathbb{C}^{n}\right)$, then

$$
\lim _{\varepsilon \rightarrow 0}\left\|\mathbf{v}_{\varepsilon}(\cdot, \tau)-\mathbf{v}_{0}(\cdot, \tau)\right\|_{L_{2}\left(\mathbb{R}^{d}\right)}=0, \quad \tau \in \mathbb{R} .
$$

$3^{\circ}$. If $\phi \in L_{2}\left(\mathbb{R}^{d} ; \mathbb{C}^{n}\right)$ and $\boldsymbol{\psi}=0$, then

$$
\lim _{\varepsilon \rightarrow 0}\left\|\mathbf{v}_{\varepsilon}\left(\cdot, \pm \varepsilon^{-\alpha}\right)-\mathbf{v}_{0}\left(\cdot, \pm \varepsilon^{-\alpha}\right)\right\|_{L_{2}\left(\mathbb{R}^{d}\right)}=0, \quad 0<\alpha<1 .
$$

By the Banach-Steinhaus theorem, statement $2^{\circ}$ follows directly from (15.3) and the obvious estimate

$$
\left\|\mathbf{v}_{\varepsilon}(\cdot, \tau)-\mathbf{v}_{0}(\cdot, \tau)\right\|_{L_{2}\left(\mathbb{R}^{d}\right)} \leq 2\|\phi\|_{L_{2}\left(\mathbb{R}^{d}\right)}+2|\tau|\|\boldsymbol{\psi}\|_{L_{2}\left(\mathbb{R}^{d}\right)} .
$$

Again by the Banach-Steinhaus theorem, statement $3^{\circ}$ follows from (15.4) (with $\boldsymbol{\psi}=0$ and $0<\alpha<1$ ) and the obvious estimate

$$
\left\|\mathbf{v}_{\varepsilon}\left(\cdot, \pm \varepsilon^{-\alpha}\right)-\mathbf{v}_{0}\left(\cdot, \pm \varepsilon^{-\alpha}\right)\right\|_{L_{2}\left(\mathbb{R}^{d}\right)} \leq 2\|\phi\|_{L_{2}\left(\mathbb{R}^{d}\right)} \text { for } \boldsymbol{\psi}=0 .
$$

15.2. The nonhomogeneous Cauchy problem with the operator $\widehat{\mathcal{A}}_{\varepsilon}$. Now we consider the more general problem

$$
\begin{aligned}
\frac{\partial^{2} \mathbf{v}_{\varepsilon}(\mathbf{x}, \tau)}{\partial \tau^{2}} & =-b(\mathbf{D})^{*} g^{\varepsilon}(\mathbf{x}) b(\mathbf{D}) \mathbf{v}_{\varepsilon}(\mathbf{x}, \tau)+\mathbf{F}(\mathbf{x}, \tau), \\
\mathbf{v}_{\varepsilon}(\mathbf{x}, 0) & =\boldsymbol{\phi}(\mathbf{x}), \quad \frac{\partial \mathbf{v}_{\varepsilon}}{\partial \tau}(\mathbf{x}, 0)=\boldsymbol{\psi}(\mathbf{x}),
\end{aligned}
$$

where $\boldsymbol{\phi}, \boldsymbol{\psi} \in L_{2}\left(\mathbb{R}^{d} ; \mathbb{C}^{n}\right)$ and $\mathbf{F} \in L_{1, \text { loc }}\left(\mathbb{R} ; L_{2}\left(\mathbb{R}^{d} ; \mathbb{C}^{n}\right)\right)$ are given functions. The solution of this problem is represented as

$$
\mathbf{v}_{\varepsilon}(\cdot, \tau)=\cos \left(\tau \widehat{\mathcal{A}}_{\varepsilon}^{1 / 2}\right) \phi+\widehat{\mathcal{A}}_{\varepsilon}^{-1 / 2} \sin \left(\tau \widehat{\mathcal{A}}_{\varepsilon}^{1 / 2}\right) \boldsymbol{\psi}+\int_{0}^{\tau} \widehat{\mathcal{A}}_{\varepsilon}^{-1 / 2} \sin \left((\tau-\widetilde{\tau}) \widehat{\mathcal{A}}_{\varepsilon}^{1 / 2}\right) \mathbf{F}(\cdot, \widetilde{\tau}) d \widetilde{\tau} .
$$

Let $\mathbf{v}_{0}(\mathbf{x}, \tau)$ be the solution of the homogenized problem

$$
\begin{aligned}
\frac{\partial^{2} \mathbf{v}_{0}(\mathbf{x}, \tau)}{\partial \tau^{2}} & =-b(\mathbf{D})^{*} g^{0} b(\mathbf{D}) \mathbf{v}_{0}(\mathbf{x}, \tau)+\mathbf{F}(\mathbf{x}, \tau), \\
\mathbf{v}_{0}(\mathbf{x}, 0) & =\boldsymbol{\phi}(\mathbf{x}), \quad \frac{\partial \mathbf{v}_{0}}{\partial \tau}(\mathbf{x}, 0)=\boldsymbol{\psi}(\mathbf{x}) .
\end{aligned}
$$


Then

$$
\begin{aligned}
\mathbf{v}_{0}(\cdot, \tau)=\cos \left(\tau\left(\widehat{\mathcal{A}}^{0}\right)^{1 / 2}\right) \boldsymbol{\phi} & +\left(\widehat{\mathcal{A}}^{0}\right)^{-1 / 2} \sin \left(\tau\left(\widehat{\mathcal{A}}^{0}\right)^{1 / 2}\right) \boldsymbol{\psi} \\
& +\int_{0}^{\tau}\left(\widehat{\mathcal{A}}^{0}\right)^{-1 / 2} \sin \left((\tau-\widetilde{\tau})\left(\widehat{\mathcal{A}}^{0}\right)^{1 / 2}\right) \mathbf{F}(\cdot, \widetilde{\tau}) d \widetilde{\tau}
\end{aligned}
$$

Now, under the assumptions $\boldsymbol{\phi}, \boldsymbol{\psi} \in H^{s}\left(\mathbb{R}^{d} ; \mathbb{C}^{n}\right)$ and $\mathbf{F} \in L_{1, \text { loc }}\left(\mathbb{R} ; H^{s}\left(\mathbb{R}^{d} ; \mathbb{C}^{n}\right)\right)$ with some $s \in[0,2]$, estimates (13.5) and (13.6) imply that for $\tau \in \mathbb{R}$ and $0<\varepsilon \leq 1$ we have

$$
\begin{aligned}
& \left\|\mathbf{v}_{\varepsilon}(\cdot, \tau)-\mathbf{v}_{0}(\cdot, \tau)\right\|_{L_{2}\left(\mathbb{R}^{d}\right)} \\
& \quad \leq \varepsilon^{s / 2}\left(\widehat{\mathfrak{C}}_{s}(\tau)\|\phi\|_{H^{s}\left(\mathbb{R}^{d}\right)}+\widehat{\mathfrak{C}}_{s}^{\prime}(\tau)\|\boldsymbol{\psi}\|_{H^{s}\left(\mathbb{R}^{d}\right)}\right)+\varepsilon^{s / 2}\left|\int_{0}^{\tau} \widehat{\mathfrak{C}}_{s}^{\prime}(\tau-\widetilde{\tau})\|\mathbf{F}(\cdot, \widetilde{\tau})\|_{H^{s}\left(\mathbb{R}^{d}\right)} d \widetilde{\tau}\right| \\
& \quad \leq \varepsilon^{s / 2}\left(\widehat{\mathfrak{C}}_{s}(\tau)\|\phi\|_{H^{s}\left(\mathbb{R}^{d}\right)}+\widehat{\mathfrak{C}}_{s}^{\prime}(\tau)\|\boldsymbol{\psi}\|_{H^{s}\left(\mathbb{R}^{d}\right)}+\widehat{\mathfrak{C}}_{s}^{\prime}(\tau)\|\mathbf{F}\|_{L_{1}\left((0, \tau) ; H^{s}\left(\mathbb{R}^{d}\right)\right)}\right) .
\end{aligned}
$$

If, moreover, it is known that $\mathbf{F} \in L_{p}\left(\mathbb{R}_{ \pm} ; H^{s}\left(\mathbb{R}^{d} ; \mathbb{C}^{n}\right)\right)$ for some $p \in[1, \infty]$, then, by (13.8) and (13.9), for $\tau= \pm \varepsilon^{-\alpha}, 0<\alpha<s\left(s+2+2 / p^{\prime}\right)^{-1}$, and $0<\varepsilon \leq 1$, we have

$$
\begin{aligned}
& \left\|\mathbf{v}_{\varepsilon}\left(\cdot, \pm \varepsilon^{-\alpha}\right)-\mathbf{v}_{0}\left(\cdot, \pm \varepsilon^{-\alpha}\right)\right\|_{L_{2}\left(\mathbb{R}^{d}\right)} \\
& \leq \varepsilon^{s(1-\alpha) / 2}\left(\widehat{\mathfrak{C}}_{s}(1)\|\boldsymbol{\phi}\|_{H^{s}\left(\mathbb{R}^{d}\right)}+\varepsilon^{-\alpha} \widehat{\mathfrak{C}}_{s}^{\prime}(1)\|\boldsymbol{\psi}\|_{H^{s}\left(\mathbb{R}^{d}\right)}+\varepsilon^{-\alpha} \widehat{\mathfrak{C}}_{s}^{\prime}(1)\left|\int_{0}^{ \pm \varepsilon^{-\alpha}}\|\mathbf{F}(\cdot, \widetilde{\tau})\|_{H^{s}\left(\mathbb{R}^{d}\right)} d \widetilde{\tau}\right|\right) \\
& \leq \varepsilon^{s(1-\alpha) / 2}\left(\widehat{\mathfrak{C}}_{s}(1)\|\boldsymbol{\phi}\|_{H^{s}\left(\mathbb{R}^{d}\right)}+\varepsilon^{-\alpha} \widehat{\mathfrak{C}}_{s}^{\prime}(1)\|\boldsymbol{\psi}\|_{H^{s}\left(\mathbb{R}^{d}\right)}+\varepsilon^{-\alpha-\alpha / p^{\prime}} \widehat{\mathfrak{C}}_{s}^{\prime}(1)\|\mathbf{F}\|_{L_{p}\left(\mathbb{R}_{ \pm} ; H^{s}\left(\mathbb{R}^{d}\right)\right)}\right) .
\end{aligned}
$$

Thus, we have proved the following theorem.

Theorem 15.2. Let $\mathbf{v}_{\varepsilon}$ be the solution of problem (15.5), and $\mathbf{v}_{0}$ the solution of problem (15.6).

$1^{\circ}$. If $\boldsymbol{\phi}, \boldsymbol{\psi} \in H^{s}\left(\mathbb{R}^{d} ; \mathbb{C}^{n}\right)$ and $\mathbf{F} \in L_{1, \text { loc }}\left(\mathbb{R} ; H^{s}\left(\mathbb{R}^{d} ; \mathbb{C}^{n}\right)\right)$ for some $s \in[0,2]$, then for $\tau \in \mathbb{R}$ and $0<\varepsilon \leq 1$ we have

$$
\begin{aligned}
\left\|\mathbf{v}_{\varepsilon}(\cdot, \tau)-\mathbf{v}_{0}(\cdot, \tau)\right\|_{L_{2}\left(\mathbb{R}^{d}\right)} \leq & \varepsilon^{s / 2} \widehat{\mathfrak{C}}_{s}(\tau)\|\phi\|_{H^{s}\left(\mathbb{R}^{d}\right)} \\
& +\varepsilon^{s / 2} \widehat{\mathfrak{C}}_{s}^{\prime}(\tau)\left(\|\boldsymbol{\psi}\|_{H^{s}\left(\mathbb{R}^{d}\right)}+\|\mathbf{F}\|_{L_{1}\left((0, \tau) ; H^{s}\left(\mathbb{R}^{d}\right)\right)}\right) .
\end{aligned}
$$

If, moreover, $\mathbf{F} \in L_{p}\left(\mathbb{R}_{ \pm} ; H^{s}\left(\mathbb{R}^{d} ; \mathbb{C}^{n}\right)\right)$ (where $\left.1 \leq p \leq \infty\right)$, then for $\tau= \pm \varepsilon^{-\alpha}$, $0<\varepsilon \leq 1$, we have

$$
\begin{aligned}
& \left\|\mathbf{v}_{\varepsilon}\left(\cdot, \pm \varepsilon^{-\alpha}\right)-\mathbf{v}_{0}\left(\cdot, \pm \varepsilon^{-\alpha}\right)\right\|_{L_{2}\left(\mathbb{R}^{d}\right)} \\
& \quad \leq \varepsilon^{s(1-\alpha) / 2}\left(\widehat{\mathfrak{C}}_{s}(1)\|\phi\|_{H^{s}\left(\mathbb{R}^{d}\right)}+\varepsilon^{-\alpha} \widehat{\mathfrak{C}}_{s}^{\prime}(1)\|\boldsymbol{\psi}\|_{H^{s}\left(\mathbb{R}^{d}\right)}+\varepsilon^{-\alpha-\alpha / p^{\prime}} \widehat{\mathfrak{C}}_{s}^{\prime}(1)\|\mathbf{F}\|_{L_{p}\left(\mathbb{R}_{ \pm} ; H^{s}\left(\mathbb{R}^{d}\right)\right)}\right) .
\end{aligned}
$$

Here $0<\alpha<s\left(s+2+2 / p^{\prime}\right)^{-1}$. The constants $\widehat{\mathfrak{C}}_{s}(\tau)$ and $\widehat{\mathfrak{C}}_{s}^{\prime}(\tau)$ are defined by (13.7).

$2^{\circ}$. If $\boldsymbol{\phi}, \boldsymbol{\psi} \in L_{2}\left(\mathbb{R}^{d} ; \mathbb{C}^{n}\right)$ and $\mathbf{F} \in L_{1, \mathrm{loc}}\left(\mathbb{R} ; L_{2}\left(\mathbb{R}^{d} ; \mathbb{C}^{n}\right)\right)$, then

$$
\lim _{\varepsilon \rightarrow 0}\left\|\mathbf{v}_{\varepsilon}(\cdot, \tau)-\mathbf{v}_{0}(\cdot, \tau)\right\|_{L_{2}\left(\mathbb{R}^{d}\right)}=0, \quad \tau \in \mathbb{R} .
$$

By the Banach-Steinhaus theorem, statement $2^{\circ}$ follows from (15.7) and the obvious estimate

$$
\left\|\mathbf{v}_{\varepsilon}(\cdot, \tau)-\mathbf{v}_{0}(\cdot, \tau)\right\|_{L_{2}\left(\mathbb{R}^{d}\right)} \leq 2\|\phi\|_{L_{2}\left(\mathbb{R}^{d}\right)}+2|\tau|\|\boldsymbol{\psi}\|_{L_{2}\left(\mathbb{R}^{d}\right)}+2|\tau|\|\mathbf{F}\|_{L_{1}\left((0, \tau) ; L_{2}\left(\mathbb{R}^{d}\right)\right)} .
$$

15.3. The Cauchy problem for equation (11.4) with the operator $\mathcal{A}_{\varepsilon}$. Let $\mathcal{A}_{\varepsilon}$ be the operator (11.2). We consider the following Cauchy problem for a function $\mathbf{v}_{\varepsilon}(\mathbf{x}, \tau)$, $\mathbf{x} \in \mathbb{R}^{d}, \tau \in \mathbb{R}:$

$$
\begin{aligned}
\frac{\partial^{2} \mathbf{v}_{\varepsilon}(\mathbf{x}, \tau)}{\partial \tau^{2}} & =-\left(f^{\varepsilon}(\mathbf{x})\right)^{*} b(\mathbf{D})^{*} g^{\varepsilon}(\mathbf{x}) b(\mathbf{D}) f^{\varepsilon}(\mathbf{x}) \mathbf{v}_{\varepsilon}(\mathbf{x}, \tau), \\
f^{\varepsilon}(\mathbf{x}) \mathbf{v}_{\varepsilon}(\mathbf{x}, 0) & =\phi(\mathbf{x}), \quad f^{\varepsilon}(\mathbf{x}) \frac{\partial \mathbf{v}_{\varepsilon}}{\partial \tau}(\mathbf{x}, 0)=\boldsymbol{\psi}(\mathbf{x}),
\end{aligned}
$$


where $\phi, \boldsymbol{\psi} \in L_{2}\left(\mathbb{R}^{d} ; \mathbb{C}^{n}\right)$ are given functions. The solution $\mathbf{v}_{\varepsilon}$ of (15.8) admits the representation

$$
\mathbf{v}_{\varepsilon}=\cos \left(\tau \mathcal{A}_{\varepsilon}^{1 / 2}\right)\left(f^{\varepsilon}\right)^{-1} \boldsymbol{\phi}+\mathcal{A}_{\varepsilon}^{-1 / 2} \sin \left(\tau \mathcal{A}_{\varepsilon}^{1 / 2}\right)\left(f^{\varepsilon}\right)^{-1} \boldsymbol{\psi}
$$

Let $\mathbf{v}_{0}(\mathbf{x}, \tau)$ be the solution of the "homogenized" Cauchy problem

$$
\begin{aligned}
\frac{\partial^{2} \mathbf{v}_{0}(\mathbf{x}, \tau)}{\partial \tau^{2}} & =-f_{0} b(\mathbf{D})^{*} g^{0} b(\mathbf{D}) f_{0} \mathbf{v}_{0}(\mathbf{x}, \tau) \\
f_{0} \mathbf{v}_{0}(\mathbf{x}, 0) & =\boldsymbol{\phi}(\mathbf{x}), \quad f_{0} \frac{\partial \mathbf{v}_{0}}{\partial \tau}(\mathbf{x}, 0)=\boldsymbol{\psi}(\mathbf{x}) .
\end{aligned}
$$

Here $g^{0}$ is the effective matrix, and $f_{0}$ is the matrix defined by (8.2). Then $\mathbf{v}_{0}=$ $\cos \left(\tau\left(\mathcal{A}^{0}\right)^{1 / 2}\right) f_{0}^{-1} \boldsymbol{\phi}+\left(\mathcal{A}^{0}\right)^{-1 / 2} \sin \left(\tau\left(\mathcal{A}^{0}\right)^{1 / 2}\right) f_{0}^{-1} \boldsymbol{\psi}$.

Theorem 13.4 implies the following result.

Theorem 15.3. Let $\mathbf{v}_{\varepsilon}$ be the solution of problem (15.8), and $\mathbf{v}_{0}$ the solution of problem (15.9).

$1^{\circ}$. If $\boldsymbol{\phi}, \boldsymbol{\psi} \in H^{s}\left(\mathbb{R}^{d} ; \mathbb{C}^{n}\right), 0 \leq s \leq 2$, then for $\tau \in \mathbb{R}$ and $0<\varepsilon \leq 1$ we have

$$
\left\|f^{\varepsilon} \mathbf{v}_{\varepsilon}(\cdot, \tau)-f_{0} \mathbf{v}_{0}(\cdot, \tau)\right\|_{L_{2}\left(\mathbb{R}^{d}\right)} \leq \varepsilon^{s / 2}\left(\mathfrak{C}_{s}(\tau)\|\phi\|_{H^{s}\left(\mathbb{R}^{d}\right)}+\mathfrak{C}_{s}^{\prime}(\tau)\|\boldsymbol{\psi}\|_{H^{s}\left(\mathbb{R}^{d}\right)}\right) .
$$

In particular, for $\tau= \pm \varepsilon^{-\alpha}$ and $0<\varepsilon \leq 1$ we have

$$
\begin{aligned}
\left\|f^{\varepsilon} \mathbf{v}_{\varepsilon}\left(\cdot, \pm \varepsilon^{-\alpha}\right)-f_{0} \mathbf{v}_{0}\left(\cdot, \pm \varepsilon^{-\alpha}\right)\right\|_{L_{2}\left(\mathbb{R}^{d}\right)} & \\
& \leq \varepsilon^{s(1-\alpha) / 2}\left(\mathfrak{C}_{s}(1)\|\phi\|_{H^{s}\left(\mathbb{R}^{d}\right)}+\varepsilon^{-\alpha} \mathfrak{C}_{s}^{\prime}(1)\|\boldsymbol{\psi}\|_{H^{s}\left(\mathbb{R}^{d}\right)}\right) .
\end{aligned}
$$

Here $0<\alpha<s(s+2)^{-1}$ if $\boldsymbol{\psi} \neq 0$, and $0<\alpha<1$ if $\boldsymbol{\psi}=0$. The constants $\mathfrak{C}_{s}(\tau)$ and $\mathfrak{C}_{s}^{\prime}(\tau)$ are defined by $(13.12)$.

$2^{\circ}$. If $\boldsymbol{\phi}, \boldsymbol{\psi} \in L_{2}\left(\mathbb{R}^{d} ; \mathbb{C}^{n}\right)$, then

$$
\lim _{\varepsilon \rightarrow 0}\left\|f^{\varepsilon} \mathbf{v}_{\varepsilon}(\cdot, \tau)-f_{0} \mathbf{v}_{0}(\cdot, \tau)\right\|_{L_{2}\left(\mathbb{R}^{d}\right)}=0, \quad \tau \in \mathbb{R} .
$$

$3^{\circ}$. If $\phi \in L_{2}\left(\mathbb{R}^{d} ; \mathbb{C}^{n}\right)$ and $\boldsymbol{\psi}=0$, then

$$
\lim _{\varepsilon \rightarrow 0}\left\|f^{\varepsilon} \mathbf{v}_{\varepsilon}\left(\cdot, \pm \varepsilon^{-\alpha}\right)-f_{0} \mathbf{v}_{0}\left(\cdot, \pm \varepsilon^{-\alpha}\right)\right\|_{L_{2}\left(\mathbb{R}^{d}\right)}=0, \quad 0<\alpha<1 .
$$

Note that, by the substitution $f^{\varepsilon} \mathbf{v}_{\varepsilon}=\mathbf{z}_{\varepsilon}$, problem (15.8) admits the following reformulation:

$$
\begin{aligned}
Q^{\varepsilon}(\mathbf{x}) \frac{\partial^{2} \mathbf{z}_{\varepsilon}(\mathbf{x}, \tau)}{\partial \tau^{2}} & =-b(\mathbf{D})^{*} g^{\varepsilon}(\mathbf{x}) b(\mathbf{D}) \mathbf{z}_{\varepsilon}(\mathbf{x}, \tau), \\
\mathbf{z}_{\varepsilon}(\mathbf{x}, 0) & =\boldsymbol{\phi}(\mathbf{x}), \quad \frac{\partial \mathbf{z}_{\varepsilon}}{\partial \tau}(\mathbf{x}, 0)=\boldsymbol{\psi}(\mathbf{x}),
\end{aligned}
$$

where $Q^{\varepsilon}(\mathbf{x})=\left(f^{\varepsilon}(\mathbf{x})\left(f^{\varepsilon}(\mathbf{x})\right)^{*}\right)^{-1}$. By the substitution $f_{0} \mathbf{v}_{0}=\mathbf{z}_{0}$, the homogenized problem (15.9) turns into the problem

$$
\begin{aligned}
\bar{Q} \frac{\partial^{2} \mathbf{z}_{0}(\mathbf{x}, \tau)}{\partial \tau^{2}} & =-b(\mathbf{D})^{*} g^{0} b(\mathbf{D}) \mathbf{z}_{0}(\mathbf{x}, \tau) \\
\mathbf{z}_{0}(\mathbf{x}, 0) & =\boldsymbol{\phi}(\mathbf{x}), \quad \frac{\partial \mathbf{z}_{0}}{\partial \tau}(\mathbf{x}, 0)=\boldsymbol{\psi}(\mathbf{x}),
\end{aligned}
$$

where $\bar{Q}$ is the mean value of the matrix $Q(\mathbf{x})$ over $\Omega$.

If (15.10) is regarded as the initial statement of the problem, then it is assumed that $Q(\mathbf{x})$ is a $\Gamma$-periodic matrix-valued function satisfying condition (14.14).

Theorem 15.3 admits the following equivalent formulation. 
Theorem 15.4. Let $\mathbf{z}_{\varepsilon}$ be the solution of problem (15.10), and $\mathbf{z}_{0}$ the solution of problem (15.11).

$1^{\circ}$. If $\boldsymbol{\phi}, \boldsymbol{\psi} \in H^{s}\left(\mathbb{R}^{d} ; \mathbb{C}^{n}\right), 0 \leq s \leq 2$, then for $\tau \in \mathbb{R}$ and $0<\varepsilon \leq 1$ we have

$$
\left\|\mathbf{z}_{\varepsilon}(\cdot, \tau)-\mathbf{z}_{0}(\cdot, \tau)\right\|_{L_{2}\left(\mathbb{R}^{d}\right)} \leq \varepsilon^{s / 2}\left(\mathfrak{C}_{s}(\tau)\|\phi\|_{H^{s}\left(\mathbb{R}^{d}\right)}+\mathfrak{C}_{s}^{\prime}(\tau)\|\boldsymbol{\psi}\|_{H^{s}\left(\mathbb{R}^{d}\right)}\right) .
$$

In particular, for $\tau= \pm \varepsilon^{-\alpha}$ and $0<\varepsilon \leq 1$ we have

$$
\left\|\mathbf{z}_{\varepsilon}\left(\cdot, \pm \varepsilon^{-\alpha}\right)-\mathbf{z}_{0}\left(\cdot, \pm \varepsilon^{-\alpha}\right)\right\|_{L_{2}\left(\mathbb{R}^{d}\right)} \leq \varepsilon^{s(1-\alpha) / 2}\left(\mathfrak{C}_{s}(1)\|\phi\|_{H^{s}\left(\mathbb{R}^{d}\right)}+\varepsilon^{-\alpha} \mathfrak{C}_{s}^{\prime}(1)\|\boldsymbol{\psi}\|_{H^{s}\left(\mathbb{R}^{d}\right)}\right) .
$$

Here $0<\alpha<s(s+2)^{-1}$ if $\boldsymbol{\psi} \neq 0$, and $0<\alpha<1$ if $\boldsymbol{\psi}=0$. The constants $\mathfrak{C}_{s}(\tau)$ and $\mathfrak{C}_{s}^{\prime}(\tau)$ are defined by $(13.12)$.

$2^{\circ}$. If $\boldsymbol{\phi}, \boldsymbol{\psi} \in L_{2}\left(\mathbb{R}^{d} ; \mathbb{C}^{n}\right)$, then

$$
\lim _{\varepsilon \rightarrow 0}\left\|\mathbf{z}_{\varepsilon}(\cdot, \tau)-\mathbf{z}_{0}(\cdot, \tau)\right\|_{L_{2}\left(\mathbb{R}^{d}\right)}=0, \quad \tau \in \mathbb{R} .
$$

$3^{\circ}$. If $\phi \in L_{2}\left(\mathbb{R}^{d} ; \mathbb{C}^{n}\right)$ and $\boldsymbol{\psi}=0$, then

$$
\lim _{\varepsilon \rightarrow 0}\left\|\mathbf{z}_{\varepsilon}\left(\cdot, \pm \varepsilon^{-\alpha}\right)-\mathbf{z}_{0}\left(\cdot, \pm \varepsilon^{-\alpha}\right)\right\|_{L_{2}\left(\mathbb{R}^{d}\right)}=0, \quad 0<\alpha<1 .
$$

15.4. The nonhomogeneous Cauchy problem with the operator $\mathcal{A}_{\varepsilon}$. Now we consider a problem more general than (15.8):

$$
\begin{aligned}
\frac{\partial^{2} \mathbf{v}_{\varepsilon}(\mathbf{x}, \tau)}{\partial \tau^{2}} & =-\left(f^{\varepsilon}(\mathbf{x})\right)^{*} b(\mathbf{D})^{*} g^{\varepsilon}(\mathbf{x}) b(\mathbf{D}) f^{\varepsilon}(\mathbf{x}) \mathbf{v}_{\varepsilon}(\mathbf{x}, \tau)+\left(f^{\varepsilon}(\mathbf{x})\right)^{-1} \mathbf{F}(\mathbf{x}, \tau), \\
f^{\varepsilon}(\mathbf{x}) \mathbf{v}_{\varepsilon}(\mathbf{x}, 0) & =\phi(\mathbf{x}), \quad f^{\varepsilon}(\mathbf{x}) \frac{\partial \mathbf{v}_{\varepsilon}}{\partial \tau}(\mathbf{x}, 0)=\boldsymbol{\psi}(\mathbf{x}),
\end{aligned}
$$

where $\boldsymbol{\phi}, \boldsymbol{\psi} \in L_{2}\left(\mathbb{R}^{d} ; \mathbb{C}^{n}\right)$ and $\mathbf{F} \in L_{1, \text { loc }}\left(\mathbb{R} ; L_{2}\left(\mathbb{R}^{d} ; \mathbb{C}^{n}\right)\right)$ are given functions. The solution of this problem is represented as

$$
\begin{aligned}
\mathbf{v}_{\varepsilon}(\cdot, \tau)=\cos \left(\tau \mathcal{A}_{\varepsilon}^{1 / 2}\right)\left(f^{\varepsilon}\right)^{-1} \phi & +\mathcal{A}_{\varepsilon}^{-1 / 2} \sin \left(\tau \mathcal{A}_{\varepsilon}^{1 / 2}\right)\left(f^{\varepsilon}\right)^{-1} \boldsymbol{\psi} \\
& +\int_{0}^{\tau} \mathcal{A}_{\varepsilon}^{-1 / 2} \sin \left((\tau-\widetilde{\tau}) \mathcal{A}_{\varepsilon}^{1 / 2}\right)\left(f^{\varepsilon}\right)^{-1} \mathbf{F}(\cdot, \widetilde{\tau}) d \widetilde{\tau} .
\end{aligned}
$$

Let $\mathbf{v}_{0}(\mathbf{x}, \tau)$ be the solution of the homogenized problem

$$
\begin{aligned}
\frac{\partial^{2} \mathbf{v}_{0}(\mathbf{x}, \tau)}{\partial \tau^{2}} & =-f_{0} b(\mathbf{D})^{*} g^{0} b(\mathbf{D}) f_{0} \mathbf{v}_{0}(\mathbf{x}, \tau)+f_{0}^{-1} \mathbf{F}(\mathbf{x}, \tau), \\
f_{0} \mathbf{v}_{0}(\mathbf{x}, 0) & =\boldsymbol{\phi}(\mathbf{x}), \quad f_{0} \frac{\partial \mathbf{v}_{0}}{\partial \tau}(\mathbf{x}, 0)=\boldsymbol{\psi}(\mathbf{x}) .
\end{aligned}
$$

Then

$$
\begin{aligned}
\mathbf{v}_{0}(\cdot, \tau)=\cos \left(\tau\left(\mathcal{A}^{0}\right)^{1 / 2}\right) f_{0}^{-1} \phi & +\left(\mathcal{A}^{0}\right)^{-1 / 2} \sin \left(\tau\left(\mathcal{A}^{0}\right)^{1 / 2}\right) f_{0}^{-1} \boldsymbol{\psi} \\
& +\int_{0}^{\tau}\left(\mathcal{A}^{0}\right)^{-1 / 2} \sin \left((\tau-\widetilde{\tau})\left(\mathcal{A}^{0}\right)^{1 / 2}\right) f_{0}^{-1} \mathbf{F}(\cdot, \widetilde{\tau}) d \widetilde{\tau} .
\end{aligned}
$$

In the same way as Theorem 15.2 was deduced from Theorem 13.2, an application of Theorem 13.4 implies the following result.

Theorem 15.5. Let $\mathbf{v}_{\varepsilon}$ be the solution of problem (15.12), and $\mathbf{v}_{0}$ the solution of problem (15.13).

$1^{\circ}$. If $\boldsymbol{\phi}, \boldsymbol{\psi} \in H^{s}\left(\mathbb{R}^{d} ; \mathbb{C}^{n}\right)$ and $\mathbf{F} \in L_{1, \text { loc }}\left(\mathbb{R} ; H^{s}\left(\mathbb{R}^{d} ; \mathbb{C}^{n}\right)\right)$ for some $s \in[0,2]$, then for $\tau \in \mathbb{R}$ and $0<\varepsilon \leq 1$ we have

$$
\begin{aligned}
\left\|f^{\varepsilon} \mathbf{v}_{\varepsilon}(\cdot, \tau)-f_{0} \mathbf{v}_{0}(\cdot, \tau)\right\|_{L_{2}\left(\mathbb{R}^{d}\right)} \leq & \varepsilon^{s / 2} \mathfrak{C}_{s}(\tau)\|\phi\|_{H^{s}\left(\mathbb{R}^{d}\right)} \\
& +\varepsilon^{s / 2} \mathfrak{C}_{s}^{\prime}(\tau)\left(\|\boldsymbol{\psi}\|_{H^{s}\left(\mathbb{R}^{d}\right)}+\|\mathbf{F}\|_{L_{1}\left((0, \tau) ; H^{s}\left(\mathbb{R}^{d}\right)\right)}\right) .
\end{aligned}
$$


If, moreover, $\mathbf{F} \in L_{p}\left(\mathbb{R}_{ \pm} ; H^{s}\left(\mathbb{R}^{d} ; \mathbb{C}^{n}\right)\right.$ ) (where $\left.1 \leq p \leq \infty\right)$, then for $\tau= \pm \varepsilon^{-\alpha}$, $0<\varepsilon \leq 1$, we have

$$
\begin{aligned}
& \left\|f^{\varepsilon} \mathbf{v}_{\varepsilon}\left(\cdot, \pm \varepsilon^{-\alpha}\right)-f_{0} \mathbf{v}_{0}\left(\cdot, \pm \varepsilon^{-\alpha}\right)\right\|_{L_{2}\left(\mathbb{R}^{d}\right)} \\
& \quad \leq \varepsilon^{s(1-\alpha) / 2}\left(\mathfrak{C}_{s}(1)\|\phi\|_{H^{s}\left(\mathbb{R}^{d}\right)}+\varepsilon^{-\alpha} \mathfrak{C}_{s}^{\prime}(1)\|\boldsymbol{\psi}\|_{H^{s}\left(\mathbb{R}^{d}\right)}+\varepsilon^{-\alpha-\alpha / p^{\prime}} \mathfrak{C}_{s}^{\prime}(1)\|\mathbf{F}\|_{L_{p}\left(\mathbb{R}_{ \pm} ; H^{s}\left(\mathbb{R}^{d}\right)\right)}\right) .
\end{aligned}
$$

Here $0<\alpha<s\left(s+2+2 / p^{\prime}\right)^{-1}$. The constants $\mathfrak{C}_{s}(\tau)$ and $\mathfrak{C}_{s}^{\prime}(\tau)$ are defined by (13.12).

$2^{\circ}$. If $\boldsymbol{\phi}, \boldsymbol{\psi} \in L_{2}\left(\mathbb{R}^{d} ; \mathbb{C}^{n}\right)$ and $\mathbf{F} \in L_{1, \text { loc }}\left(\mathbb{R} ; L_{2}\left(\mathbb{R}^{d} ; \mathbb{C}^{n}\right)\right)$, then

$$
\lim _{\varepsilon \rightarrow 0}\left\|f^{\varepsilon} \mathbf{v}_{\varepsilon}(\cdot, \tau)-f_{0} \mathbf{v}_{0}(\cdot, \tau)\right\|_{L_{2}\left(\mathbb{R}^{d}\right)}=0, \quad \tau \in \mathbb{R} .
$$

Finally, we note that, by the substitution $f^{\varepsilon} \mathbf{v}_{\varepsilon}=\mathbf{z}_{\varepsilon}$, problem (15.12) turns into the following problem:

$$
\begin{aligned}
Q^{\varepsilon}(\mathbf{x}) \frac{\partial^{2} \mathbf{z}_{\varepsilon}(\mathbf{x}, \tau)}{\partial \tau^{2}} & =-b(\mathbf{D})^{*} g^{\varepsilon}(\mathbf{x}) b(\mathbf{D}) \mathbf{z}_{\varepsilon}(\mathbf{x}, \tau)+Q^{\varepsilon}(\mathbf{x}) \mathbf{F}(\mathbf{x}, \tau), \\
\mathbf{z}_{\varepsilon}(\mathbf{x}, 0) & =\boldsymbol{\phi}(\mathbf{x}), \quad \frac{\partial \mathbf{z}_{\varepsilon}}{\partial \tau}(\mathbf{x}, 0)=\boldsymbol{\psi}(\mathbf{x}),
\end{aligned}
$$

where $Q^{\varepsilon}(\mathbf{x})=\left(f^{\varepsilon}(\mathbf{x})\left(f^{\varepsilon}(\mathbf{x})\right)^{*}\right)^{-1}$. By the substitution $f_{0} \mathbf{v}_{0}=\mathbf{z}_{0}$, the homogenized problem (15.13) turns into

$$
\begin{aligned}
\bar{Q} \frac{\partial^{2} \mathbf{z}_{0}(\mathbf{x}, \tau)}{\partial \tau^{2}} & =-b(\mathbf{D})^{*} g^{0} b(\mathbf{D}) \mathbf{z}_{0}(\mathbf{x}, \tau)+\bar{Q} \mathbf{F}(\mathbf{x}, \tau), \\
\mathbf{z}_{0}(\mathbf{x}, 0) & =\boldsymbol{\phi}(\mathbf{x}), \quad \frac{\partial \mathbf{z}_{0}}{\partial \tau}(\mathbf{x}, 0)=\boldsymbol{\psi}(\mathbf{x}) .
\end{aligned}
$$

If (15.14) is regarded as the initial statement of the problem, then it is assumed that $Q(\mathbf{x})$ is a $\Gamma$-periodic matrix-valued function satisfying (14.14).

Theorem 15.5 can be reformulated in the following way.

Theorem 15.6. Let $\mathbf{z}_{\varepsilon}$ be the solution of problem (15.14), and $\mathbf{z}_{0}$ the solution of problem (15.15).

$1^{\circ}$. If $\boldsymbol{\phi}, \boldsymbol{\psi} \in H^{s}\left(\mathbb{R}^{d} ; \mathbb{C}^{n}\right)$ and $\mathbf{F} \in L_{1, \text { loc }}\left(\mathbb{R} ; H^{s}\left(\mathbb{R}^{d} ; \mathbb{C}^{n}\right)\right)$ for some $s \in[0,2]$, then for $\tau \in \mathbb{R}$ and $0<\varepsilon \leq 1$ we have

$$
\begin{aligned}
\left\|\mathbf{z}_{\varepsilon}(\cdot, \tau)-\mathbf{z}_{0}(\cdot, \tau)\right\|_{L_{2}\left(\mathbb{R}^{d}\right)} \leq & \varepsilon^{s / 2} \mathfrak{C}_{s}(\tau)\|\phi\|_{H^{s}\left(\mathbb{R}^{d}\right)} \\
& +\varepsilon^{s / 2} \mathfrak{C}_{s}^{\prime}(\tau)\left(\|\boldsymbol{\psi}\|_{H^{s}\left(\mathbb{R}^{d}\right)}+\|\mathbf{F}\|_{L_{1}\left((0, \tau) ; H^{s}\left(\mathbb{R}^{d}\right)\right)}\right) .
\end{aligned}
$$

If, moreover, $\mathbf{F} \in L_{p}\left(\mathbb{R}_{ \pm} ; H^{s}\left(\mathbb{R}^{d} ; \mathbb{C}^{n}\right)\right.$ ) (where $\left.1 \leq p \leq \infty\right)$, then for $\tau= \pm \varepsilon^{-\alpha}$, $0<\varepsilon \leq 1$, we have

$$
\begin{aligned}
& \left\|\mathbf{z}_{\varepsilon}\left(\cdot, \pm \varepsilon^{-\alpha}\right)-\mathbf{z}_{0}\left(\cdot, \pm \varepsilon^{-\alpha}\right)\right\|_{L_{2}\left(\mathbb{R}^{d}\right)} \\
& \quad \leq \varepsilon^{s(1-\alpha) / 2}\left(\mathfrak{C}_{s}(1)\|\phi\|_{H^{s}\left(\mathbb{R}^{d}\right)}+\varepsilon^{-\alpha} \mathfrak{C}_{s}^{\prime}(1)\|\boldsymbol{\psi}\|_{H^{s}\left(\mathbb{R}^{d}\right)}+\varepsilon^{-\alpha-\alpha / p^{\prime}} \mathfrak{C}_{s}^{\prime}(1)\|\mathbf{F}\|_{L_{p}\left(\mathbb{R}_{ \pm} ; H^{s}\left(\mathbb{R}^{d}\right)\right)}\right) .
\end{aligned}
$$

Here $0<\alpha<s\left(s+2+2 / p^{\prime}\right)^{-1}$. The constants $\mathfrak{C}_{s}(\tau)$ and $\mathfrak{C}_{s}^{\prime}(\tau)$ are defined by (13.12).

$2^{\circ}$. If $\boldsymbol{\phi}, \boldsymbol{\psi} \in L_{2}\left(\mathbb{R}^{d} ; \mathbb{C}^{n}\right)$ and $\mathbf{F} \in L_{1, \text { loc }}\left(\mathbb{R} ; L_{2}\left(\mathbb{R}^{d} ; \mathbb{C}^{n}\right)\right)$, then

$$
\lim _{\varepsilon \rightarrow 0}\left\|\mathbf{z}_{\varepsilon}(\cdot, \tau)-\mathbf{z}_{0}(\cdot, \tau)\right\|_{L_{2}\left(\mathbb{R}^{d}\right)}=0, \quad \tau \in \mathbb{R} .
$$

\section{Chapter 5. Applications of general Results}

We proceed to applications of our general results to specific equations of mathematical physics. All operators of the form (11.1), (11.2) that are considered in the present chapter have been studied before; see [BSu2, Chapter 5, 6], BSu4, Chapter 4], BSu5, Chapter 4]. The necessary facts about factorization and the effective characteristics for these operators are borrowed from $\mathrm{BSu2}$, Chapters 5, 6]. 
In $\S 16$, we consider the model example of the Schrödinger-type equation with the operator $\widehat{\mathcal{A}}_{\varepsilon}=-\operatorname{div} g^{\varepsilon} \nabla$, and the Schrödinger equation itself with the Hamiltonian $\mathcal{H}_{\varepsilon}=$ $-\operatorname{div} \widetilde{g}^{\varepsilon} \nabla+\varepsilon^{-2} V^{\varepsilon}$. $\S 17$ is devoted to the two-dimensional Pauli equation; beforehand, we consider some model examples. In $\S 18$, we study hyperbolic equations, namely, the nonstationary acoustics equation and the system of elasticity theory.

\section{§16. The Schrödinger Equation}

16.1. The model example. The Schrödinger-type equation with the operator $\widehat{\mathcal{A}}_{\varepsilon}=-\operatorname{div} g^{\varepsilon}(\mathbf{x}) \nabla$. In $L_{2}\left(\mathbb{R}^{d}\right), d \geq 1$, we consider the operator

$$
\widehat{\mathcal{A}}=\mathbf{D}^{*} g(\mathbf{x}) \mathbf{D}=-\operatorname{div} g(\mathbf{x}) \nabla .
$$

Here $g(\mathbf{x})$ is a $\Gamma$-periodic $(d \times d)$-matrix-valued function with real entries such that

$$
g(\mathbf{x})>0 ; \quad g, g^{-1} \in L_{\infty} .
$$

The operator (16.1) is a particular case of the operator (6.1). Here, $n=1, m=d$, and $b(\mathbf{D})=\mathbf{D}$. Obviously, condition (4.2) is satisfied with $\alpha_{0}=\alpha_{1}=1$. To realize the representation $g(\mathbf{x})=h(\mathbf{x})^{*} h(\mathbf{x})$ we can, for instance, put $h=g^{1 / 2}$.

In accordance with (6.8), the effective operator for (16.1) has the form

$$
\widehat{\mathcal{A}}^{0}=\mathbf{D}^{*} g^{0} \mathbf{D}=-\operatorname{div} g^{0} \nabla .
$$

By the general rules, the effective matrix $g^{0}$ is defined in the following way. Let $\mathbf{e}_{1}, \ldots, \mathbf{e}_{d}$ be the standard orthonormal basis in $\mathbb{R}^{d}$. Let $\Phi_{j} \in \widetilde{H}^{1}(\Omega)$ be a (weak) periodic solution of the problem

$$
\operatorname{div} g(\mathbf{x})\left(\nabla \Phi_{j}(\mathbf{x})+\mathbf{e}_{j}\right)=0, \quad \int_{\Omega} \Phi_{j}(\mathbf{x}) d \mathbf{x}=0 .
$$

Then $g^{0}$ is the $(d \times d)$-matrix with the columns

$$
g_{j}^{0}=|\Omega|^{-1} \int_{\Omega} g(\mathbf{x})\left(\nabla \Phi_{j}(\mathbf{x})+\mathbf{e}_{j}\right) d \mathbf{x}, \quad j=1, \ldots, d .
$$

In the one-dimensional case, we have $d=m=n=1$, whence $g^{0}=\underline{g}$.

Now we consider the operator

$$
\widehat{\mathcal{A}_{\varepsilon}}=\mathbf{D}^{*} g^{\varepsilon}(\mathbf{x}) \mathbf{D}, \quad \varepsilon>0,
$$

and the following Cauchy problem of the form (14.1):

$$
i \frac{\partial u_{\varepsilon}(\mathbf{x}, \tau)}{\partial \tau}=\mathbf{D}^{*} g^{\varepsilon}(\mathbf{x}) \mathbf{D} u_{\varepsilon}(\mathbf{x}, \tau), \quad u_{\varepsilon}(\mathbf{x}, 0)=\phi(\mathbf{x}),
$$

where $\phi \in L_{2}\left(\mathbb{R}^{d}\right)$ is a given function. The differential equation in (16.7) can be interpreted as the Schrödinger equation with periodic metric in the absence of external fields.

Let $u_{0}(\mathbf{x}, \tau)$ be the solution of the "homogenized" Cauchy problem

$$
i \frac{\partial u_{0}(\mathbf{x}, \tau)}{\partial \tau}=\mathbf{D}^{*} g^{0} \mathbf{D} u_{0}(\mathbf{x}, \tau), \quad u_{0}(\mathbf{x}, 0)=\phi(\mathbf{x}) .
$$

Theorem 14.1 is applicable. If $\phi \in H^{s}\left(\mathbb{R}^{d}\right), 0<s \leq 3$, then, as $\varepsilon \rightarrow 0$, the solution $u_{\varepsilon}$ of problem (16.7) tends to the solution $u_{0}$ of problem (16.8) in the $L_{2}\left(\mathbb{R}^{d}\right)$-norm for any fixed $\tau \in \mathbb{R}$. Herewith,

$$
\left\|u_{\varepsilon}(\cdot, \tau)-u_{0}(\cdot, \tau)\right\|_{L_{2}\left(\mathbb{R}^{d}\right)} \leq \varepsilon^{s / 3} \widehat{\mathcal{C}}_{s}(\tau)\|\phi\|_{H^{s}\left(\mathbb{R}^{d}\right)} .
$$


The constant $\widehat{\mathcal{C}}_{s}(\tau)$ is of order $O\left(|\tau|^{s / 3}\right.$ ) (for large values of $|\tau|$ ) and depends on the norms $\|g\|_{L_{\infty}},\left\|g^{-1}\right\|_{L_{\infty}}$ and on the parameters of the lattice $\Gamma$. For large values of time $|\tau|=\varepsilon^{-\alpha}$ we have

$$
\left\|u_{\varepsilon}\left(\cdot, \pm \varepsilon^{-\alpha}\right)-u_{0}\left(\cdot, \pm \varepsilon^{-\alpha}\right)\right\|_{L_{2}\left(\mathbb{R}^{d}\right)} \leq \varepsilon^{s(1-\alpha) / 3} \widehat{\mathcal{C}}_{s}(1)\|\phi\|_{H^{s}\left(\mathbb{R}^{d}\right)}, \quad 0<\alpha<1 .
$$

If it is only known that $\phi \in L_{2}\left(\mathbb{R}^{d}\right)$, then, as $\varepsilon \rightarrow 0$, the solution $u_{\varepsilon}$ tends to $u_{0}$ strongly in $L_{2}\left(\mathbb{R}^{d}\right)$ for any fixed $\tau \in \mathbb{R}$, and also the difference $u_{\varepsilon}\left(\cdot, \pm \varepsilon^{-\alpha}\right)-u_{0}\left(\cdot, \pm \varepsilon^{-\alpha}\right)$ tends to zero strongly in $L_{2}\left(\mathbb{R}^{d}\right)$, provided $0<\alpha<1$.

Also, the nonhomogeneous problem of the form (14.4) with the operator (16.6) can be studied. Theorem 14.2 may be applied to this problem. We shall not dwell on this.

16.2. The periodic Schrödinger operator. Factorization. (See BSu2, Chapter 6, Subsection 1.1].) In the space $L_{2}\left(\mathbb{R}^{d}\right), d \geq 1$, we consider the periodic Schrödinger operator (with metric) of the form

$$
\mathcal{H}=\mathbf{D}^{*} \widetilde{g}(\mathbf{x}) \mathbf{D}+V(\mathbf{x}),
$$

where a $(d \times d)$-matrix-valued function $\widetilde{g}(\mathbf{x})$ with real entries and a real-valued potential $V(\mathbf{x})$ are $\Gamma$-periodic and satisfy

$$
\begin{gathered}
\tilde{g}(\mathbf{x})>0, \quad \tilde{g}, \widetilde{g}^{-1} \in L_{\infty}, \\
V \in L_{q}(\Omega), \quad 2 q>d \quad \text { for } d \geq 2, \quad q=1 \quad \text { for } d=1 .
\end{gathered}
$$

The precise definition of the selfadjoint operator $\mathcal{H}$ in $L_{2}\left(\mathbb{R}^{d}\right)$ is given in terms of the quadratic form

$$
h[u, u]=\int_{\mathbb{R}^{d}}\left(\langle\widetilde{g}(\mathbf{x}) \mathbf{D} u, \mathbf{D} u\rangle_{\mathbb{C}^{d}}+V(\mathbf{x})|u|^{2}\right) d \mathbf{x}, \quad u \in H^{1}\left(\mathbb{R}^{d}\right) .
$$

Under conditions (16.10) and (16.11), this form is lower semibounded and closed in $L_{2}\left(\mathbb{R}^{d}\right)$. Adding an appropriate constant to $V(\mathbf{x})$, we may assume that the point $\lambda_{0}=0$ is the bottom of the spectrum of $\mathcal{H}$. We assume that the latter condition is satisfied.

Under the above assumptions, the equation $\left(\mathbf{D}^{*} \widetilde{g} \mathbf{D}+V\right) \omega=0$ has a (weak) positive $\Gamma$-periodic solution $\omega \in \widetilde{H}^{1}(\Omega)$, and $\omega \in \operatorname{Lip} \sigma$ (with some $\sigma>0$ ). Moreover, the function $\omega$ is a multiplier in the classes $H^{1}\left(\mathbb{R}^{d}\right)$ and $\widetilde{H}^{1}(\Omega)$. We fix the choice of $\omega$ by the normalization condition

$$
\int_{\Omega} \omega(\mathbf{x})^{2} d \mathbf{x}=|\Omega|
$$

After the substitution $u=\omega v$, the form (16.12) turns into

$$
h[u, u]=\int_{\mathbb{R}^{d}} \omega^{2}\langle\widetilde{g} \mathbf{D} v, \mathbf{D} v\rangle_{\mathbb{C}^{d}} d \mathbf{x}, \quad u=\omega v, \quad v \in H^{1}\left(\mathbb{R}^{d}\right) .
$$

This means that the operator (16.9) is represented as a product:

$$
\mathcal{H}=\omega^{-1} \mathbf{D}^{*} \omega^{2} \widetilde{g} \mathbf{D} \omega^{-1} \text {. }
$$

Thus, the operator $\mathcal{H}$ takes the form (4.6) with $n=1, m=d, b(\mathbf{D})=\mathbf{D}, g=\omega^{2} \widetilde{g}$, and $f=\omega^{-1}$.

Remark 16.1. The expression (16.14) can be taken as the definition of the operator $\mathcal{H}$ for any $\Gamma$-periodic function $\omega$ satisfying condition (16.13) and such that

$$
\omega(\mathbf{x})>0, \quad \omega, \omega^{-1} \in L_{\infty} .
$$

The form (16.9) can be recovered by the formula $V=-\omega^{-1}\left(\mathbf{D}^{*} \widetilde{g} \mathbf{D} \omega\right)$. The corresponding potential $V$ can be strongly singular. 
16.3. The Schrödinger equation. The operators (16.14) and (16.1) (with $g=\omega^{2} \widetilde{g}$ ) satisfy the identity $\mathcal{H}=\omega^{-1} \widehat{\mathcal{A}} \omega^{-1}$. Let $g^{0}$ be the effective matrix for the operator (16.1). Now the function $Q(\mathbf{x})=\left(f(\mathbf{x}) f(\mathbf{x})^{*}\right)^{-1}$ takes the form $Q(\mathbf{x})=\omega^{2}(\mathbf{x})$. Then, by (16.13), we have $\bar{Q}=\overline{\omega^{2}}=1$ and $f_{0}=(\bar{Q})^{-1 / 2}=1$. Now the operator (8.5) takes the form

$$
\mathcal{H}^{0}=\mathbf{D}^{*} g^{0} \mathbf{D} \text {. }
$$

Thus, $\mathcal{H}^{0}$ coincides with the effective operator for the operator $\widehat{\mathcal{A}}=\mathbf{D}^{*} g \mathbf{D}=\mathbf{D}^{*} \widetilde{g} \omega^{2} \mathbf{D}$ (see (16.3)).

Now, we consider the operator

$$
\mathcal{H}_{\varepsilon}=\left(\omega^{\varepsilon}\right)^{-1} \mathbf{D}^{*} g^{\varepsilon} \mathbf{D}\left(\omega^{\varepsilon}\right)^{-1}, \quad g^{\varepsilon}(\mathbf{x})=\left(\omega^{\varepsilon}(\mathbf{x})\right)^{2} \widetilde{g}^{\varepsilon}(\mathbf{x}) .
$$

If condition (16.11) is satisfied, then (16.16) can be rewritten in the initial terms:

$$
\mathcal{H}_{\varepsilon}=\mathbf{D}^{*} \widetilde{g}^{\varepsilon} \mathbf{D}+\varepsilon^{-2} V^{\varepsilon} .
$$

It is important that the expression (16.17) contains a large factor $\varepsilon^{-2}$ at the rapidly oscillating potential $V^{\varepsilon}$.

We consider the Cauchy problem of the form (14.9) for the Schrödinger equation

$$
i \frac{\partial u_{\varepsilon}(\mathbf{x}, \tau)}{\partial \tau}=\mathcal{H}_{\varepsilon} u_{\varepsilon}(\mathbf{x}, \tau), \quad\left(\omega^{\varepsilon}(\mathbf{x})\right)^{-1} u_{\varepsilon}(\mathbf{x}, 0)=\phi(\mathbf{x}),
$$

where $\phi \in L_{2}\left(\mathbb{R}^{d}\right)$ is a given function.

Let $u_{0}(\mathbf{x}, \tau)$ be the solution of the "homogenized" Cauchy problem (see (14.10))

$$
i \frac{\partial u_{0}(\mathbf{x}, \tau)}{\partial \tau}=\mathcal{H}^{0} u_{0}(\mathbf{x}, \tau), \quad u_{0}(\mathbf{x}, 0)=\phi(\mathbf{x}) .
$$

Note that, by (16.15), problem (16.18) coincides with (16.8). Theorem 14.3 is applicable. Under the assumption $\phi \in H^{s}\left(\mathbb{R}^{d}\right), 0<s \leq 3$, as $\varepsilon \rightarrow 0$ the product $\left(\omega^{\varepsilon}\right)^{-1} u_{\varepsilon}$ tends to $u_{0}$ in the $L_{2}\left(\mathbb{R}^{d}\right)$-norm for a fixed $\tau \in \mathbb{R}$. We have

$$
\left\|\left(\omega^{\varepsilon}\right)^{-1} u_{\varepsilon}(\cdot, \tau)-u_{0}(\cdot, \tau)\right\|_{L_{2}\left(\mathbb{R}^{d}\right)} \leq \varepsilon^{s / 3} \mathcal{C}_{s}(\tau)\|\phi\|_{H^{s}\left(\mathbb{R}^{d}\right)} .
$$

The constant $\mathcal{C}_{s}(\tau)$ is of order $O\left(|\tau|^{s / 3}\right)$ (for large values of $|\tau|$ ) and depends on the norms $\|\widetilde{g}\|_{L_{\infty}},\left\|\widetilde{g}^{-1}\right\|_{L_{\infty}},\|\omega\|_{L_{\infty}},\left\|\omega^{-1}\right\|_{L_{\infty}}$, and on the parameters of the lattice $\Gamma$. Under condition (16.11), this constant is controlled in terms of $\|\widetilde{g}\|_{L_{\infty}},\left\|\widetilde{g}^{-1}\right\|_{L_{\infty}},\|V\|_{L_{q}(\Omega)}$, and the parameters of the lattice.

For large values of time $|\tau|=\varepsilon^{-\alpha}$, we have

$$
\left\|\left(\omega^{\varepsilon}\right)^{-1} u_{\varepsilon}\left(\cdot, \pm \varepsilon^{-\alpha}\right)-u_{0}\left(\cdot, \pm \varepsilon^{-\alpha}\right)\right\|_{L_{2}\left(\mathbb{R}^{d}\right)} \leq \varepsilon^{s(1-\alpha) / 3} \mathcal{C}_{s}(1)\|\phi\|_{H^{s}\left(\mathbb{R}^{d}\right)}, \quad 0<\alpha<1 .
$$

If $\phi \in L_{2}\left(\mathbb{R}^{d}\right)$, then as $\varepsilon \rightarrow 0$ the product $\left(\omega^{\varepsilon}\right)^{-1} u_{\varepsilon}$ tends to $u_{0}$ strongly in $L_{2}\left(\mathbb{R}^{d}\right)$ for $\tau \in \mathbb{R}$ fixed. The difference $\left(\omega^{\varepsilon}\right)^{-1} u_{\varepsilon}\left(\cdot, \pm \varepsilon^{-\alpha}\right)-u_{0}\left(\cdot, \pm \varepsilon^{-\alpha}\right)$ tends to zero strongly in $L_{2}\left(\mathbb{R}^{d}\right)$, provided $0<\alpha<1$.

Also, the nonhomogeneous problem of the form (14.15) with the operator (16.16) can be considered. Theorem 14.5 may be applied to this problem.

Remark 16.2. Consider the magnetic Schrödinger operator

$$
\mathcal{M}=(\mathbf{D}-\mathbf{A}(\mathbf{x}))^{*} \widetilde{g}(\mathbf{x})(\mathbf{D}-\mathbf{A}(\mathbf{x}))+V(\mathbf{x})
$$

with periodic metric $\widetilde{g}(\mathbf{x})$, magnetic potential $\mathbf{A}(\mathbf{x})$, and electric potential $V(\mathbf{x})$. If the magnetic potential is sufficiently small, and the bottom of the spectrum of $\mathcal{M}$ is the point $\lambda_{0}=0$, then $\mathcal{M}$ admits a factorization of the form (4.6). This factorization was found in $\underline{\mathrm{Sh}}$. (See also BSu4, Subsection 11.3].) In this case, Theorems 14.3 and 14.5 apply to the corresponding Cauchy problems with the operator

$$
\mathcal{M}_{\varepsilon}=\left(\mathbf{D}-\varepsilon^{-1} \mathbf{A}^{\varepsilon}(\mathbf{x})\right)^{*} \widetilde{g}^{\varepsilon}(\mathbf{x})\left(\mathbf{D}-\varepsilon^{-1} \mathbf{A}^{\varepsilon}(\mathbf{x})\right)+\varepsilon^{-2} V^{\varepsilon}(\mathbf{x}) .
$$




\section{$\S 17$. The TWo-Dimensional Pauli EQUation}

17.1. Model examples. The examples considered below will be useful for the study of the two-dimensional Pauli operator in Subsections 17.3-17.5. However, these examples are of interest in themselves. The corresponding operators were considered in BSu2, Chapter 5, Subsection 1.3].

Let $d=2, n=m=1$, and let $\omega_{ \pm}(\mathbf{x})$ be a $\Gamma$-periodic function such that

$$
\omega_{ \pm}(\mathbf{x})>0, \quad \omega_{ \pm}, \omega_{ \pm}^{-1} \in L_{\infty} .
$$

Consider the operator

$$
\mathcal{B}_{ \pm}=\partial_{ \pm} \omega_{ \pm}^{2}(\mathbf{x}) \partial_{\mp}
$$

where $\partial_{ \pm}:=D_{1} \pm i D_{2}$. The operators $\mathcal{B}_{+}$and $\mathcal{B}_{-}$are of the same type, and it is convenient to consider them in parallel. The operator $\mathcal{B}_{ \pm}$is of the form (6.1) with $g=\omega_{ \pm}^{2}, b(\mathbf{D})=D_{1} \mp i D_{2}$. Since $m=n=1$, the effective constant $g_{ \pm}^{0}$ is given by the formula

$$
g_{ \pm}^{0}=\underline{\left(\omega_{ \pm}^{2}\right)}=\left(|\Omega|^{-1} \int_{\Omega} \omega_{ \pm}(\mathbf{x})^{-2} d \mathbf{x}\right)^{-1} .
$$

In accordance with (6.8), the effective operator $\mathcal{B}_{ \pm}^{0}$ for $\mathcal{B}_{ \pm}$is given by

$$
\mathcal{B}_{ \pm}^{0}=\partial_{ \pm} g_{ \pm}^{0} \partial_{\mp}=-g_{ \pm}^{0} \Delta .
$$

Now we consider the matrix version of the preceding example. Let $d=n=m=2$. Let $\omega_{ \pm}(\mathbf{x})$ be two (scalar) $\Gamma$-periodic functions satisfying condition (17.1). In $L_{2}\left(\mathbb{R}^{2} ; \mathbb{C}^{2}\right)$, we consider the operator

$$
\mathcal{B}_{\times}=\operatorname{diag}\left(\partial_{-} \omega_{-}^{2} \partial_{+}, \partial_{+} \omega_{+}^{2} \partial_{-}\right)=: \operatorname{diag}\left\{\mathcal{B}_{-}, \mathcal{B}_{+}\right\} .
$$

The operator (17.3) admits a factorization of the form (6.1):

$$
\mathcal{B}_{\times}=b_{\times}(\mathbf{D}) g_{\times}(\mathbf{x}) b_{\times}(\mathbf{D}),
$$

where

$$
b_{\times}(\mathbf{D})=\left(\begin{array}{cc}
0 & \partial_{-} \\
\partial_{+} & 0
\end{array}\right), \quad g_{\times}=\operatorname{diag}\left\{\omega_{+}^{2}, \omega_{-}^{2}\right\} .
$$

Since $m=n=2$, the effective matrix for the operator (17.3) is given by

$$
g_{\times}^{0}=\underline{g_{\times}}=\operatorname{diag}\left\{g_{+}^{0}, g_{-}^{0}\right\}, \quad g_{ \pm}^{0}=\underline{\left(\omega_{ \pm}^{2}\right)} .
$$

The effective operator $\mathcal{B}_{\times}^{0}$ is given by

$$
\mathcal{B}_{\times}^{0}=b_{\times}(\mathbf{D}) g_{\times}^{0} b_{\times}(\mathbf{D})=\operatorname{diag}\left\{\mathcal{B}_{-}^{0}, \mathcal{B}_{+}^{0}\right\}, \quad \mathcal{B}_{ \pm}^{0}=-g_{ \pm}^{0} \Delta .
$$

17.2. Homogenization problems for model operators. Now we introduce the operators

$$
\mathcal{B}_{ \pm, \varepsilon}=\partial_{ \pm}\left(\omega_{ \pm}^{\varepsilon}\right)^{2} \partial_{\mp}, \quad \mathcal{B}_{\times, \varepsilon}=b_{\times}(\mathbf{D}) g_{\times}^{\varepsilon}(\mathbf{x}) b_{\times}(\mathbf{D})=\operatorname{diag}\left\{\mathcal{B}_{-, \varepsilon}, \mathcal{B}_{+, \varepsilon}\right\}
$$

The coefficients of these operators oscillate rapidly as $\varepsilon \rightarrow 0$.

Consider a pair of Cauchy problems for the scalar functions $v_{ \pm, \varepsilon}(\mathbf{x}, \tau)$ :

$$
\begin{array}{ll}
i \frac{\partial v_{+, \varepsilon}}{\partial \tau}=\partial_{+}\left(\omega_{+}^{\varepsilon}(\mathbf{x})\right)^{2} \partial_{-} v_{+, \varepsilon}, & v_{+, \varepsilon}(\mathbf{x}, 0)=\phi_{+}(\mathbf{x}), \\
i \frac{\partial v_{-, \varepsilon}}{\partial \tau}=\partial_{-}\left(\omega_{-}^{\varepsilon}(\mathbf{x})\right)^{2} \partial_{+} v_{-, \varepsilon}, & v_{-, \varepsilon}(\mathbf{x}, 0)=\phi_{-}(\mathbf{x}),
\end{array}
$$


where $\phi_{ \pm} \in L_{2}\left(\mathbb{R}^{2}\right)$ are given functions. Theorem 14.1 is applicable to these problems. The corresponding homogenized problems take the form

$$
i \frac{\partial v_{ \pm, 0}}{\partial \tau}=-g_{ \pm}^{0} \Delta v_{ \pm, 0}, \quad v_{ \pm, 0}(\mathbf{x}, 0)=\phi_{ \pm}(\mathbf{x}),
$$

where the constants $g_{ \pm}^{0}$ are defined by (17.5).

By Theorem 14.1, if $\phi_{ \pm} \in H^{s}\left(\mathbb{R}^{2}\right), 0<s \leq 3$, then as $\varepsilon \rightarrow 0$ the function $v_{ \pm, \varepsilon}$ tends to $v_{ \pm, 0}$ in $L_{2}\left(\mathbb{R}^{2}\right)$ for $\tau \in \mathbb{R}$ fixed. We have

$(17.10 \pm) \quad\left\|v_{ \pm, \varepsilon}(\cdot, \tau)-v_{ \pm, 0}(\cdot, \tau)\right\|_{L_{2}\left(\mathbb{R}^{2}\right)} \leq \varepsilon^{s / 3} \widehat{\mathcal{C}}_{ \pm, s}(\tau)\left\|\phi_{ \pm}\right\|_{H^{s}\left(\mathbb{R}^{2}\right)}, \quad \tau \in \mathbb{R}, \quad 0<\varepsilon \leq 1$.

The constant $\widehat{\mathcal{C}}_{ \pm, s}(\tau)$ is of order $O\left(|\tau|^{s / 3}\right.$ ) (for large $|\tau|$ ) and depends on the norms $\left\|\omega_{ \pm}\right\|_{L_{\infty}},\left\|\omega_{ \pm}^{-1}\right\|_{L_{\infty}}$ and on the parameters of the lattice. Also, the difference $v_{ \pm, \varepsilon}-v_{ \pm, 0}$ satisfies an estimate of the form (14.3) for $|\tau|=\varepsilon^{-\alpha}, 0<\alpha<1$. Statement $2^{\circ}$ of Theorem 14.1 about strong convergence is also true.

Also, nonhomogeneous Cauchy problems of the form (14.4) with the operators (17.7 \pm ) can be considered, and Theorem 14.2 applies to such problems.

Now we consider the Cauchy problem for a vector-valued function $\mathbf{v}_{\times, \varepsilon}(\mathbf{x}, \tau)$ :

$$
i \frac{\partial \mathbf{v}_{\times, \varepsilon}}{\partial \tau}=\mathcal{B}_{\times, \varepsilon} \mathbf{v}_{\times, \varepsilon}, \quad \mathbf{v}_{\times, \varepsilon}(\mathbf{x}, 0)=\phi(\mathbf{x}),
$$

where $\phi \in L_{2}\left(\mathbb{R}^{2} ; \mathbb{C}^{2}\right)$ is a given function. We put $\phi=\operatorname{col}\left\{\phi_{-}, \phi_{+}\right\}$. Clearly, we have $\mathbf{v}_{\times, \varepsilon}=\operatorname{col}\left\{v_{-, \varepsilon}, v_{+, \varepsilon}\right\}$, where the $v_{ \pm, \varepsilon}$ are solutions of problems $(17.8 \pm)$.

Theorem 14.1 is applicable to problem (17.11). The corresponding homogenized problem is of the form

$$
i \frac{\partial \mathbf{v}_{\times, 0}}{\partial \tau}=\mathcal{B}_{\times}^{0} \mathbf{v}_{\times, 0}, \quad \mathbf{v}_{\times, 0}(\mathbf{x}, 0)=\phi(\mathbf{x}),
$$

where $\mathcal{B}_{\times}^{0}$ is the effective operator (17.6). Clearly, we have $\mathbf{v}_{\times, 0}=\operatorname{col}\left\{v_{-, 0}, v_{+, 0}\right\}$, where $v_{ \pm, 0}$ are solutions of $(17.9 \pm)$.

By Theorem 14.1, if $\phi \in H^{s}\left(\mathbb{R}^{2} ; \mathbb{C}^{2}\right), 0<s \leq 3$, then

$$
\left\|\mathbf{v}_{\times, \varepsilon}(\cdot, \tau)-\mathbf{v}_{\times, 0}(\cdot, \tau)\right\|_{L_{2}\left(\mathbb{R}^{2}\right)} \leq \varepsilon^{s / 3} \widehat{\mathcal{C}}_{\times, s}(\tau)\|\phi\|_{H^{s}\left(\mathbb{R}^{2}\right)}, \quad \tau \in \mathbb{R}, \quad 0<\varepsilon \leq 1 .
$$

The constant $\widehat{\mathcal{C}}_{\times, s}(\tau)$ is $O\left(|\tau|^{s / 3}\right)$ (for large $|\tau|$ ) and depends on the norms $\left\|\omega_{+}\right\|_{L_{\infty}}$, $\left\|\omega_{+}^{-1}\right\|_{L_{\infty}},\left\|\omega_{-}\right\|_{L_{\infty}},\left\|\omega_{-}^{-1}\right\|_{L_{\infty}}$ and on the parameters of the lattice. Of course, estimate (17.12) could be derived from the pair of estimates (17.10 \pm ) and from the decomposition of problem (17.11) in two scalar problems (17.8 \pm ). The difference $\mathbf{v}_{\times, \varepsilon}-\mathbf{v}_{\times, 0}$ satisfies an estimate of the form (14.3) with $|\tau|=\varepsilon^{-\alpha}, 0<\alpha<1$. Statement $2^{\circ}$ of Theorem 14.1 is also true.

17.3. Definition and factorization of the two-dimensional Pauli operator. (See [BSu2, Chapter 6, Subsection 2.1].) Suppose that the magnetic potential $\mathbf{A}=\left\{A_{1}, A_{2}\right\}$ is a $\Gamma$-periodic real vector-valued function on $\mathbb{R}^{2}$ such that

$$
\mathbf{A} \in L_{\rho}\left(\Omega ; \mathbb{C}^{2}\right), \quad \rho>2 .
$$

We recall the standard notation for the Pauli matrices

$$
\sigma_{1}=\left(\begin{array}{cc}
0 & 1 \\
1 & 0
\end{array}\right), \quad \sigma_{2}=\left(\begin{array}{cc}
0 & -i \\
i & 0
\end{array}\right), \quad \sigma_{3}=\left(\begin{array}{cc}
1 & 0 \\
0 & -1
\end{array}\right) .
$$

In $L_{2}\left(\mathbb{R}^{2} ; \mathbb{C}^{2}\right)$, we consider the operator

$$
\mathcal{D}:=\left(D_{1}-A_{1}\right) \sigma_{1}+\left(D_{2}-A_{2}\right) \sigma_{2}, \quad \operatorname{Dom} \mathcal{D}=H^{1}\left(\mathbb{R}^{2} ; \mathbb{C}^{2}\right) .
$$

By definition, the Pauli operator $\mathcal{P}$ is the square of the operator $\mathcal{D}$ :

$$
\mathcal{P}:=\mathcal{D}^{2}=\left(\begin{array}{cc}
P_{-} & 0 \\
0 & P_{+}
\end{array}\right) \text {. }
$$


The precise definition of the operator $\mathcal{P}$ is given in terms of the quadratic form

$$
\|\mathcal{D} \mathbf{u}\|_{L_{2}\left(\mathbb{R}^{2}\right)}^{2}, \quad \mathbf{u} \in H^{1}\left(\mathbb{R}^{2} ; \mathbb{C}^{2}\right),
$$

which is closed in $L_{2}\left(\mathbb{R}^{2} ; \mathbb{C}^{2}\right)$. If the potential $\mathbf{A}$ is sufficiently smooth, then the blocks $P_{ \pm}$of the operator (17.15) are given by

$$
P_{ \pm}=(\mathbf{D}-\mathbf{A}(\mathbf{x}))^{2} \pm B(\mathbf{x}), \quad B:=\partial_{1} A_{2}-\partial_{2} A_{1} .
$$

The expression $B(\mathbf{x})$ corresponds to the strength of the magnetic field.

We use a well-known factorization for the operators (17.14) and (17.15). A gauge transformation allows us to assume that the potential $\mathbf{A}$ is subject to the conditions

$$
\operatorname{div} \mathbf{A}=0, \quad \int_{\Omega} \mathbf{A} d \mathbf{x}=0,
$$

and still satisfies (17.13). Under conditions (17.13) and (17.17), there exists a (unique) real-valued $\Gamma$-periodic function $\varphi$ such that

$$
\nabla \varphi=\left\{A_{2},-A_{1}\right\}, \quad \int_{\Omega} \varphi(\mathbf{x}) d \mathbf{x}=0 .
$$

From (17.13) and (17.18) it follows that

$$
\varphi \in \widetilde{W}_{\rho}^{1}(\Omega) \subset \operatorname{Lip} \sigma, \quad \sigma=1-2 \rho^{-1} .
$$

Here $\widetilde{W}_{\rho}^{1}(\Omega)$ is the subspace of the Sobolev class $W_{\rho}^{1}(\Omega)$ that consists of functions whose $\Gamma$-periodic extension belongs to $W_{\rho, \text { loc }}^{1}\left(\mathbb{R}^{2}\right)$.

We put

$$
\omega_{ \pm}(\mathbf{x}):=e^{ \pm \varphi(\mathbf{x})}
$$

and use the notation (17.4) (with $\omega_{ \pm}$as in (17.20)) and also the notation

$$
f_{\times}:=g_{\times}^{1 / 2}=\operatorname{diag}\left\{\omega_{+}, \omega_{-}\right\} .
$$

By (17.19), we have $\omega_{ \pm} \in \widetilde{W}_{\rho}^{1}(\Omega)$ and

$$
\omega_{-}(\mathbf{x}) \omega_{+}(\mathbf{x})=1, \quad \mathbf{x} \in \mathbb{R}^{2} .
$$

The operators (17.14) and (17.15) can be written as follows:

$$
\begin{aligned}
& \mathcal{D}=f_{\times}(\mathbf{x}) b_{\times}(\mathbf{D}) f_{\times}(\mathbf{x}), \\
& \mathcal{P}=f_{\times}(\mathbf{x}) b_{\times}(\mathbf{D}) g_{\times}(\mathbf{x}) b_{\times}(\mathbf{D}) f_{\times}(\mathbf{x}) .
\end{aligned}
$$

The blocks $P_{ \pm}$of the operator (17.24) look like this:

$$
P_{+}=\omega_{-} \partial_{+} \omega_{+}^{2} \partial_{-} \omega_{-}, \quad P_{-}=\omega_{+} \partial_{-} \omega_{-}^{2} \partial_{+} \omega_{+} .
$$

Thus, $P_{+}=Y^{*} Y, P_{-}=Y Y^{*}$, where $Y:=\omega_{+} \partial_{-} \omega_{-}$. It is convenient to view the expressions (17.23)-(17.25) as the definition of the operators $\mathcal{D}, \mathcal{P}$, and $P_{ \pm}$, assuming that $\omega_{ \pm}$are arbitrary $\Gamma$-periodic functions satisfying (17.1) and also (17.22). More precisely, the operator $\mathcal{D}$ is given by (17.23) on the domain

$$
\operatorname{Dom} \mathcal{D}=\left\{\mathbf{u} \in L_{2}\left(\mathbb{R}^{2} ; \mathbb{C}^{2}\right): f_{\times} \mathbf{u} \in H^{1}\left(\mathbb{R}^{2} ; \mathbb{C}^{2}\right)\right\} .
$$

The operator $\mathcal{P}$ is defined via the quadratic form $\|\mathcal{D} \mathbf{u}\|_{L_{2}\left(\mathbb{R}^{2}\right)}^{2}, \mathbf{u} \in \operatorname{Dom} \mathcal{D}$. The blocks $P_{ \pm}$are defined via the quadratic forms

$$
\left\|\omega_{ \pm} \partial_{\mp} \omega_{\mp} u\right\|_{L_{2}\left(\mathbb{R}^{2}\right)}^{2}, \quad \omega_{\mp} u \in H^{1}\left(\mathbb{R}^{2}\right) .
$$

At the same time, the expression for the magnetic field strength in (17.16) has no direct meaning. 
Clearly, $\operatorname{Ker} Y=\operatorname{Ker} Y^{*}=\{0\}$. Therefore, the operators $P_{+}=Y^{*} Y$ and $P_{-}=Y Y^{*}$ are unitarily equivalent. Obviously, the study of the operator $\mathcal{P}$ reduces to that of the blocks $P_{ \pm}$.

17.4. The effective characteristics for the two-dimensional Pauli operator. The operators $P_{ \pm}$and $\mathcal{P}$ are of the form (4.6). We start with the operators $P_{ \pm}$. Now $d=2$ and $m=n=1$. Formulas (17.25) give representations of the operators $P_{ \pm}$in the form $\mathcal{A}(g, f)$ (see (4.6)) with $b(\mathbf{D})=D_{1} \mp i D_{2}, g=\omega_{ \pm}^{2}$, and $f=\omega_{\mp}$. Herewith,

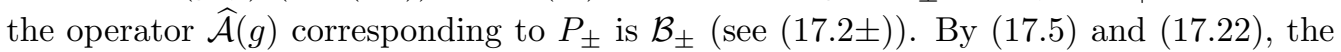
effective constants $g_{ \pm}^{0}$ are defined as follows:

$$
g_{ \pm}^{0}=\left(|\Omega|^{-1} \int_{\Omega} \omega_{\mp}^{2} d \mathbf{x}\right)^{-1}=: \omega_{ \pm, 0}^{2} .
$$

The role of $Q(\mathbf{x})$ (see (8.1)) for the operator $P_{ \pm}$is played by the function $Q_{ \pm}=\omega_{ \pm}^{2}$. Then, by $\left(17.26 \pm\right.$ ), we have $\overline{Q_{ \pm}}=\overline{\left(\omega_{ \pm}^{2}\right)}=\left(g_{\mp}^{0}\right)^{-1}=\left(\omega_{\mp, 0}\right)^{-2}$. The role of $f_{0}$ (see (8.2)) for $P_{ \pm}$is played by the constant $\left(\overline{Q_{ \pm}}\right)^{-1 / 2}=\left(g_{\mp}^{0}\right)^{1 / 2}=\omega_{\mp, 0}$. Accordingly, the role of the operator $\mathcal{A}^{0}$ (see (8.5)) for $P_{+}$is played by $P_{+}^{0}=\omega_{-, 0} \partial_{+} g_{+}^{0} \partial_{-} \omega_{-, 0}=-\gamma \Delta$, and for $P_{-}$the role of $\mathcal{A}^{0}$ is played by $P_{-}^{0}=\omega_{+, 0} \partial_{-} g_{-}^{0} \partial_{+} \omega_{+, 0}=-\gamma \Delta$. Here

$$
\gamma:=g_{+}^{0} g_{-}^{0}=|\Omega|^{2}\left\|\omega_{+}\right\|_{L_{2}(\Omega)}^{-2}\left\|\omega_{-}\right\|_{L_{2}(\Omega)}^{-2} .
$$

Thus,

$$
P_{+}^{0}=P_{-}^{0}=-\gamma \Delta .
$$

Now we briefly describe the operator $\mathcal{P}$. Here $m=n=d=2$. Formula (17.24) gives a representation of the operator $\mathcal{P}$ in the form $\mathcal{A}(g, f)$ (see (4.6)), where $b(\mathbf{D})=b_{\times}(\mathbf{D})$, $g=g_{\times}$, and $f=f_{\times}$are defined by (17.4) and (17.21). The role of the corresponding operator $\widehat{\mathcal{A}}(g)$ is played by the operator $\mathcal{B}_{\times}$defined by (17.3). The effective matrix $g_{\times}^{0}$ is given by (17.5), and the effective operator $\mathcal{B}_{\times}^{0}$ is defined by (17.6). The role of the matrix $Q(\mathbf{x})$ (see (8.1)) for the operator $\mathcal{P}$ is played by the matrix

$$
Q_{\times}=f_{\times}^{-2}=g_{\times}^{-1}=\operatorname{diag}\left\{\omega_{-}^{2}, \omega_{+}^{2}\right\} .
$$

Then, by (17.26 \pm ), we have

$$
\overline{Q_{\times}}=\operatorname{diag}\left\{\left(g_{+}^{0}\right)^{-1},\left(g_{-}^{0}\right)^{-1}\right\} .
$$

The role of $f_{0}$ (see (8.2)) for $\mathcal{P}$ is played by the matrix

$$
f_{\times, 0}=\left(\overline{Q_{\times}}\right)^{-1 / 2}=\operatorname{diag}\left\{\omega_{+, 0}, \omega_{-, 0}\right\} .
$$

Accordingly, the role of the operator $\mathcal{A}^{0}$ (see (8.5)) for $\mathcal{P}$ is played by the operator

$$
\mathcal{P}^{0}=f_{\times, 0} \mathcal{B}_{\times}^{0} f_{\times, 0}=\left(\begin{array}{cc}
-\gamma \Delta & 0 \\
0 & -\gamma \Delta
\end{array}\right),
$$

where $\gamma$ is the number (17.27).

17.5. Homogenization of the Cauchy problem for the two-dimensional Pauli equation. Now we introduce the operators

$$
\begin{aligned}
P_{+, \varepsilon} & =\omega_{-}^{\varepsilon} \partial_{+}\left(\omega_{+}^{\varepsilon}\right)^{2} \partial_{-} \omega_{-}^{\varepsilon}, \quad P_{-, \varepsilon}=\omega_{+}^{\varepsilon} \partial_{-}\left(\omega_{-}^{\varepsilon}\right)^{2} \partial_{+} \omega_{+}^{\varepsilon}, \\
\mathcal{P}_{\varepsilon} & =f_{\times}^{\varepsilon}(\mathbf{x}) b_{\times}(\mathbf{D}) g_{\times}^{\varepsilon}(\mathbf{x}) b_{\times}(\mathbf{D}) f_{\times}^{\varepsilon}(\mathbf{x})=\left(\begin{array}{cc}
P_{-, \varepsilon} & 0 \\
0 & P_{+, \varepsilon}
\end{array}\right),
\end{aligned}
$$

whose coefficients oscillate rapidly for small $\varepsilon$. 
If the potential $\mathbf{A}$ is sufficiently smooth, the operators (17.31) can be rewritten in the form

$$
P_{ \pm, \varepsilon}=\left(\mathbf{D}-\varepsilon^{-1} \mathbf{A}^{\varepsilon}\right)^{2} \pm \varepsilon^{-2} B^{\varepsilon} .
$$

Consider the following Cauchy problems for the scalar-valued functions $u_{ \pm, \varepsilon}(\mathbf{x}, \tau)$ :

$$
i \frac{\partial u_{ \pm, \varepsilon}}{\partial \tau}=P_{ \pm, \varepsilon} u_{ \pm, \varepsilon}, \quad \omega_{\mp}^{\varepsilon}(\mathbf{x}) u_{ \pm, \varepsilon}(\mathbf{x}, 0)=\phi_{ \pm}(\mathbf{x}),
$$

where $\phi_{ \pm} \in L_{2}\left(\mathbb{R}^{2}\right)$ are given functions. Theorem 14.3 is applicable to problems (17.33 \pm ). The corresponding homogenized problems are of the form (see (17.28))

$$
i \frac{\partial u_{ \pm, 0}}{\partial \tau}=-\gamma \Delta u_{ \pm, 0}, \quad \omega_{\mp, 0} u_{ \pm, 0}(\mathbf{x}, 0)=\phi_{ \pm}(\mathbf{x}),
$$

where $\gamma$ is the number (17.27), and the numbers $\omega_{ \pm, 0}$ are defined by $(17.26 \pm)$.

By Theorem 14.3, if $\phi_{ \pm} \in H^{s}\left(\mathbb{R}^{2}\right), 0<s \leq 3$, then as $\varepsilon \rightarrow 0$ the product $\omega_{\mp}^{\varepsilon} u_{ \pm, \varepsilon}$ tends to $\omega_{\mp, 0} u_{ \pm, 0}$ in $L_{2}\left(\mathbb{R}^{2}\right)$ for a fixed $\tau \in \mathbb{R}$. We have

$$
\begin{array}{r}
\left\|\omega_{\mp}^{\varepsilon} u_{ \pm, \varepsilon}(\cdot, \tau)-\omega_{\mp, 0} u_{ \pm, 0}(\cdot, \tau)\right\|_{L_{2}\left(\mathbb{R}^{2}\right)} \leq \varepsilon^{s / 3} \mathcal{C}_{ \pm, s}(\tau)\left\|\phi_{ \pm}\right\|_{H^{s}\left(\mathbb{R}^{2}\right)}, \\
\tau \in \mathbb{R}, \quad 0<\varepsilon \leq 1 .
\end{array}
$$

The constants $\mathcal{C}_{ \pm, s}(\tau)$ are of order $O\left(|\tau|^{s / 3}\right.$ ) (for large $|\tau|$ ) and depend on the norms $\left\|\omega_{+}\right\|_{L_{\infty}},\left\|\omega_{-}\right\|_{L_{\infty}}$ and on the parameters of the lattice. Under condition (17.13), these constants are controlled in terms of the norm $\|\mathbf{A}\|_{L_{\rho}(\Omega)}$ and the parameters of the lattice. The difference $\omega_{\mp}^{\varepsilon} u_{ \pm, \varepsilon}-\omega_{\mp, 0} u_{ \pm, 0}$ satisfies an estimate of the form (14.11) for $|\tau|=\varepsilon^{-\alpha}$, $0<\alpha<1$. The statements about strong convergence (statement $2^{\circ}$ of Theorem 14.3) are true.

Also, nonhomogeneous Cauchy problems of the form (14.15) with operators (17.31) can be considered. Theorem 14.5 applies to such problems.

Finally, we consider the Cauchy problem for the Pauli equation

$$
i \frac{\partial \mathbf{u}_{\varepsilon}}{\partial \tau}=\mathcal{P}_{\varepsilon} \mathbf{u}_{\varepsilon}, \quad f_{\times}^{\varepsilon}(\mathbf{x}) \mathbf{u}_{\varepsilon}(\mathbf{x}, 0)=\phi(\mathbf{x}),
$$

where $\phi \in L_{2}\left(\mathbb{R}^{2} ; \mathbb{C}^{2}\right)$ is a given function. Here the operator $\mathcal{P}_{\varepsilon}$ is defined by (17.32), and $f_{\times}^{\varepsilon}=\operatorname{diag}\left\{\omega_{+}^{\varepsilon}, \omega_{-}^{\varepsilon}\right\}$. Let $\phi=\operatorname{col}\left\{\phi_{-}, \phi_{+}\right\}$. Clearly, $\mathbf{u}_{\varepsilon}=\operatorname{col}\left\{u_{-, \varepsilon}, u_{+, \varepsilon}\right\}$, where

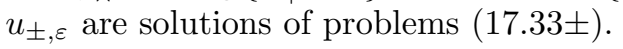

We can apply Theorem 14.3 to problem (17.36). The corresponding homogenized problem is of the form (see (17.30))

$$
i \frac{\partial \mathbf{u}_{0}}{\partial \tau}=-\gamma \Delta \mathbf{u}_{0}, \quad f_{\times, 0} \mathbf{u}_{0}(\mathbf{x}, 0)=\phi(\mathbf{x}),
$$

where $\gamma$ is as in (17.27), and the matrix $f_{\times, 0}$ is defined by (17.29). Clearly, $\mathbf{u}_{0}=$ $\operatorname{col}\left\{u_{-, 0}, u_{+, 0}\right\}$, where $u_{ \pm, 0}$ are solutions of $(17.34 \pm)$.

By Theorem 14.3, if $\phi \in H^{s}\left(\mathbb{R}^{2} ; \mathbb{C}^{2}\right), 0<s \leq 3$, then

$$
\left\|f_{\times}^{\varepsilon} \mathbf{u}_{\varepsilon}(\cdot, \tau)-f_{\times, 0} \mathbf{u}_{0}(\cdot, \tau)\right\|_{L_{2}\left(\mathbb{R}^{2}\right)} \leq \varepsilon^{s / 3} \mathcal{C}_{\times, s}(\tau)\|\phi\|_{H^{s}\left(\mathbb{R}^{2}\right)}, \quad \tau \in \mathbb{R}, \quad 0<\varepsilon \leq 1 .
$$

The constant $\mathcal{C}_{\times, s}(\tau)$ is $O\left(|\tau|^{s / 3}\right.$ ) (for large $|\tau|$ ) and depends on the norms $\left\|\omega_{+}\right\|_{L_{\infty}}$, $\left\|\omega_{-}\right\|_{L_{\infty}}$ and the parameters of the lattice. Obviously, estimate (17.37) could be derived from the pair of estimates (17.35 \pm ) and from the decomposition of problem (17.36) in two scalar problems (17.33土). The difference $f_{\times}^{\varepsilon} \mathbf{u}_{\varepsilon}-f_{\times, 0} \mathbf{u}_{0}$ satisfies an estimate of the form (14.11) for $|\tau|=\varepsilon^{-\alpha}, 0<\alpha<1$. Statement $2^{\circ}$ of Theorem 14.3 is also true.

Theorem 14.5 can be applied to the Cauchy problem of the form (14.15) for the Pauli equation with nontrivial right-hand side. 


\section{§18. Hyperbolic Problems. The acoustics EQUATIOn And The System OF ELASTICITY THEORY}

18.1. The acoustics equation. In $L_{2}\left(\mathbb{R}^{d}\right)$, we consider the operator $\widehat{\mathcal{A}}=\mathbf{D}^{*} g(\mathbf{x}) \mathbf{D}$ (see (16.1)) with periodic matrix $g(\mathbf{x})$ satisfying condition (16.2). The operator $\widehat{\mathcal{A}}$ describes a periodic acoustical medium. The effective matrix $g^{0}$ is defined in accordance with (16.4) and (16.5), and the effective operator is given by $\widehat{\mathcal{A}}^{0}=\mathbf{D}^{*} g^{0} \mathbf{D}$. Let $Q(\mathbf{x})$ be a $\Gamma$-periodic function on $\mathbb{R}^{d}$ such that

$$
Q(\mathbf{x})>0, \quad Q, Q^{-1} \in L_{\infty}
$$

The function $Q(\mathbf{x})$ describes the density of the medium. Consider the operator $\widehat{\mathcal{A}}_{\varepsilon}=$ $\mathbf{D}^{*} g^{\varepsilon}(\mathbf{x}) \mathbf{D}$, whose coefficients oscillate rapidly for small $\varepsilon$.

We consider the Cauchy problem for the acoustics equation in the medium with rapidly oscillating characteristics:

$$
\begin{aligned}
Q^{\varepsilon}(\mathbf{x}) \frac{\partial^{2} z_{\varepsilon}(\mathbf{x}, \tau)}{\partial \tau^{2}} & =-\widehat{\mathcal{A}}_{\varepsilon} z_{\varepsilon}(\mathbf{x}, \tau), \quad \mathbf{x} \in \mathbb{R}^{d}, \quad \tau \in \mathbb{R} \\
z_{\varepsilon}(\mathbf{x}, 0) & =\phi(\mathbf{x}), \quad \frac{\partial z_{\varepsilon}}{\partial \tau}(\mathbf{x}, 0)=\psi(\mathbf{x})
\end{aligned}
$$

where $\phi, \psi \in L_{2}\left(\mathbb{R}^{d}\right)$ are given functions.

Problem (18.1) is of the form (15.10). Therefore, Theorem 15.4 is applicable. The homogenized problem (see (15.11)) takes the form

$$
\bar{Q} \frac{\partial^{2} z_{0}(\mathbf{x}, \tau)}{\partial \tau^{2}}=-\widehat{\mathcal{A}}^{0} z_{0}(\mathbf{x}, \tau), \quad z_{0}(\mathbf{x}, 0)=\phi(\mathbf{x}), \quad \frac{\partial z_{0}}{\partial \tau}(\mathbf{x}, 0)=\psi(\mathbf{x}) .
$$

By Theorem 15.4 , if $\phi, \psi \in H^{s}\left(\mathbb{R}^{d}\right), 0<s \leq 2$, then as $\varepsilon \rightarrow 0$ the solution $z_{\varepsilon}$ tends to $z_{0}$ in $L_{2}\left(\mathbb{R}^{d}\right)$ for $\tau \in \mathbb{R}$ fixed. We have

$$
\begin{array}{r}
\left\|z_{\varepsilon}(\cdot, \tau)-z_{0}(\cdot, \tau)\right\|_{L_{2}\left(\mathbb{R}^{d}\right)} \leq \varepsilon^{s / 2}\left(\mathfrak{C}_{s}(\tau)\|\phi\|_{H^{s}\left(\mathbb{R}^{d}\right)}+\mathfrak{C}_{s}^{\prime}(\tau)\|\psi\|_{H^{s}\left(\mathbb{R}^{d}\right)}\right), \\
\tau \in \mathbb{R}, \quad 0<\varepsilon \leq 1 .
\end{array}
$$

Here $\mathfrak{C}_{s}(\tau)$ is $O\left(|\tau|^{s / 2}\right)$ and $\mathfrak{C}_{s}^{\prime}(\tau)$ is $O\left(|\tau|^{1+s / 2}\right)$ for large values of $|\tau| ; \mathfrak{C}_{s}(\tau)$ and $\mathfrak{C}_{s}^{\prime}(\tau)$ depend on the norms $\|g\|_{L_{\infty}},\left\|g^{-1}\right\|_{L_{\infty}},\|Q\|_{L_{\infty}},\left\|Q^{-1}\right\|_{L_{\infty}}$, and on the parameters of the lattice $\Gamma$. For $\tau= \pm \varepsilon^{-\alpha}$ we have

$$
\begin{array}{r}
\left\|z_{\varepsilon}\left(\cdot, \pm \varepsilon^{-\alpha}\right)-z_{0}\left(\cdot, \pm \varepsilon^{-\alpha}\right)\right\|_{L_{2}\left(\mathbb{R}^{d}\right)} \leq \varepsilon^{s(1-\alpha) / 2}\left(\mathfrak{C}_{s}(1)\|\phi\|_{H^{s}\left(\mathbb{R}^{d}\right)}+\varepsilon^{-\alpha} \mathfrak{C}_{s}^{\prime}(1)\|\psi\|_{H^{s}\left(\mathbb{R}^{2}\right)}\right) \\
0<\varepsilon \leq 1 .
\end{array}
$$

Here $0<\alpha<s(s+2)^{-1}$ for $\psi \neq 0$, and $0<\alpha<1$ for $\psi=0$.

If it is only known that $\phi, \psi \in L_{2}\left(\mathbb{R}^{d}\right)$, then as $\varepsilon \rightarrow 0$ the solution $z_{\varepsilon}$ tends to $z_{0}$ strongly in $L_{2}\left(\mathbb{R}^{d}\right)$ for $\tau \in \mathbb{R}$ fixed. If $\phi \in L_{2}\left(\mathbb{R}^{d}\right)$ and $\psi=0$, then the difference $z_{\varepsilon}\left(\cdot, \pm \varepsilon^{-\alpha}\right)-z_{0}\left(\cdot, \pm \varepsilon^{-\alpha}\right)$ tends to zero strongly in $L_{2}\left(\mathbb{R}^{d}\right)$ for $0<\alpha<1$.

For the operator $\widehat{\mathcal{A}}_{\varepsilon}=\mathbf{D}^{*} g^{\varepsilon}(\mathbf{x}) \mathbf{D}$, we can also consider a problem of the form (15.14) with nontrivial right-hand side in the equation. Theorem 15.6 is applicable to this problem.

18.2. The operator of elasticity theory. Let $d \geq 2$. We represent the operator of elasticity theory in the form used in [BSu2, Chapter $5, \S 2]$. Let $\zeta$ be an orthogonal second rank tensor in $\mathbb{R}^{d}$; in the standard orthonormal basis in $\mathbb{R}^{d}$, it can be represented by a matrix $\zeta=\left\{\zeta_{j l}\right\}_{j, l=1}^{d}$. We shall consider symmetric tensors $\zeta$, which will be identified with vectors $\zeta_{*} \in \mathbb{C}^{m}, 2 m=d(d+1)$, by the following rule. The vector $\zeta_{*}$ is formed by all components $\zeta_{j l}, j \leq l$, and the pairs $(j, l)$ are put in order in some fixed way. 
For the displacement vector $\mathbf{u} \in H^{1}\left(\mathbb{R}^{d} ; \mathbb{C}^{d}\right)$, we introduce the deformation tensor

$$
e(\mathbf{u})=\frac{1}{2}\left\{\frac{\partial u_{j}}{\partial x_{l}}+\frac{\partial u_{l}}{\partial x_{j}}\right\} .
$$

Let $e_{*}(\mathbf{u})$ be the vector corresponding to the tensor $e(\mathbf{u})$ in accordance with the rule described above. The relation

$$
b(\mathbf{D}) \mathbf{u}=-i e_{*}(\mathbf{u})
$$

determines an $(m \times d)$-matrix homogeneous DO $b(\mathbf{D})$ uniquely; the symbol of this DO is a matrix with real entries. For instance, with an appropriate ordering, we have

$$
b(\boldsymbol{\xi})=\left(\begin{array}{cc}
\xi^{1} & 0 \\
\frac{1}{2} \xi^{2} & \frac{1}{2} \xi^{1} \\
0 & \xi^{2}
\end{array}\right), \quad d=2, \quad b(\boldsymbol{\xi})=\left(\begin{array}{ccc}
\xi^{1} & 0 & 0 \\
\frac{1}{2} \xi^{2} & \frac{1}{2} \xi^{1} & 0 \\
0 & \xi^{2} & 0 \\
0 & \frac{1}{2} \xi^{3} & \frac{1}{2} \xi^{2} \\
0 & 0 & \xi^{3} \\
\frac{1}{2} \xi^{3} & 0 & \frac{1}{2} \xi^{1}
\end{array}\right), \quad d=3 .
$$

Let $\sigma(\mathbf{u})$ be the stress tensor, and let $\sigma_{*}(\mathbf{u})$ be the corresponding vector. In the adopted way of writing, the Hooke law about proportionality of stresses and deformations can be expressed by the relation

$$
\sigma_{*}(\mathbf{u}):=g(\mathbf{x}) e_{*}(\mathbf{u})
$$

where $g(\mathbf{x})$ is an $(m \times m)$-matrix (which gives a "concise" description of the Hooke tensor). The matrix $g(\mathbf{x})$ characterizes the parameters of the elastic (in general, anisotropic) medium under study. We assume that the matrix-valued function $g(\mathbf{x})$ is $\Gamma$-periodic and that $g(\mathbf{x})>0, g, g^{-1} \in L_{\infty}$.

The energy of elastic deformations is given by the quadratic form

$$
\begin{array}{r}
w[\mathbf{u}, \mathbf{u}]=\frac{1}{2} \int_{\mathbb{R}^{d}}\left\langle\sigma_{*}(\mathbf{u}), e_{*}(\mathbf{u})\right\rangle_{\mathbb{C}^{m}} d \mathbf{x}=\frac{1}{2} \int_{\mathbb{R}^{d}}\langle g(\mathbf{x}) b(\mathbf{D}) \mathbf{u}, b(\mathbf{D}) \mathbf{u}\rangle_{\mathbb{C}^{m}} d \mathbf{x} \\
\mathbf{u} \in H^{1}\left(\mathbb{R}^{d} ; \mathbb{C}^{d}\right) .
\end{array}
$$

The operator $\mathcal{W}$ generated by this form is the operator of elasticity theory. Thus,

$$
2 \mathcal{W}=b(\mathbf{D})^{*} g b(\mathbf{D})=\widehat{\mathcal{A}}(g)
$$

Now $n=d$ and $m=d(d+1) / 2$.

In the case of an isotropic medium, the matrix $g(\mathbf{x})$ depends only on two functional Lamé parameters $\lambda(\mathbf{x})$ and $\mu(\mathbf{x})$. The parameter $\mu$ is the shear modulus. Often, another parameter $K(\mathbf{x})$ is introduced instead of $\lambda(\mathbf{x}) ; K(\mathbf{x})$ is called the modulus of volume compression. We shall need yet another modulus $\beta(\mathbf{x})$. Here are the relations:

$$
K(\mathbf{x})=\lambda(\mathbf{x})+\frac{2 \mu(\mathbf{x})}{d}, \quad \beta(\mathbf{x})=\mu(\mathbf{x})+\frac{\lambda(\mathbf{x})}{2}
$$

The modulus $\lambda(\mathbf{x})$ may be negative. In the isotropic case, the conditions that ensure the positive definiteness of the matrix $g(\mathbf{x})$ are as follows: $\mu(\mathbf{x}) \geq \mu_{0}>0, K(\mathbf{x}) \geq K_{0}>0$. 
As an example, we write out the matrix $g$ in the isotropic case for $d=2,3$ :

$$
\begin{aligned}
& g_{\mu, K}(\mathbf{x})=\left(\begin{array}{ccc}
K+\mu & 0 & K-\mu \\
0 & 4 \mu & 0 \\
K-\mu & 0 & K+\mu
\end{array}\right), \quad d=2, \\
& g_{\mu, K}(\mathbf{x})=\frac{1}{3}\left(\begin{array}{cccccc}
3 K+4 \mu & 0 & 3 K-2 \mu & 0 & 3 K-2 \mu & 0 \\
0 & 12 \mu & 0 & 0 & 0 & 0 \\
3 K-2 \mu & 0 & 3 K+4 \mu & 0 & 3 K-2 \mu & 0 \\
0 & 0 & 0 & 12 \mu & 0 & 0 \\
3 K-2 \mu & 0 & 3 K-2 \mu & 0 & 3 K+4 \mu & 0 \\
0 & 0 & 0 & 0 & 0 & 12 \mu
\end{array}\right), \quad d=3 .
\end{aligned}
$$

18.3. Homogenization of the Cauchy problem for the elasticity equations. We consider the operator $\mathcal{W}_{\varepsilon}=\frac{1}{2} \widehat{\mathcal{A}}\left(g^{\varepsilon}\right)$ with rapidly oscillating coefficients. The effective matrix $g^{0}$ and the effective operator $\mathcal{W}^{0}=\frac{1}{2} \widehat{\mathcal{A}}^{0}$ are constructed in accordance with the general rules (see (6.5), (6.6), and (6.8)).

Let $Q(\mathbf{x})$ be a $\Gamma$-periodic $(d \times d)$-matrix such that

$$
Q(\mathbf{x})>0, \quad Q, Q^{-1} \in L_{\infty} .
$$

Usually, $Q(\mathbf{x})$ is a scalar-valued function describing the density of the medium. We assume that $Q(\mathbf{x})$ is a matrix-valued function, in order to take possible anisotropy into account. Let $Q^{\varepsilon}(\mathbf{x})=Q\left(\varepsilon^{-1} \mathbf{x}\right)$.

We consider the following Cauchy problem for the system of elasticity theory:

$$
Q^{\varepsilon}(\mathbf{x}) \frac{\partial^{2} \mathbf{u}_{\varepsilon}(\mathbf{x}, \tau)}{\partial \tau^{2}}=-\mathcal{W}_{\varepsilon} \mathbf{u}_{\varepsilon}(\mathbf{x}, \tau), \quad \mathbf{u}_{\varepsilon}(\mathbf{x}, 0)=\phi(\mathbf{x}), \quad \frac{\partial \mathbf{u}_{\varepsilon}}{\partial \tau}(\mathbf{x}, 0)=\boldsymbol{\psi}(\mathbf{x}),
$$

where $\boldsymbol{\phi}, \boldsymbol{\psi} \in L_{2}\left(\mathbb{R}^{d} ; \mathbb{C}^{d}\right)$ are given functions.

Problem (18.3) is of the form (15.10). Theorem 15.4 is applicable to this problem. The homogenized problem takes the form

$$
\bar{Q} \frac{\partial^{2} \mathbf{u}_{0}(\mathbf{x}, \tau)}{\partial \tau^{2}}=-\mathcal{W}^{0} \mathbf{u}_{0}(\mathbf{x}, \tau), \quad \mathbf{u}_{0}(\mathbf{x}, 0)=\phi(\mathbf{x}), \quad \frac{\partial \mathbf{u}_{0}}{\partial \tau}(\mathbf{x}, 0)=\boldsymbol{\psi}(\mathbf{x}) .
$$

By Theorem 15.4 , if $\phi, \boldsymbol{\psi} \in H^{s}\left(\mathbb{R}^{d} ; \mathbb{C}^{d}\right), 0<s \leq 2$, then as $\varepsilon \rightarrow 0$ the solution $\mathbf{u}_{\varepsilon}$ tends to $\mathbf{u}_{0}$ in $L_{2}\left(\mathbb{R}^{d} ; \mathbb{C}^{d}\right)$ for $\tau \in \mathbb{R}$ fixed. We have

$$
\begin{array}{r}
\left\|\mathbf{u}_{\varepsilon}(\cdot, \tau)-\mathbf{u}_{0}(\cdot, \tau)\right\|_{L_{2}\left(\mathbb{R}^{d}\right)} \leq \varepsilon^{s / 2}\left(\mathfrak{C}_{s}(\tau)\|\boldsymbol{\phi}\|_{H^{s}\left(\mathbb{R}^{d}\right)}+\mathfrak{C}_{s}^{\prime}(\tau)\|\boldsymbol{\psi}\|_{H^{s}\left(\mathbb{R}^{d}\right)}\right), \\
\tau \in \mathbb{R}, \quad 0<\varepsilon \leq 1 .
\end{array}
$$

Here $\mathfrak{C}_{s}(\tau)$ is $O\left(|\tau|^{s / 2}\right)$, and $\mathfrak{C}_{s}^{\prime}(\tau)$ is $O\left(|\tau|^{1+s / 2}\right)$ for large $|\tau| ; \mathfrak{C}_{s}(\tau)$ and $\mathfrak{C}_{s}^{\prime}(\tau)$ depend on the norms $\|g\|_{L_{\infty}},\left\|g^{-1}\right\|_{L_{\infty}},\|Q\|_{L_{\infty}},\left\|Q^{-1}\right\|_{L_{\infty}}$, and on the parameters of the lattice $\Gamma$. For $\tau= \pm \varepsilon^{-\alpha}$, we have

$$
\begin{aligned}
& \left\|\mathbf{u}_{\varepsilon}\left(\cdot, \pm \varepsilon^{-\alpha}\right)-\mathbf{u}_{0}\left(\cdot, \pm \varepsilon^{-\alpha}\right)\right\|_{L_{2}\left(\mathbb{R}^{d}\right)} \leq \varepsilon^{s(1-\alpha) / 2}\left(\mathfrak{C}_{s}(1)\|\boldsymbol{\phi}\|_{H^{s}\left(\mathbb{R}^{d}\right)}+\varepsilon^{-\alpha} \mathfrak{C}_{s}^{\prime}(1)\|\boldsymbol{\psi}\|_{H^{s}\left(\mathbb{R}^{d}\right)}\right), \\
& 0<\varepsilon \leq 1 \text {. }
\end{aligned}
$$

Here $0<\alpha<s(s+2)^{-1}$ for $\boldsymbol{\psi} \neq 0$, and $0<\alpha<1$ for $\boldsymbol{\psi}=0$.

If it is only known that $\boldsymbol{\phi}, \boldsymbol{\psi} \in L_{2}\left(\mathbb{R}^{d} ; \mathbb{C}^{d}\right)$, then as $\varepsilon \rightarrow 0$ the solution $\mathbf{u}_{\varepsilon}$ tends to $\mathbf{u}_{0}$ strongly in $L_{2}\left(\mathbb{R}^{d} ; \mathbb{C}^{d}\right)$ for $\tau \in \mathbb{R}$ fixed. If $\phi \in L_{2}\left(\mathbb{R}^{d} ; \mathbb{C}^{d}\right)$ and $\boldsymbol{\psi}=0$, then the difference $\mathbf{u}_{\varepsilon}\left(\cdot, \pm \varepsilon^{-\alpha}\right)-\mathbf{u}_{0}\left(\cdot, \pm \varepsilon^{-\alpha}\right)$ tends to zero strongly in $L_{2}\left(\mathbb{R}^{d} ; \mathbb{C}^{d}\right)$ for $0<\alpha<1$.

For the operator $\mathcal{W}_{\varepsilon}$, we can also consider a problem of the form (15.14) with nontrivial right-hand side in the equation. Theorem 15.6 is applicable to this problem.

18.4. The Hill body. In mechanics (see, e.g., [ZhKO]), an elastic isotropic medium with constant shear modulus $\mu(\mathbf{x})=\mu_{0}=$ const is called a Hill body. In this case, a 
simpler factorization for the operator $\mathcal{W}$ is possible (see BSu2, Chapter 5, Subsection $2.3]$ ). Now the energy form (18.2) can be written as

$$
w[\mathbf{u}, \mathbf{u}]=\int_{\mathbb{R}^{d}}\left\langle g_{\wedge} b_{\wedge}(\mathbf{D}) \mathbf{u}, b_{\wedge}(\mathbf{D}) \mathbf{u}\right\rangle_{\mathbb{C}^{m \wedge}} d \mathbf{x} .
$$

Here $m_{\wedge}=1+d(d-1) / 2$. The $\left(m_{\wedge} \times d\right)$-matrix $b_{\wedge}(\boldsymbol{\xi})$ can be described as follows. The first row in $b_{\wedge}(\boldsymbol{\xi})$ is $\left(\xi^{1}, \xi^{2}, \ldots, \xi^{d}\right)$. The other rows correspond to (different) pairs of indices $(j, l), 1 \leq j<l \leq d$. The element in the $(j, l)$ th row and the $j$ th column is $\xi^{l}$, and the element in the $(j, l)$ th row and the $l$ th column is $\left(-\xi^{j}\right)$; all other elements of the $(j, l)$ th row are zero. The order of the rows is irrelevant. Finally,

$$
g_{\wedge}(\mathbf{x})=\operatorname{diag}\left\{\beta(\mathbf{x}), \mu_{0} / 2, \mu_{0} / 2, \ldots, \mu_{0} / 2\right\} .
$$

Thus, by (18.5), we have

$$
\mathcal{W}=b_{\wedge}(\mathbf{D})^{*} g_{\wedge}(\mathbf{x}) b_{\wedge}(\mathbf{D}) .
$$

In [BSu2, Chapter 5, Subsection 2.3], it was shown that the effective matrix $g_{\wedge}^{0}$ coincides with $\underline{g_{\wedge}}$ :

$$
g_{\wedge}^{0}=\underline{g_{\wedge}}=\operatorname{diag}\left\{\underline{\beta}, \mu_{0} / 2, \mu_{0} / 2, \ldots, \mu_{0} / 2\right\} .
$$

The effective operator can be written as

$$
\mathcal{W}^{0}=b_{\wedge}(\mathbf{D})^{*} g_{\wedge}^{0} b_{\wedge}(\mathbf{D}) .
$$

For the chosen factorization of the operator $\mathcal{W}_{\varepsilon}=b_{\wedge}(\mathbf{D})^{*} g_{\wedge}^{\varepsilon}(\mathbf{x}) b_{\wedge}(\mathbf{D})$, the Cauchy problem (18.3) remains the same. In the homogenized problem (18.4), the effective operator $\mathcal{W}^{0}$ is defined by (18.7) and is calculated explicitly, due to relation (18.6). In the case of the Hill body, the solutions of problem (18.3) satisfy all the statements formulated in Subsection 18.3.

\section{§19. APPENDIX}

19.1. In conclusion, we demonstrate a trick that gives simple proofs of some weaker versions of Theorems 12.1 and 12.2. We start with the following abstract lemma.

Lemma 19.1. Let $B_{0}$ and $B_{1}$ be selfadjoint nonnegative operators in a Hilbert space $\mathcal{H}$. Let $G_{j}:=\left(B_{j}+I\right)^{-1}, j=0,1$, and $L:=G_{1}-G_{0}$. Then

$$
\left\|\left(e^{-i B_{1} \tau}-e^{-i B_{0} \tau}\right) G_{0}^{2}\right\|_{\mathcal{H} \rightarrow \mathcal{H}} \leq(|\tau|+2)\|L\|_{\mathcal{H} \rightarrow \mathcal{H}}, \quad \tau \in \mathbb{R} .
$$

Proof. We denote $\mathcal{E}(\tau):=e^{-i B_{1} \tau} G_{1} G_{0} e^{i B_{0} \tau}$. Then

$$
\begin{aligned}
\frac{d \mathcal{E}(\tau)}{d \tau} & =i e^{-i B_{1} \tau}\left(-B_{1} G_{1} G_{0}+G_{1} G_{0} B_{0}\right) e^{i B_{0} \tau} \\
& =i e^{-i B_{1} \tau}\left(-\left(B_{1}+I\right) G_{1} G_{0}+G_{1} G_{0}\left(B_{0}+I\right)\right) e^{i B_{0} \tau}=i e^{-i B_{1} \tau} L e^{i B_{0} \tau} .
\end{aligned}
$$

Hence,

$$
\mathcal{E}(\tau)-\mathcal{E}(0)=e^{-i B_{1} \tau} G_{1} G_{0} e^{i B_{0} \tau}-G_{1} G_{0}=i \int_{0}^{\tau} e^{-i B_{1} \rho} L e^{i B_{0} \rho} d \rho .
$$

We multiply (19.2) by $e^{-i B_{0} \tau}$ from the right:

$$
e^{-i B_{1} \tau} G_{1} G_{0}-G_{1} G_{0} e^{-i B_{0} \tau}=i \int_{0}^{\tau} e^{-i B_{1} \rho} L e^{i B_{0}(\rho-\tau)} d \rho .
$$

We have $G_{1} G_{0}=\left(G_{0}+L\right) G_{0}=G_{0}^{2}+L G_{0}$. Then the left-hand side of (19.3) can be transformed to

$$
e^{-i B_{1} \tau} G_{1} G_{0}-G_{1} G_{0} e^{-i B_{0} \tau}=\left(e^{-i B_{1} \tau}-e^{-i B_{0} \tau}\right) G_{0}^{2}+e^{-i B_{1} \tau} L G_{0}-L G_{0} e^{-i B_{0} \tau}
$$


Relations (19.3) and (19.4) imply the identity

$$
\left(e^{-i B_{1} \tau}-e^{-i B_{0} \tau}\right) G_{0}^{2}=-e^{-i B_{1} \tau} L G_{0}+L G_{0} e^{-i B_{0} \tau}+i \int_{0}^{\tau} e^{-i B_{1} \rho} L e^{i B_{0}(\rho-\tau)} d \rho,
$$

which yields estimate (19.1).

19.2. Now we apply Lemma 19.1 with $\mathcal{H}=L_{2}\left(\mathbb{R}^{d} ; \mathbb{C}^{n}\right), B_{1}=\widehat{\mathcal{A}}_{\varepsilon}$, and $B_{0}=\widehat{\mathcal{A}}^{0}$. Here $\widehat{\mathcal{A}}_{\varepsilon}$ is the operator (11.1), and $\widehat{\mathcal{A}}^{0}$ is the corresponding effective operator (6.8). Then

$$
\begin{aligned}
\|\left(\exp \left(-i \widehat{\mathcal{A}}_{\varepsilon} \tau\right)\right. & \left.-\exp \left(-i \widehat{\mathcal{A}}^{0} \tau\right)\right)\left(\widehat{\mathcal{A}}^{0}+I\right)^{-2} \|_{L_{2}\left(\mathbb{R}^{d}\right) \rightarrow L_{2}\left(\mathbb{R}^{d}\right)} \\
& \leq(|\tau|+2)\left\|\left(\widehat{\mathcal{A}}_{\varepsilon}+I\right)^{-1}-\left(\widehat{\mathcal{A}}^{0}+I\right)^{-1}\right\|_{L_{2}\left(\mathbb{R}^{d}\right) \rightarrow L_{2}\left(\mathbb{R}^{d}\right)}
\end{aligned}
$$

Applying estimate (0.5) for the difference of resolvents, we obtain the following statement.

Proposition 19.2. Let $\widehat{\mathcal{A}}_{\varepsilon}$ be the operator (11.1) and let $\widehat{\mathcal{A}}^{0}$ be the corresponding effective operator (6.8). Then

$$
\begin{aligned}
& \left\|\left(\exp \left(-i \widehat{\mathcal{A}}_{\varepsilon} \tau\right)-\exp \left(-i \widehat{\mathcal{A}}^{0} \tau\right)\right)\left(\widehat{\mathcal{A}}^{0}+I\right)^{-2}\right\|_{L_{2}\left(\mathbb{R}^{d}\right) \rightarrow L_{2}\left(\mathbb{R}^{d}\right)} \leq C(|\tau|+2) \varepsilon, \\
& \tau \in \mathbb{R}, 0<\varepsilon \leq 1 .
\end{aligned}
$$

In the same way as Theorem 12.2 was deduced from Theorem 12.1, relation (19.5) implies the following statement.

Proposition 19.3. Under the conditions of Proposition 19.2, for $0 \leq s \leq 4$ we have

$$
\begin{array}{r}
\left\|\exp \left(-i \widehat{\mathcal{A}}_{\varepsilon} \tau\right)-\exp \left(-i \widehat{\mathcal{A}}^{0} \tau\right)\right\|_{H^{s}\left(\mathbb{R}^{d}\right) \rightarrow L_{2}\left(\mathbb{R}^{d}\right)} \leq C_{s}(|\tau|+2)^{s / 4} \varepsilon^{s / 4}, \\
\tau \in \mathbb{R}, \quad 0<\varepsilon \leq 1 .
\end{array}
$$

Though we have succeeded in deducing Proposition 19.3 directly from the "elliptic" estimate (0.5), the result is weaker than the result of Theorem 12.2. For instance, in Theorem 12.2, an estimate of order of $\varepsilon$ is proved for $s=3$, while here such an estimate is proved only for $s=4$.

\section{REFERENCES}

[BaPa] N. S. Bakhvalov and G. P. Panasenko, Homogenization: Averaging processes in periodic media, Nauka, Moscow, 1984; English transl., Math. Appl. (Soviet Ser.), vol. 36, Kluwer Acad. Publ. Group, Dordrecht, 1989. MR0797571 (86m:73049) MR1112788(92d:73002)

[BeLP] A. Bensoussan, J.-L. Lions, and G. Papanicolaou, Asymptotic analysis for periodic structures, Stud. Math. Appl., vol. 5, North-Holland Publ. Co., Amsterdam-New York, 1978, 700 pp. MR0503330 (82h:35001)

[BS] M. Sh. Birman and M. Z. Solomyak, Estimates for the difference of fractional powers of selfadjoint operators under unbounded perturbations, Zap. Nauchn. Sem. Leningrad. Otdel. Mat. Inst. Steklov. (LOMI) 178 (1989), 120-145; English transl., J. Soviet Math. 61 (1992), no. 2, 2018-2035. MR.1037767 (91d:47006)

[BSu1] M. Birman and T. Suslina, Threshold effects near the lower edge of the spectrum for periodic differential operators of mathematical physics, Systems, Approximation, Singular Integral Operators, and Related Topics (Bordeaux, 2000), Oper. Theory Adv. Appl., vol. 129, Birkhäuser, Basel, 2001, pp. 71-107. MR.1882692 (2003f:35220)

[BSu2] - Second order periodic differential operators. Threshold properties and homogenization, Algebra i Analiz 15 (2003), no. 5, 1-108; English transl., St. Petersburg Math. J. 15 (2004), no. 5, 639-714. MR.2068790(2005k:47097)

[BSu3] - Threshold approximations with corrector for the resolvent of a factorized selfadjoint operator family, Algebra i Analiz 17 (2005), no. 5, 69-90; English transl., St. Petersburg Math. J. 17 (2006), no. 5, 745-762. MR2241423 (2008d:47047)

[BSu4] Homogenization with corrector term for periodic elliptic differential operators, Algebra i Analiz 17 (2005), no. 6, 1-104; English transl., St. Petersburg Math. J. 17 (2006), no. 6, 897-973. MR2202045 (2006k:35011) 
[BSu5] - Homogenization with corrector for periodic differential operators. Approximation of solutions in the Sobolev class $H^{1}\left(\mathbb{R}^{d}\right)$, Algebra i Analiz 18 (2006), no. 6, 1-130; English transl., St. Petersburg Math. J. 18 (2007), no. 6, 857-955. MR2307356 (2008d:35008)

[BSu6] - Homogenization of a stationary periodic Maxwell system in the case of constant magnetic permeability, Funktsional. Anal. i Prilozhen. 41 (2007), no. 2, 3-23; English transl., Funct. Anal. Appl. 41 (2007), no. 2, 81-98. MR2345036 (2008i:35014)

[V] E. S. Vasilevskaya, Homogenization with corrector for parabolic Cauchy problem with periodic coefficients, Algebra i Analiz 21 (2009), no. 1, 3-60; English transl. in St. Petersburg Math. J. 21 (2010), no. 1.

[Zh] V. V. Zhikov, On some estimates from homogenization theory, Dokl. Ros. Akad. Nauk 406 (2006), no. 5, 597-601; English transl., Dokl. Math. 73 (2006), 96-99. MR2347318 (2008d:35018)

[ZhKO] V. V. Zhikov, S. M. Kozlov, and O. A. Oleŭnik, Homogenization of differential operators, Nauka, Moscow, 1993; English transl., Springer-Verlag, Berlin, 1994. MR1318242 (96h:35003a) MR:1329546 (96h:35003b)

[ZhPas1] V. V. Zhikov and S. E. Pastukhova, On operator estimates for some problems in homogenization theory, Russ. J. Math. Phys. 12 (2005), no. 4, 515-524. (English) MR2201316 (2007c:35014)

[ZhPas2] — Estimates of homogenization for a parabolic equation with periodic coefficients, Russ. J. Math. Phys. 13 (2006), no. 2, 224-237. MR2262826 (2007k:35025)

[Ka] T. Kato, Perturbation theory for linear operators, 2nd ed., Grundlehren Math. Wiss., Bd. 132, Springer-Verlag, Berlin-New York, 1976. MR0407617 (53:11389)

[Pas] S. E. Pastukhova, On some estimates in homogenization problems of elasticity theory, Dokl. Ros. Akad. Nauk 406 (2006), no. 5, 604-608; English transl., Dokl. Math. 73 (2006), 102-106. MR.2347320 (2008d:35016)

[Sa] E. Sanchez-Palencia, Nonhomogeneous media and vibration theory, Lecture Notes in Phys., vol. 127, Springer-Verlag, Berlin-New York, 1980. MR.0578345 (82j:35010)

[Su1] T. A. Suslina, On the homogenization of periodic parabolic systems, Funktsional. Anal. i Prilozhen. 38 (2004), no. 4, 86-90; English transl., Funct. Anal. Appl. 38 (2004), no. 4, 309-312. MR 2117512 (2005j:35008)

[Su2] Homogenization of a periodic parabolic Cauchy problem, Nonlinear Equations and Spectral Theory, Amer. Math. Soc. Transl. Ser. 2, vol. 220, Amer. Math. Soc., Providence, RI, 2007, pp. 201-233. MR2343612 (2008k:35030)

[Su3] - On homogenization of a periodic Maxwell system, Funktsional. Anal. i Prilozhen. 38 (2004), no. 3, 90-94; English transl., Funct. Anal. Appl. 38 (2004), no. 3, 234-237. MR2095137 (2005g:35017)

[Su4] , Homogenization of a stationary periodic Maxwell system, Algebra i Analiz 16 (2004), no. 5, 162-244; English transl., St. Petersburg Math. J. 16 (2005), no. 5, 863-922. MR2106671 (2005h:35019)

[Su5] Homogenization with corrector for a stationary periodic Maxwell system, Algebra i Analiz 19 (2007), no. 3, 183-235; English transl., St. Petersburg Math. J. 19 (2008), no. 3, 455-494. MR2340710 (2008j:35017)

[Sh] R. G. Shterenberg, On the structure of the lower edge of the spectrum of the periodic magnetic Schrödinger operator with small magnetic potential, Algebra i Analiz 17 (2005), no. 5, 232-243; English transl., St. Petersburg Math. J. 17 (2006), no. 5, 865-873. MR2241429 (2007f:35046)

Department of Physics, St. Petersburg State University, Ul'yanovskaya 3, Petrodvorets, 198504 St. Petersburg, Russia

E-mail address: mbirman@list.ru

Department of Physics, St. Petersburg State University, Ul'yanovskaya 3, Petrodvorets, 198504 St. Petersburg, Russia

E-mail address: suslina@list.ru

Received 10/AUG/2008

Translated by T. A. SUSLINA 